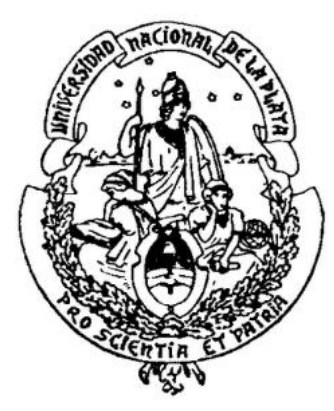

\title{
CONTROL DE FLUJO SOBRE LA ESTELA CERCANA DE PERFILES AERODINÁMICOS MEDIANTE LA IMPLEMENTACIÓN DE MINI-FLAPS GURNEY
}

\author{
Tesis de Doctorado \\ Mauricio E. CAMOCARDI \\ Presentada ante la Facultad de Ingeniería de la \\ Universidad Nacional de La Plata \\ como requisito para la obtención del grado académico de \\ DOCTOR EN INGENIERÍA
}

Dirección de tesis:

Dr. Jorge Colman Lerner
Dr. Julio Marañón Di Leo

Jurado de tesis:

Dr. Jorge Lassig

Dr. Sergio Elaskar

Fecha de defensa oral y pública: 26 de abril de 2012 


\section{Agradecimientos}

Quiero expresar mi agradecimiento a todos los que de alguna manera han colaborado para que esta tesis fuera posible.

A mis directores Jorge y Julio, que me han dado la posibilidad de llevar adelante el doctorado y me han guiado en este largo camino.

A Sebastián y Ana por haber dedicado parte de su tiempo incondicionalmente.

A mis compañeros del laboratorio que han colaborado de alguna u otra forma brindándome un clima de amistad cada día.

Al Profesor Ulfilas Boldes por brindarnos sus valiosos conocimientos y experiencias en mecánica de los fluidos.

Finalmente a mi familia, mi padre y hermanos...

... y a todos aquellos que desde la tierra o el cielo han hecho algo por mí. 


\section{ÍNDICE GENERAL}

Resumen

$\begin{array}{ll}\text { Abreviaturas } & \mathrm{X}\end{array}$

$\begin{array}{ll}\text { Lista de Figuras } & \text { XII }\end{array}$

$\begin{array}{ll}\text { Lista de Tablas } & \text { XV }\end{array}$

1. Introducción 1

1.1. Motivación de la Tesis 1

1.2. Problemáticas Abordadas 2

1.3. Organización y Estructura de la Tesis 5

1.4. Principales Aportes 6

2. Conceptos Generales $\quad 7$

2.1. Aerodinámica de Perfiles $\quad 7$

2.2. Turbulencia 10

2.3. Control de Flujo 13

3. Metodología Experimental y Equipos $\quad 17$

3.1. Introducción 17

3.2. Ensayos en Túnel de Viento 17

3.3. Construcción de Modelos 17

3.4. Sistemas de Actuación $\quad 19$

3.4.1. Mini-flap Gurney Rotante 20

3.4.2. Mini-flap Gurney Rotante (variante $30^{\circ}$ ) 20

3.4.3. Mini-flap Gurney Móvil 21

3.5. Túnel de Viento 23

3.6. Equipos de Medición 24

3.6.1. Anemómetro de Hilo Caliente 24

3.6.2. Posicionador Vertical 27 
4. Procesamiento de Datos 32

4.1. Análisis de las Señales 32

4.2. Análisis Estadístico de Amplitud de Señal 34

4.2.1. Valor Medio 34

4.2.2. Varianza y Desvío Standard 35

4.2.3. Estadística Conjunta de Dos Señales 36

4.3. Análisis en Dominio del Tiempo 37

$\begin{array}{ll}\text { 4.3.1. Autocorrelación } & 37\end{array}$

4.4. Análisis en Dominio de Frecuencia $\quad 39$

4.4.1. Análisis de Fourier 39

5. Análisis de Resultados 45

5.1. Perfil de Velocidades Medias 45

5.1.1. Componente $u \quad 45$

5.1.1.1. Caso Mini-flap Gurney Rotante 45

5.1.1.2. Caso Mini-flap Gurney Rotante (Variante $30^{\circ}$ ) 47

5.1.1.3. Caso Mini-flap Gurney Móvil 49

5.1.1.4. Comparación de Dispositivos - Velocidad Media $u \quad 50$

$\begin{array}{ll}\text { 5.1.2. Componente } v & 50\end{array}$

5.1.2.1. Caso Mini-flap Gurney Rotante $\quad 50$

5.1.2.2. Caso Mini-flap Gurney Rotante (Variante $30^{\circ}$ ) 52

5.1.2.3. Caso Mini-flap Gurney Móvil 53

5.1.2.4. Comparación de Dispositivos - Velocidad Media $v \quad 54$

5.2. Perfil de Intensidad de Turbulencia $\quad 55$

5.2.1. Componente $u \quad 55$

5.2.1.1. Caso Mini-flap Gurney Rotante 55

5.2.1.2. Caso Mini-flap Gurney Rotante (Variante 30) 56

5.2.1.3. Caso Mini-flap Gurney Móvil 58

5.2.1.4. Comparación de Dispositivos - Intensidad de Turbulencia $u \quad 59$

$\begin{array}{ll}5.2 .2 \text {. Componente } v & 60\end{array}$

5.2.2.1. Caso Mini-flap Gurney Rotante $\quad 60$

5.2.2.2. Caso Mini-flap Gurney Rotante (Variante $30^{\circ}$ ) 61

5.2.2.3. Caso Mini-flap Gurney Móvil 62

5.2.2.4. Comparación de Dispositivos - Intensidad de Turbulencia $v \quad 64$

5.3. Vectores de Velocidad Media $\quad 65$

5.3.1. Caso Mini-flap Gurney Rotante 65

5.3.2. Caso Mini-flap Gurney Rotante (Variante $30^{\circ}$ ) 66 
5.4. Escalas Espaciales de Turbulencia

5.5. Espectros de Densidad de Potencia (PSD)

6. Conclusiones 81

6.1. Evaluación de Resultados

6.2. Conclusiones Generales 84

6.3. Futuras Líneas de Investigación 85

A.Características de Perfil NACA $4412 \quad 86$

B. Especificaciones Técnicas de Instrumental 88

$\begin{array}{lr}\text { Bibliografía } & 90\end{array}$ 


\section{RESUMEN}

La curvatura del borde de fuga y su geometría junto con la influencia sobre la condición de Kutta pueden ser usadas para manipular la sustentación, la resistencia de presiones y la estela de un perfil. Algunos investigadores han analizado varios mecanismos de control pasivo y activo de flujo para incrementar el coeficiente de sustentación, en particular, durante el despegue y aterrizaje de una aeronave. Uno de esos mecanismos es el mini-flap Gurney, el cual consiste en una pequeña placa plana localizada en la superficie inferior del perfil, tan cerca como sea posible del borde de fuga, a lo largo de la envergadura.

El objetivo es lograr un desplazamiento del punto de estagnación posterior (condición de Kutta) con un consecuente incremento de circulación y por lo tanto alcanzar una mejora en la sustentación. Uno de los investigadores pioneros en esta área fue Liebeck quien estudió un perfil simétrico tipo Newman con un mini-flap Gurney de altura de $1.25 \%$ c donde $c$ es la cuerda del perfil. Él encontró un importante incremento de sustentación asociado al incremento del downwash en la estela cercana. Por otro lado, investigadores realizaron estudios que corroboraron que como otros dispositivos de control de flujo pasivo en el borde de fuga, el mini-flap Gurney aumenta la sustentación, el máximo coeficiente de sustentación y la pendiente de las curvas de $C_{L}$ versus ángulo de ataque, con pequeños cambios en la resistencia y en el ángulo de pérdida. Además, dichos autores encontraron que la mayor altura de tales dispositivos era la altura local de la capa límite en la superficie inferior cerca del borde de fuga. También observaron que la estela cercana era del tipo de una calle de vórtices de von Kármán.

El desprendimiento de vórtices incrementa la succión en la superficie superior cerca del borde de fuga y, al mismo tiempo, desacelera el flujo en la superficie inferior con el consecuente incremento de presión en ese lado. Estas diferencias de presión incrementan la circulación global alrededor del perfil y, de esta forma, la sustentación. Un mini-flap Gurney en la superficie inferior cerca del borde de fuga retrasará la pérdida, promoviendo crecimientos del máximo coeficiente de sustentación. Por esa razón algunos diseñadores consideraron el uso de dichos mini-flaps combinados con otros dispositivos de control de flujo para lograr dispositivos de hiper-sustentación en aeronaves menos complejos que los clásicos, sin pérdida de performance durante las fases de despegue y aterrizaje. Si observamos la estela, en particular, la estela cercana, podemos concluir que el crecimiento de la circulación está asociado a un incremento del downwash. Esto requiere mayor 
asimetría de la estela cercana. Desde nuestro punto de vista, no es realista la concepción de un modelo clásico del flujo de estela, como una calle simétrica similar a la de von Kármán, porque debido a su simetría no habrá un downwash neto asociado con un incremento de sustentación.

Más aún, en la región de la estela cercana de un perfil con un mini-flap en la superficie inferior, bajo condiciones de sustentación, la intensidad del vórtice desprendido de la superficie superior será diferente que el que se desprende del de la superficie inferior. Esta asimetría será responsable del downwash neto extra y, por lo tanto, del incremento de sustentación. Los experimentos realizados por autores en trabajos previos, mostraron que en la región de la estela cercana, donde el sistema de vórtices comienza su formación, hay un pico importante de frecuencia en el espectro instantáneo de velocidades, generando la llamada inestabilidad de estela.

Existen muchos trabajos relacionados con la implementación de dispositivos de control de flujo en el borde de fuga. Algunos de ellos propusieron el uso de micro-placas capaces de moverse y hacer control de flujo activo sobre rotores de palas de turbinas eólicas. Otros, sugirieron el uso de mini-flaps Gurney activos para disminuir el desarrollo e intensidad de la estela.

La motivación del presente trabajo se centró en el análisis del uso de mini-flaps tipo Gurney como elemento activo de control de flujo con la finalidad de modular las capas de corte que generan un crecimiento de la circulación y subsecuentemente un incremento en la sustentación y/o variaciones en los parámetros que gobiernan el comportamiento de las estructuras turbulentas detrás del borde de fuga. 


\section{Abreviaturas}

$\begin{array}{ll}F G & \text { Flap Gurney } \\ h & \text { Altura del Mini-flap Gurney } \\ \rho & \text { Densidad del aire } \\ c & \text { Cuerda del ala } \\ U_{\infty} & \text { Velocidad de la corriente libre } \\ S & \text { Superficie de referencia } \\ L & \text { Lift: Sustentación } \\ D & \text { Drag: Resistencia aerodinámica } \\ C_{L} & \text { Coeficiente de sustentación } \\ C_{D} & \text { Coeficiente de resistencia aerodinámica } \\ x, y & \text { Ejes de coordenadas longitudinal y vertical } \\ u, v & \text { Componentes longitudinal y vertical de velocidad } \\ R e & \text { Número de Reynolds } \\ S t & \text { Número de Strouhal } \\ t & \text { Tiempo } \\ x(t) & \text { Señal muestreada función del tiempo } \\ \bar{x} & \text { Valor medio de la señal muestreada } \\ x^{\prime}(t) & \text { Fluctuaciones de la señal muestreada función del tiempo } \\ \sigma_{x} & \text { Desvío standard de las fluctuaciones de } x(t) \\ R_{x}(\tau) & \text { Autocorrelación de la función } x(t) \\ R_{x y}(\tau) & \text { Correlación cruzada } \\ C_{x} & \text { Coeficiente de autocorrelación de } x(t) \\ C_{u}, C_{v} & \text { Coeficiente de autocorrelación de las componentes } u \mathrm{y} v \\ T & \text { Tiempo total de muestreo } \\ N & \text { Número de muestras } \\ \Delta t & \text { Fntervalo de muestreo } \\ f_{m} & \text { Frecuencia de muestreo } \\ F F T & \text { Fast Fourier Transform: transformada rápida de Fourier } \\ F & \text { Frecuencia de activación de mecanismos } \\ k & t_{u}, I t_{v}\end{array}$


$T_{I}$

$L_{I}$

$S(n)$

$P S D_{\text {adim }}$

$f$
Escala integral de la turbulencia

Escala espacial de la turbulencia

Función densidad de potencia espectral (PSD)

Densidad de potencia espectral adimensional

Frecuencia de desprendimiento de vórtices 


\section{LISTA DE FIGURAS}

2.1. Clasificación de las estrategias de control de flujo. $\quad 14$

2.2. Control activo de flujo predeterminado. 14

2.3. Control activo reactivo de flujo con alimentación. 15

2.4. Control activo reactivo de flujo con re-alimentación. 15

3.1. Perfil NACA 4412. 18

3.2. Modelo en fase de construcción. 19

3.3. Movimientos de despliegue de mecanismos. 19

3.4. Esquema del mecanismo mini-flap Gurney rotante. 20

3.5. Esquema del mecanismo rotante alternativo. 21

3.6. Esquema del mecanismo del mini-flap Gurney móvil. 22

3.7. Modelo de ala con mini-flap Gurney móvil finalizado. 22

3.8. Sección de prueba del túnel de viento. 23

3.9. Anemómetro DANTEC Stream-Line. 25

3.10. Sensores de velocidad y temperatura. 25

3.11. Conexionado del sistema de anemometría de hilo caliente. 26

3.12. Equipamiento en el túnel de viento. 27

3.13. Vista en planta del modelo montado en el túnel de viento. 29

3.14. Ubicación de los puntos de comparación en el Ala 1.

3.15. Ubicación de los puntos de comparación en el Ala 2 y 3.

4.1. Muestreo discreto de una señal continua. 34

4.2. Función autocorrelación de una medición de velocidad instantánea de viento. $\quad 38$

4.3. Ejemplo de espectro de una señal. 41

4.4. Efecto del muestreo a una frecuencia inferior a la frecuencia de Nyquist. 42

4.5. Ventana rectangular implícita al analizar un intervalo finito $(0, T)$. 43

4.6. Ventana Hanning implícita al analizar un intervalo finito $(0, T)$. 44

5.1. Perfil de velocidades medias "u" al 2\%c - Mini-flap Rotante. 46

5.2. Perfil de velocidades medias "u" al 75\%c - Mini-flap Rotante. 47

5.3. Perfil de velocidades medias "u" al $2 \%$ c - Mini-flap Rotante $\left(30^{\circ}\right)$. 
5.4. Perfil de velocidades medias " $u$ " al $4 \% \mathrm{c}$ - Mini-flap Rotante $\left(30^{\circ}\right)$.

5.5. Perfil de velocidades medias "u" al 10\%c - Mini-flap Rotante $\left(30^{\circ}\right)$.

5.6. Perfil de velocidades medias "u" al 2\%c - Mini-flap Móvil. 49

5.7. Perfil de velocidades medias "u" al 4\%c - Mini-flap Móvil. 49

5.8. Perfil de velocidades medias "u" al 10\%c - Mini-flap Móvil. 50

5.9. Perfil de velocidades medias "v" al 2\%c - Mini-flap Rotante. 51

5.10. Perfil de velocidades medias "v" al $75 \%$ c - Mini-flap Rotante. 51

5.11. Perfil de velocidades medias "v" al $2 \%$ c - Mini-flap Rotante $\left(30^{\circ}\right) . \quad 52$

5.12. Perfil de velocidades medias "v" al $4 \%$ c - Mini-flap Rotante $\left(30^{\circ}\right)$.

5.13. Perfil de velocidades medias "v" al $10 \% \mathrm{c}$ - Mini-flap Rotante $\left(30^{\circ}\right)$.

5.14. Perfil de velocidades medias "v" al 2\%c - Mini-flap Móvil. 53

5.15. Perfil de velocidades medias "v" al 4\%c - Mini-flap Móvil. 54

5.16. Perfil de velocidades medias "v" al 10\%c - Mini-flap Móvil. 54

5.17. Perfil de intensidad de turbulencia "u" al $2 \%$ c Mini-flap Rotante. 56

5.18. Perfil de intensidad de turbulencia "u" al $75 \%$ c - Mini-flap Rotante. 56

5.19. Perfil de intensidad de turbulencia "u" al $2 \%$ c Mini-flap Rotante $\left(30^{\circ}\right)$.

5.20. Perfil de intensidad de turbulencia " $u$ " al $4 \%$ c - Mini-flap Rotante $\left(30^{\circ}\right)$.

5.21. Perfil de intensidad de turbulencia "u" al $10 \%$ c - Mini-flap Rotante $\left(30^{\circ}\right) . \quad 57$

5.22. Perfil de intensidad de turbulencia "u" al 2\%c - Mini-flap Móvil. 58

5.23. Perfil de intensidad de turbulencia "u" al 4\%c - Mini-flap Móvil. 59

5.24. Perfil de intensidad de turbulencia "u" al 10\%c - Mini-flap Móvil. 59

5.25. Perfil de intensidad de turbulencia "v" al 2\%c - Mini-flap Rotante. 60

5.26. Perfil de intensidad de turbulencia "v" al 75\%c - Mini-flap Rotante. 61

5.27. Perfil de intensidad de turbulencia "v" al $2 \%$ c Mini-flap Rotante $\left(30^{\circ}\right)$.

5.28. Perfil de intensidad de turbulencia "v" al 4\%c - Mini-flap Rotante $\left(30^{\circ}\right)$.

5.29. Perfil de intensidad de turbulencia "v" al 10\%c - Mini-flap Rotante $\left(30^{\circ}\right)$.

5.30. Perfil de intensidad de turbulencia "v" al 2\%c - Mini-flap Móvil. 63

5.31. Perfil de intensidad de turbulencia "v" al 4\%c - Mini-flap Móvil. 63

5.32. Perfil de intensidad de turbulencia "v" al 10\%c - Mini-flap Móvil. 63

5.33. Diagrama de vectores de velocidad media - Mini-flap Rotante. 65

5.34. Diagrama de vectores de velocidad media - Mini-flap Rotante $\left(30^{\circ}\right)$. 65

5.35. Diagrama de vectores de velocidad media - Mini-flap Móvil. 66

5.36. Escalas espaciales Mini-flap Rotante (x/c=2\%c, y/c=0\%c). 68

5.37. Escalas espaciales Mini-flap Rotante $(\mathrm{x} / \mathrm{c}=2 \% \mathrm{c}, \mathrm{y} / \mathrm{c}=-2 \% \mathrm{c}) . \quad 69$

5.38. Escalas espaciales Mini-flap Rotante (x/c=75\%c, y/c=0\%c). 69

5.39. Escalas espaciales Mini-flap Rotante (x/c=75\%c, y/c=-2\%c). 70

5.40. Escalas espaciales Mini-flap Rotante $\left(30^{\circ}\right)(\mathrm{x} / \mathrm{c}=2 \% \mathrm{c}, \mathrm{y} / \mathrm{c}=0 \% \mathrm{c}) . \quad 70$

5.41. Escalas espaciales Mini-flap Rotante $\left(30^{\circ}\right)(\mathrm{x} / \mathrm{c}=2 \% \mathrm{c}, \mathrm{y} / \mathrm{c}=-2 \% \mathrm{c})$.

5.42. Escalas espaciales Mini-flap Rotante $\left(30^{\circ}\right)(\mathrm{x} / \mathrm{c}=4 \% \mathrm{c}, \mathrm{y} / \mathrm{c}=0 \% \mathrm{c})$. 
5.43. Escalas espaciales Mini-flap Rotante $\left(30^{\circ}\right)(\mathrm{x} / \mathrm{c}=4 \% \mathrm{c}, \mathrm{y} / \mathrm{c}=-2 \% \mathrm{c})$. 72

5.44. Escalas espaciales Mini-flap Rotante $\left(30^{\circ}\right)(\mathrm{x} / \mathrm{c}=10 \% \mathrm{c}, \mathrm{y} / \mathrm{c}=0 \% \mathrm{c})$.

5.45. Escalas espaciales Mini-flap Rotante $\left(30^{\circ}\right)(\mathrm{x} / \mathrm{c}=10 \% \mathrm{c}, \mathrm{y} / \mathrm{c}=-2 \% \mathrm{c})$.

5.46. Escalas espaciales Mini-flap Móvil (x/c=2\%c, y/c=0\%c). 73

5.47. Escalas espaciales Mini-flap Móvil (x/c=2\%c, y/c=-2\%c). 74

5.48. Escalas espaciales Mini-flap Móvil (x/c=4\%c, y/c=0\%c). 74

5.49. Escalas espaciales Mini-flap Móvil (x/c=4\%c, y/c=-2\%c). 75

5.50. Escalas espaciales Mini-flap Móvil (x/c=10\%c, y/c=0\%c). 75

5.51. Escalas espaciales Mini-flap Móvil (x/c=10\%c, y/c=-2\%c). 76

5.52. Espectro de densidad de potencia Mini-flap Rotante $\mathrm{F} 1$ (x/c=10\%c, y/c=-2\%c). 77 


\section{LISTA DE TABLAS}

$\begin{array}{lll}\text { 5.1. } & \text { Frecuencia reducida para diferentes mecanismos. } & 78\end{array}$

5.2. Strouhal y PSD adimensional vs. k (Mini-flap Rotante, $\mathrm{y} / \mathrm{c}=0 \% \mathrm{c}$ ). 78

5.3. Strouhal y PSD adimensional vs. k (Mini-flap Rotante 30, $\mathrm{y} / \mathrm{c}=0 \% \mathrm{c}$ ). 78

5.4. Strouhal y PSD adimensional vs. $\mathrm{k}$ (Mini-flap Móvil, $\mathrm{y} / \mathrm{c}=0 \% \mathrm{c}$ ). 78

5.5. Strouhal y PSD adimensional vs. k (Mini-flap Rotante, y/c =0\%c). 79

5.6. Strouhal y PSD adimensional vs. k (Mini-flap Rotante 30 , y/c $=0 \% \mathrm{c}$ ). $\quad 79$

5.7. Strouhal y PSD adimensional vs. k (Mini-flap Móvil, y/c = 0\%c). 79

A.1. Coordenadas de perfil NACA $4412 . \quad 86$

A.2. Características geométricas y aerodinámicas del perfil NACA 4412 (Re 500000). 87

B.1. Características técnicas de sensor de velocidad del viento. 88

B.2. Características técnicas de anemómetro de hilo caliente. 89

B.3. Características técnicas de termo-anemómetro de hilo caliente. 89 


\section{CApítulo 1}

\section{INTRODUCCIÓN}

\subsection{Motivación de la Tesis}

Diferentes estudios han demostrado que la estela cercana detrás de un perfil con mini-flap Gurney tiene la forma de una calle de vórtices asimétrica con estructuras vorticosas de diferentes tamaños produciendo fluctuaciones en la circulación global del perfil.

De acuerdo al teorema de Kelvin cada vez que un vórtice se genera y es inyectado en la estela se crea la correspondiente circulación. El desprendimiento periódico de vórtices contra-rotatorios estará vinculado con la aparición de circulaciones de signos opuestos. Este proceso provocará variaciones en el tiempo de la circulación general alrededor del perfil alar produciendo variaciones en el ángulo de ataque instantáneo de la velocidad incidente.

Concentrémonos por un momento en el flujo de estela de cuerpos romos, por ejemplo de un cilindro. Un desprendimiento periódico de vórtices contra-rotantes de igual intensidad producirá variaciones de circulación que generarán variaciones periódicas de ángulo de ataque, de sustentación positiva y negativa y variaciones de resistencia. Un cuerpo que produzca una estela de vórtices de von Kármán experimentará fluctuaciones de este tipo. El promedio de las fuerzas de sustentación resultante será nulo porque esa estela es simétrica y los vórtices iguales y opuestos.

Si ahora nos concentramos en la estela de un perfil, podrá apreciarse que una mayor sustentación está asociada a un mayor downwash. Esto requiere una mayor asimetría del flujo en la estela cercana. Por lo tanto, no resulta realista la concepción de un modelo físico habitual del flujo detrás de un perfil con mini-flap Gurney basado en una estela simétrica de Von Kármán, la cual por ser simétrica es incapaz de provocar una desviación permanente del flujo en una dirección obviamente asociada al incremento permanente de sustentación.

En la estela cercana de un perfil alar equipado con mini-flap Gurney en condiciones de sustentación, los vórtices iniciales que se desprenden desde el extradós deben ser de intensidad diferente a los que se desprenden del intradós. Esta asimetría asegurará la 


\section{CAPÍTULO 1. INTRODUCCIÓN}

desviación media del flujo asociada al incremento de sustentación provocado por estos mini-flaps.

Pero que sucedería si mediante dispositivos de control de flujo logramos aumentar dicha asimetría o anular completamente el vórtice que produce una disminución de la circulación generando estructuras vorticosas de diferentes tamaños detrás del borde de fuga. Esto nos hace pensar que la desviación media se vería incrementada y por ende el downwash en la estela cercana.

Sabemos que desde el mini-flap Gurney fijo se desprenden vórtices asimétricos. Será de especial interés estudiar el campo fluido-dinámico detrás del mini-flap Gurney mediante el análisis de los perfiles de velocidades, las escalas espaciales de las estructuras vorticosas, espectro de densidad de potencia e intensidades de turbulencia, entre otros, cuando se despliegan dispositivos activos en forma alternada entrando y saliendo del modelo o rotando sobre un eje a diferentes frecuencias.

\subsection{Problemáticas Abordadas}

La estela cercana, inmediatamente detrás de un perfil alar con una incidencia tal que genere sustentación es asimétrica. Esta asimetría se debe a las apreciables diferencias entre la capa límite proveniente de la superficie expuesta a baja presión del extradós con respecto a la capa límite de la superficie con sobrepresión del intradós. Al dejar el borde de fuga ambas capas límites se desprenden transformándose en capas de corte que se enrollan en vórtices constituyendo la estructura vorticosa característica de la estela cercana al borde de fuga. A mayor sustentación, las capas límites y las mencionadas capas de corte provenientes del intradós y extradós se diferenciaran aún más, por lo que la estructura vorticosa de la estela cercana será influenciada por la carga aerodinámica y las características del perfil.

Siguiendo corriente abajo al flujo descendente (downwash), se observará inicialmente un aumento de esa velocidad seguido por una rápida disminución de la misma. La asimetría del flujo de estela es grande en la región de apreciable downwash disminuyendo y tendiendo a la simetría a medida que disminuye el downwash en la estela lejana. En un estudio experimental y numérico de la estela cercana de un perfil con sustentación, Hah y Lakshminarayana [1] confirmaron este comportamiento donde la estela alcanza la simetría tan solo a una longitud de cuerda corriente abajo del borde de fuga. Estos autores confirmaron asimismo que la estructura lejana de la estela de un perfil sustentador es prácticamente simétrica no siendo ya influenciada ni por las características aerodinámicas del perfil ni por la carga aerodinámica. El downwash principal ocurre precisamente en la región de estela cercana al borde de fuga distanciada alrededor de una cuerda del mismo.

La ingeniería aeronáutica posee una la larga experiencia en la concepción de la teoría de sustentación clásica debida al sistema vorticoso ligado a un ala. Sin embargo estos 
conocimientos no alcanzan aun para comprender la naturaleza física de los procesos aerodinámicos que provocan el incremento de sustentación en un perfil alar equipado con un mini-flap. Ello se debe a la complejidad introducida por la naturaleza no estacionaria de los vórtices contra-rotantes generados en la estela por ese mini-flap.

Escenarios de flujo no estacionario asociado a desprendimiento de estructuras vorticosas conforman un muy complejo cuadro aerodinámico aún no bien entendido. Al respecto, resulta útil recordar que hasta la fecha para modelos elementales como por ejemplo cilindros circulares no se encontraron teorías capaces de predecir el coeficiente de resistencia en función del número de Reynolds en condiciones de flujo con desprendimiento de vórtices.

Distintos tipos de mini-flaps fueron estudiados por numerosos investigadores [2-9] confirmando un apreciable incremento de sustentación, de la relación sustentaciónresistencia y una reducción de la resistencia de forma para condiciones de gran sustentación con respecto a los mismos valores obtenidos para las mismas alas sin ese mini-flap.

La referencia [6] describe resultados experimentales del comportamiento aerodinámico de estos mini-flaps. Estos autores hipotetizan que los vórtices contrarotantes que se desprenden del borde de fuga inducen líneas de corriente semejantes a las que hubiera provocado una curvada y suave prolongación de la cuerda del perfil aerodinámico introduciendo una curvatura virtual desviando hacia abajo el punto de estagnación posterior virtual libre (condición de Kutta). Los modelos fluido-dinámicos usuales de perfiles con mini-flap describen dicho cambio en la ubicación del punto de estagnación, pero al mismo tiempo asumen la existencia de una estela (simétrica) de calle de vórtices contra-rotatorios de von Kármán sin considerar las mencionadas diferencias de flujo entre la estela cercana y el resto del flujo de estela.

En algunos estudios de eficiencia aerodinámica de perfiles con mini-flaps es frecuente encontrar la hipótesis que presupone que la existencia de la estela de vórtices es siempre perjudicial a una buena relación sustentación-resistencia. A fin de lograr reducciones de resistencia "estabilizando la estela" algunos autores sugieren el uso de ranuras, agujeros, flaps aserrados y cuerpos de estela [10], mientras que otros recomiendan la directa eliminación de la calle de vórtices contra-rotatorios detrás del mini-flap [11]. Por otro lado, hay autores [12] que efectuaron simulaciones numéricas con generadores de vórtices actuando dentro de la capa límite del perfil, cerca del borde de fuga.

En el rango de número de Reynolds de nuestro interés, conocido como bajo número de Reynolds (menores a 106 ), el flujo sobre el extradós para un dado ángulo de ataque en una pequeña zona resulta muy complejo. Si el perfil está pulido, la capa límite permanece laminar hasta que el gradiente de presiones torna de favorable a desfavorable, y en consecuencia, la performance del perfil estará determinada por esta región de flujo laminar que ofrece poca resistencia a la separación. El flujo separado forma una capa de corte libre que es altamente inestable, llegándose a una rápida transición a la turbulencia. 


\section{CAPÍTULO 1. INTRODUCCIÓN}

En esas condiciones el flujo puede llegar a "re-pegarse" debido a la creciente entrada del flujo externo turbulento hacia la región de la pared, con la consecuente energización del flujo re-circulante constituyéndose una burbuja de separación. Las condiciones para que se forme esta burbuja laminar son función del número de Reynolds, la distribución de presiones, la curvatura y rugosidad de la superficie y la turbulencia de la corriente incidente entre otros.

En el rango de bajo número de Reynolds, el flujo separado se orienta según la tangente a la superficie en el punto de separación y la transición a la turbulencia tiene lugar en la capa de corte libre debido a la creciente susceptibilidad de ésta a la transición. La entrada de fluido turbulento de la zona de mayor velocidad provoca la re-adhesión del flujo externo, constituyendo la burbuja laminar ya mencionada. Aguas abajo del punto de re-adherencia, la recientemente formada capa límite turbulenta tiene más energía disponible para luchar contra el gradiente adverso de presiones evitando la separación. La habilidad de la capa límite turbulenta para resistir la separación es tanto mejor a medida que crece el número de Reynolds.

La aerodinámica de bajos Reynolds está estrechamente vinculada con aviones monomotores, planeadores, palas de generadores eólicos y dispositivos clásicos de hipersustentación (flaps Fowler, por ejemplo), entre otros. Los perfiles de bajos Reynolds son aquellos diseñados específicamente para alcanzar buenas características de sustentación y resistencia dentro del rango de Reynolds mencionado más arriba. La pregunta que surge sobre estos temas es cómo controlar el flujo alrededor de perfiles de bajos Reynolds, buscando por ejemplo, una mejora en su performance. La evolución corriente abajo de una capa de corte y las características de mezcla de la capa de mezcla en la que se transforma, puede ser controlada inyectando perturbaciones adecuadas en la capa de corte.

Existen diferentes tipos de manipuladores de capa de corte utilizados para generar perturbaciones: los pasivos y los activos. Diferentes tipos de perturbación provocan diferentes estructuras en la capa de corte produciendo diferentes propiedades de transporte de la capa de mezcla resultante y determinando una estela con diferentes tipos de turbulencia.

Desde ambos extremos de un mini-flap emergen dos capas de corte que se enrollan en un patrón de vórtices alternantes y contra-rotantes estableciendo lo que se denomina una "inestabilidad absoluta de estela" [13]. Es así que el mini-flap Gurney, actuando como elemento pasivo o activo, podría ser una alternativa en el control de las capas de corte que se van desprendiendo y enrollando en una calle de vórtices manipulando las fuerzas actuantes sobre el perfil. 


\section{CAPÍTULO 1. INTRODUCCIÓN}

\subsection{Organización y Estructura de la Tesis}

La metodología de trabajo se resume de la siguiente manera:

- Investigación bibliográfica.

- Determinación de las actividades experimentales.

- Construcción de los modelos.

- Puesta a punto de los mecanismos de control de flujo.

- Ensayo de los perfiles aerodinámicos en diferentes configuraciones.

- Procesamiento de los datos adquiridos.

- Análisis de resultados y conclusiones.

El objetivo planteado en el plan de doctorado era investigar la influencia de los mecanismos de control sobre la estela cercana de perfiles aerodinámicos. Así, se propuso ensayar los modelos a una velocidad de flujo incidente pre-establecida con intensidad de turbulencia mínima. Cabe aclarar, que no se pretendió estudiar el comportamiento de dichos perfiles frente a la intensidad de turbulencia del flujo incidente siendo esto motivo de trabajos futuros.

Se eligió el perfil NACA 4412 por ser un perfil muy estudiado por diferentes autores, utilizado tanto en aeronaves que operan dentro de la capa límite atmosférica turbulenta como así también en la sección media de palas de aerogeneradores. Los modelos se construyeron en poliestireno expandido de alta densidad recubiertos con material plástico para darle un buen acabado superficial.

Se implementaron diferentes mecanismos de control de flujo en forma pasiva (miniflap fijo) y activa como los del tipo móvil y rotantes. Dichos dispositivos se pusieron a punto antes de ser testeados en el túnel de viento. De esta forma se obtuvieron los parámetros principales como frecuencias máximas y amplitudes de desplazamiento o rotación según el caso.

Para cada ensayo se montó el ala en el túnel de viento, tomada de sus extremos mediante balanzas aerodinámicas fijas a la estructura del túnel. Se procedió a calibrar el anemómetro para comenzar los ensayos. Las primeras pruebas se realizaron con el perfil limpio para conocer la configuración característica de la estela de este perfil. Se continuó con los ensayos correspondientes al perfil con mini-flap Gurney fijo y por último se ensayaron los diferentes mecanismos de control de flujo.

Se realizó un barrido con el anemómetro de hilo caliente detrás del borde de fuga en ciertos puntos definidos en el plano de simetría del ala, adquiriendo las muestras a una frecuencia mayor a la de los fenómenos estudiados. Estos valores se evaluaron y procesaron para determinar qué tipo de estructuras fluido-dinámicas caracterizan cada dispositivo. Se ensayó solamente un ángulo de ataque $\left(0^{\circ}\right)$ siendo este superior al ángulo de sustentación nula. 


\section{CAPÍTULO 1. INTRODUCCIÓN}

Una vez que se obtuvo el conjunto de datos se procedió al procesamiento y análisis de los mismos, para obtener conclusiones o realizar nuevas experiencias mejorando el rendimiento buscado.

\subsection{Principales Aportes}

Es fundamental estudiar la forma en que las estructuras fluido-dinámicas se desarrollan naturalmente detrás del perfil y la interacción de dichas estructuras con las perturbaciones producidas por dispositivos de control de flujo para poder entender la influencia sobre las capas de corte que se forman en el borde de fuga y producen modificaciones en la ubicación del punto de estagnación posterior arrojando como resultado variaciones de downwash en la zona de la estela cercana.

El movimiento oscilante de los mini-flaps, permite la modulación de las estructuras turbulentas detrás del borde de fuga del perfil. La implementación de dichos mecanismos podría estabilizar el flujo alrededor del mismo y en la propia estela, controlando y reduciendo las fuerzas fluctuantes. De esta forma, un aumento de la sustentación y una reducción del incremento de la resistencia pueden ser alcanzados, traducido en una mayor eficiencia aerodinámica.

El uso de mini-flaps Gurney podría tener aplicación directa en el control de pérdida en aviones comerciales de gran porte. Los dispositivos clásicos de hipersustentación pueden ser simplificados sin disminuir su eficiencia, mediante la reducción del peso y complejidad, aumentando la autonomía y haciendo más seguros los despegues y aterrizajes. Por otro lado, si se emplean los mini-flaps en palas de aerogeneradores en forma activa, contribuirán a un buen control de la doble pérdida (double stall) mejorando la eficiencia aerodinámica de dichas palas en situaciones de grandes ángulos de ataque. Esta última aplicación tendrá influencia directa en el rendimiento de las turbinas eólicas, una de las fuentes actuales de generación de energía con recursos renovables (el viento).

Este trabajo se centró en la descripción cuantitativa y cualitativa de las características del flujo medio y sus fluctuaciones en la estela de perfiles con mini-flaps Gurney fijos y móviles situados en la cercanía del borde de fuga.

De esta forma, el aporte significativo de la tesis es el estudio y determinación del tamaño de las estructuras turbulentas producidas por dispositivos de control de flujo en la estela cercana, en nuestro caso mediante mini-flaps Gurney rotantes y móviles, y su influencia en el comportamiento aerodinámico del perfil. Esto nos permitirá comprender las variaciones de los coeficientes aerodinámicos mediante la medición de cargas en trabajos futuros. 


\section{CAPÍtulo 2}

\section{CONCEPTOS GENERALES}

\subsection{Aerodinámica de Perfiles}

Nos referimos a la aerodinámica, como a aquella rama de la física que estudia tanto las leyes físicas que gobiernan el desplazamiento de un cuerpo en el seno de un fluido, como así también, sus aplicaciones. De la infinidad de cuerpos estudiados nos centraremos particularmente en aquellos cuerpos que dotados de dispositivos generadores de fuerzas, permiten su movimiento y estabilidad en el fluido, como lo son las aeronaves. Pero será de especial interés para nosotros una de las partes más importantes de la aeronave destinada a la generación de sustentación, denominada ala. Dichos dispositivos, tienen una configuración geométrica determinada de acuerdo a su misión. Esta configuración está definida por la sección transversal del ala, denominada perfil, como así también por la distribución del mismo a lo largo de la envergadura conformando la planta alar.

Si se utiliza un perfil con gran eficiencia pero con una distribución poco apropiada podría arrojar como resultado un diseño pobre o viceversa. La forma y la inclinación del perfil respecto a la dirección de la corriente de aire son de gran importancia en la distribución de presiones que da origen a fuerzas aerodinámicas. Si consideramos al conjunto de todas las fuerzas actuantes sobre la planta alar como una única fuerza y la descomponemos según la dirección perpendicular a la corriente libre y su paralela, obtendremos dos fuerzas conocidas como sustentación $(L)$ y resistencia $(D)$. Dichas fuerzas se pueden estimar por medio de las siguientes leyes:

$$
\begin{aligned}
& L=\frac{1}{2} \rho U_{\infty}^{2} S C_{L} \\
& D=\frac{1}{2} \rho U_{\infty}^{2} S C_{D}
\end{aligned}
$$


Siendo, $\rho$ la densidad del medio donde se desplaza el cuerpo, $U_{\infty}$ la velocidad del fluido en la zona del ala, $S$ la superficie de referencia alar y $C_{L}$ y $C_{D}$ los coeficientes de sustentación y resistencia del ala respectivamente.

Si se observan dichas ecuaciones, se puede deducir que existen dos maneras posibles desde el punto de vista de la aeronave de aumentar la sustentación: aumentar el ángulo de ataque y por ende el $C_{L}$ o aumentar la velocidad. Pero existe un límite, ya que cuando el ángulo de ataque supera cierto valor puede dar lugar al desprendimiento de flujo en la superficie del ala con la consecuente pérdida de sustentación. Dicho desprendimiento puede iniciarse tanto en el borde ataque como en el borde fuga dependiendo del tipo de perfil utilizado.

Existen asumpciones erróneas acerca del fenómeno que produce sustentación en un perfil aerodinámico o en una planta alar si consideramos un ala finita. Así, la imagen del ala circundada por un flujo más rápido de menor presión arriba y uno más lento abajo es una sobre-simplificación peligrosa que no considera la real estructura fluido-dinámica generada por un ala, como lo es el sistema de vórtices que la rodea. Uno de los errores más comunes es el siguiente: imaginemos dos volúmenes contiguos de fluido que se separan en el borde de ataque de un perfil tomando diferentes caminos, uno de ellos por el intradós y el otro por el extradós. No hay teoría que justifique que ambos volúmenes de fluido vuelvan a encontrarse en el borde de fuga al mismo tiempo. Dicha suposición que suele encontrarse en la literatura clásica no es correcta. En los flujos reales las partículas que fluyen sobre el extradós se adelantan considerablemente a las que fluyen sobre el intradós. El fluido de arriba va mucho más rápido.

Un cálculo de presiones sobre el ala, demuestra que si fuese válido el encuentro simultáneo en el borde de fuga de partículas contiguas antes del perfil las velocidades del extradós de los perfiles normalmente utilizados no alcanzarían a aportar la necesaria diferencia de presiones. Los perfiles basados en esta presunción deberían ser mucho más gruesos de lo que son para que los caminos del extradós e intradós sean lo suficientemente diferentes para aportar la sustentación necesaria. Si queremos ser más precisos, deberíamos reiterar que las fuerzas aerodinámicas que actúan sobre un ala en movimiento a través de un fluido y en particular la sustentación, son consecuencia de la variación en el tiempo de la cantidad de movimiento del fluido que rodea el ala debida a la particular estructura vorticosa generada por el ala. Hay sustentación porque hay un cambio en la velocidad y dirección de movimiento del aire.

Pensemos entonces en variaciones de cantidad de movimiento y en fuerzas que aparecen por acción y reacción: el sistema vorticoso del ala desvía una buena parte del flujo hacia abajo recibiendo una reacción hacia arriba. La influencia del ala se hace sentir hasta una cierta distancia en torno del avión afectando parte del flujo de aire que lo rodea, desviando una parte del flujo hacia arriba (upwash), alguna parte hacia los costados y una parte de singular intensidad de ese flujo hacia abajo (downwash). Todo este sistema fluido-dinámico depende en gran medida como lo mencionamos anteriormente de la sección transversal del ala y de la planta alar. 
Se define entonces como "perfil" a la figura que se forma al cortar un ala con un plano imaginario normal a la envergadura. Lo que vuela no es el perfil sino el ala. No obstante, de la adecuada elección del perfil y/o perfiles que configurarán la planta alar, resultará la eficiencia aerodinámica de la misma.

Dentro de los parámetros geométricos más importantes que tienen influencia en el diseño de perfiles podemos encontrar: el radio de borde de ataque, la curvatura, la distribución de espesores a lo largo de la cuerda $(c)$ y por último, el borde de fuga.

Como se verá más adelante, este trabajo tiene como objetivo el estudio de la estela en perfiles, y como ya se mencionó, el borde de fuga juega un papel importante en el proceso de formación de la misma. Para cuerpos sólidos que tienen bordes afilados tales como los bordes de fugas en perfiles se puede aplicar la conocida condición de Kutta. Kuethe y Schetzer [14] enunciaron dicha condición como sigue:

"Un cuerpo con un borde de fuga afilado que se mueve en el seno de un fluido creará alrededor de sí mismo una circulación con tanta intensidad que mantiene el punto de estagnación posterior en el borde de fuga".

En aquellos fluidos que se desplazan alrededor de cuerpos con bordes afilados la condición de Kutta se refiere al patrón de flujo en el cual el fluido se aproxima a dicho borde desde ambos lados, se encuentra en el borde y luego fluye dejando el cuerpo. No hay fluidos en la realidad que fluyan alrededor del borde y se mantengan adheridos al cuerpo.

Cuando un cuerpo simétrico con curvas suaves tal como un cilindro con una sección oval se mueve con un ángulo de ataque nulo a través de un fluido, este no genera sustentación. Hay dos puntos de estagnación en el cuerpo: uno en el frente y el otro en la parte posterior. Ahora si este cuerpo se mueve con un ángulo diferente de cero a través del fluido, siguen existiendo dos puntos de estagnación, uno en la superficie de abajo cerca del borde de ataque y el otro en la superficie de arriba cerca de la zona posterior. La circulación alrededor de este cilindro es nula nuevamente y no hay sustentación generada a pesar de que haya ángulo de ataque positivo.

Si en cambio un perfil con un borde de fuga afilado comienza a moverse con un ángulo de ataque positivo, los dos puntos de estagnación están inicialmente localizados en la zona de abajo cerca del borde de ataque y en la parte superior cerca del borde de fuga, como en el caso del cilindro. Pero a medida que el aire pasa por la zona de abajo hacia el borde de fuga el fluido debe girar alrededor de este borde y desplazarse en la parte superior hacia el punto de estagnación. Como consecuencia se produce un vórtice de gran intensidad en la parte superior del perfil cerca del borde de fuga. A medida que el perfil se mueve, este se desplaza con dicho vórtice, llamado vórtice de arranque (starting vortex).

Como la intensidad del vórtice de arranque aumenta progresivamente, también lo hace la circulación asociada al perfil causando el incremento de velocidad en la parte 
superior del mismo. El punto de estagnación en la parte superior se mueve progresivamente hacia el borde de fuga. Luego de que el perfil se ha movido una corta distancia, el punto de estagnación de la parte superior alcanza el borde de fuga y el vórtice de arranque se desprende quedando detrás. Con el tiempo, este se disipa debido a efectos viscosos. A medida que el perfil continúa su carrera, el punto de estagnación sigue en el borde de fuga. Los flujos de la parte superior e inferior se unen en el borde de fuga y dejan el perfil viajando paralelos uno respecto del otro. Es aquí donde se cumple la condición de Kutta. Una de las consecuencias de la condición de Kutta es que el aire en la parte superior viaja mucho más rápido que el aire debajo del perfil y por lo tanto ambos flujos nunca se vuelven a encontrar. Cuando un perfil se está moviendo con un ángulo de ataque positivo, la condición de Kutta se ha establecido y por lo tanto hay una circulación alrededor del perfil. De esta forma el perfil genera sustentación y su magnitud es calculada por el teorema de Kutta-Joukowski.

La condición de Kutta nos da ciertos indicios de por qué los perfiles siempre tienen borde de fuga afilado, a pesar de que no es deseable desde el punto de vista estructural o de manufactura. Una aeronave con un ala con borde de fuga suavemente redondeado generaría poca o nada de sustentación.

Es importante mencionar que en las asunciones previas, el fluido se encuentra en todo momento adherido a ambos lados del perfil. Ello no sería posible en un ala sin la implementación de elementos adicionales, si el ángulo de ataque aumenta de tal forma que el fluido no puede copiar la forma del perfil dando lugar al desprendimiento de flujo en el extradós y una consecuente reducción en la sustentación (stall).

Una de las mejoras más importantes en la aerodinámica de perfiles consistió en la alteración de la ubicación punto de estagnación con la consecuente modificación de la circulación introduciendo variaciones en la geometría del borde de fuga. Con el avance tecnológico surgieron nuevas ideas, y entre ellas la implementación de técnicas de control de flujo tanto pasivo como activo. Es así que aquí se estudió la implementación de uno de los dispositivos utilizados como elemento de control de flujo llamado mini-flap Gurney, cuyo efecto es modificar la ubicación del punto de estagnación posterior y como consecuencia la configuración de la estela.

\subsection{Turbulencia}

El estudio de flujos turbulentos es de gran importancia en muy variadas aplicaciones tecnológicas como lo son las ingenierías aeronáutica y naval, ingeniería mecánica y estructural, fenómenos de flujo interno, transferencia, combustión, etc.

Las particulares características de la estructura turbulenta de un flujo suelen estar asociadas a fuerzas aerodinámicas a través de cambios del patrón de flujo, influenciando la sustentación y resistencia. Las pérdidas de cantidad de movimiento generadas por 
producción de remolinos y disipación viscosa son efectos comunes observados en aplicaciones aeronáuticas, navales y flujos internos, entre otros.

Si se pretende mejorar u optimizar un dado problema de ingeniería que involucre turbulencia, será preciso entender y controlar al menos al particular conjunto de estructuras turbulentas que gobiernan los fenómenos de interés. El flujo de un fluido puede discurrir de manera ordenada y predecible en los valores instantáneos de las variables físicas involucradas: la velocidad, densidad, presión y/o temperatura. Si las condiciones iniciales y de contorno permanecen inalteradas en el tiempo, las condiciones de flujo no turbulento aparecerán claramente vinculadas a estas condiciones iniciales y de contorno siendo también invariables en el tiempo o variando de una manera totalmente predecible en el tiempo (oscilaciones periódicas).

En contraste, los valores instantáneos de las variables de un flujo turbulento de ninguna manera aparecen dependientes de condiciones iniciales y de contorno. Dichas variaciones son de carácter impredecible, aleatorio. Esta imprevisibilidad no es fruto de una insuficiencia en las mediciones. Por ejemplo, por más que se efectúen infinitas mediciones en una ruleta, estas no permitirán prever el próximo número a salir. "La aleatoriedad es un comportamiento físico que caracteriza a ciertos sistemas". Tal es así, que los valores instantáneos de las magnitudes turbulentas son impredecibles. Ciertas visualizaciones han mostrado regiones con continuos cambios en los patrones de flujo exhibiendo un aparente flujo instantáneo confuso y desordenado.

Algunas denominaciones comúnmente utilizadas en turbulencia como "turbulencia totalmente desarrollada" requieren de una aclaración. Una "turbulencia totalmente desarrollada" es la que se puede desarrollar sin restricciones impuestas. Restricciones de este tipo serán contornos, fuerzas externas de carácter gravitacional, de flotabilidad, centrífugas, viscosas, eléctricas, magnéticas, etc. Si tenemos un canal por el que circula flujo turbulento no podremos considerar desarrollados a los remolinos cuyo tamaño sea similar al del canal, porque les son impuestas las fuerzas que conducen al fluido por el canal. Tampoco lo serán los remolinos que se desprenden de una hélice. Este razonamiento permite deducir que ningún flujo turbulento es generalmente totalmente desarrollado en las escalas correspondientes a las estructuras de gran energía.

Para escalas más pequeñas la turbulencia puede estar "totalmente desarrollada" siempre y cuando los efectos viscosos no intervengan de manera protagónica. Cabe aclarar, que para vórtices muy chicos el número de Reynolds $(R e)$ puede llegar a ser tan pequeño que los efectos viscosos disipadores de energía cinética sean predominantes. En los experimentos con bajas velocidades con corrientes a través de grillas de alambres delgados el flujo nunca llega a estar desarrollado porque los vórtices que generan los alambres son consecuencia de fuerzas de rozamiento pero los vórtices corriente abajo ya son tan pequeños que con las bajas velocidades implican $R e$ tan bajos que nuevamente priman fuerzas debidas a efectos viscosos. Podemos decir entonces, que la "turbulencia totalmente desarrollada" puede ocurrir para escalas menores que las grandes escalas energéticas pero mayores que las pequeñas escalas disipativas. 
Un análisis más meticuloso permite reconocer sin embargo la existencia de ciertos "tipos de orden" normalmente ocultos en el flujo global. Por ejemplo en algunos flujos pueden reconocerse remolinos elípticos de eje mayor vertical que persisten durante un cierto tiempo antes de deformarse o dividirse. Si bien los valores instantáneos de la velocidad de la corriente de estos remolinos siguen siendo aleatorios, su condición de elipticidad (en este ejemplo) no lo es. Esa elipticidad indica una preferencia geométrica y dinámica en los procesos de transporte. Es un tipo de organización espacial que indica un tipo de orden previsible en el hecho de que los remolinos (del ejemplo) son elípticos. Los flujos turbulentos suelen exhibir regiones con movimientos preponderantemente unidimensionales, y/o bidimensionales y/o generalmente tridimensionales con un amplio conjunto de muy diferentes escalas espaciales y temporales.

En la década que abarca aproximadamente desde mediados de 1960 hasta mediados de 1980 el conocimiento de la turbulencia fue revolucionado gracias al empleo de sofisticadas metodologías de análisis de datos experimentales mediante nuevas técnicas de muestreo condicionado y sofisticadas técnicas de visualización. Se comprendió que en muchísimos casos la turbulencia que contenía una importante porción de la energía cinética estaba organizada en diferentes y particulares estructuras coherentes. Dicha organización ocurre en remolinos de formas y tamaños muy diversos, como pueden ser jets, porciones delimitadas de fluido de muy baja velocidad (low velocity streaks) o con muy diversas fluctuaciones de velocidad a lo largo de superficies (sin giros), etc.

Para explicar más conceptualmente este innovador enfoque utilicemos un ejemplo de la medicina. Imaginemos un paciente con un profundo desarreglo en su sistema cardiovascular. El enfoque antiguo (cualitativamente análogo al antiguo enfoque global estadístico de la turbulencia) se concentraba y limitaba a analizar sus mareos, fatiga, falta de coordinación, desarreglos de la memoria, alteraciones de carácter, tomarle la temperatura y la presión. El enfoque moderno agrega a los procedimientos tradicionales el minucioso estudio fluido-dinámico de la sangre por arterias, venas y músculo cardíaco. Se considerará especialmente la sección libre de los conductos de sangre, su geometría, las características de eventuales limitaciones del flujo debidas a depósitos calcáreos o taponamientos, la forma y movimiento de las válvulas cardíacas, etc. Una cura debe intentarse restableciendo los patrones de flujo normales a través de las modificaciones geométricas locales capaces de gobernar la fluido-dinámica (aumentar la sección de los conductos sanguíneos con stents implantados en lugares críticos, by-pass remplazando conductos tapados).

Es por eso que hoy en día, el enfoque moderno de la turbulencia se concentra en el meticuloso estudio de las muy diversas estructuras turbulentas presentes en el campo fluido-dinámico, sin dejar de lado el tratamiento tradicional. 


\subsection{Control de Flujo}

Desde el comienzo, el hombre nunca ha estado satisfecho con el mundo que lo rodea, y trató de controlarlo o mejorarlo para tener efectos beneficiosos. Esto se aplica hoy en día a casi todas las disciplinas de la ciencia, y por ende, la fluido-dinámica no es una excepción. Desde los primeros tiempos, los fluidos fueron una materia muy atractiva y al mismo tiempo difícil de entender, lo que forzó a los investigadores a mejorar sus habilidades y conocimientos. Aun así después de entender parte del complicado comportamiento de los fluidos, los científicos nunca estuvieron satisfechos y pusieron todo su esfuerzo en controlarlo. Es aquí donde nace la disciplina "Control de Flujo".

Es difícil de proveer una definición de Control de Flujo, pero una que se ajusta con cercanía a este trabajo es aquella enunciada por Fiedler [15]:

"es un proceso u operación a través de la cual ciertas características de un fluido son manipuladas en cierta forma para lograr mejoras o una performance técnica específica".

Esta definición de control de flujo está vinculada por el hecho de que esta manipulación esta efectuada por "la conveniente amplificación interna de pequeñas perturbaciones". La palabra "control" parece ser demasiado estricta o a veces arrogante en el sentido que el fluido es solo inducido a comportarse en cierta forma. Este control es solo el desencadenamiento de las inestabilidades naturales por medio de actuadores de baja potencia, de tal forma que el fluido se desarrolla naturalmente en la forma deseada.

Una introducción excelente y una perspectiva histórica de control de flujo se pueden encontrar en Gad-el-Hak [16]. Allí se observa la división de dos grandes grupos de estrategias de control como lo son el control pasivo y activo de flujo. La diferencia esencial radica en que en el control pasivo no se requiere de potencia adicional entregada al fluido. Este tipo de control, es menos efectivo y más comprometedor, ya que la forma o geometría del cuerpo debe ser optimizada para solo una condición del flujo y no puede ser modificada. En cambio, el control activo es más flexible y efectivo en sus aplicaciones. Al mismo tiempo no es tan intrusivo como lo son los dispositivos de control pasivo y pueden producir incrementos substanciales en la performance para un amplio rango de condiciones de flujo.

Las Figuras 2.1 y 2.2 muestran la división realizada por dicho autor donde se puede observar los dos grandes grupos y las diferentes clasificaciones de acción activa. Dentro del grupo de control activo se diferencian el control predeterminado y el control reactivo. El control activo reactivo es aquel donde una señal de control es tomada de la medición de una variable que caracteriza al fluido, mientras que en el caso predeterminado, el control es independiente de la condición del fluido. El control de flujo reactivo así mismo se divide en alimentación (feed-forward), donde la variable medida y la variable a controlar difieren (aplicando una ley de control); y re-alimentación (feed-back), donde la variable controlada es medida, re-alimentada, y comparada con un valor de referencia. 


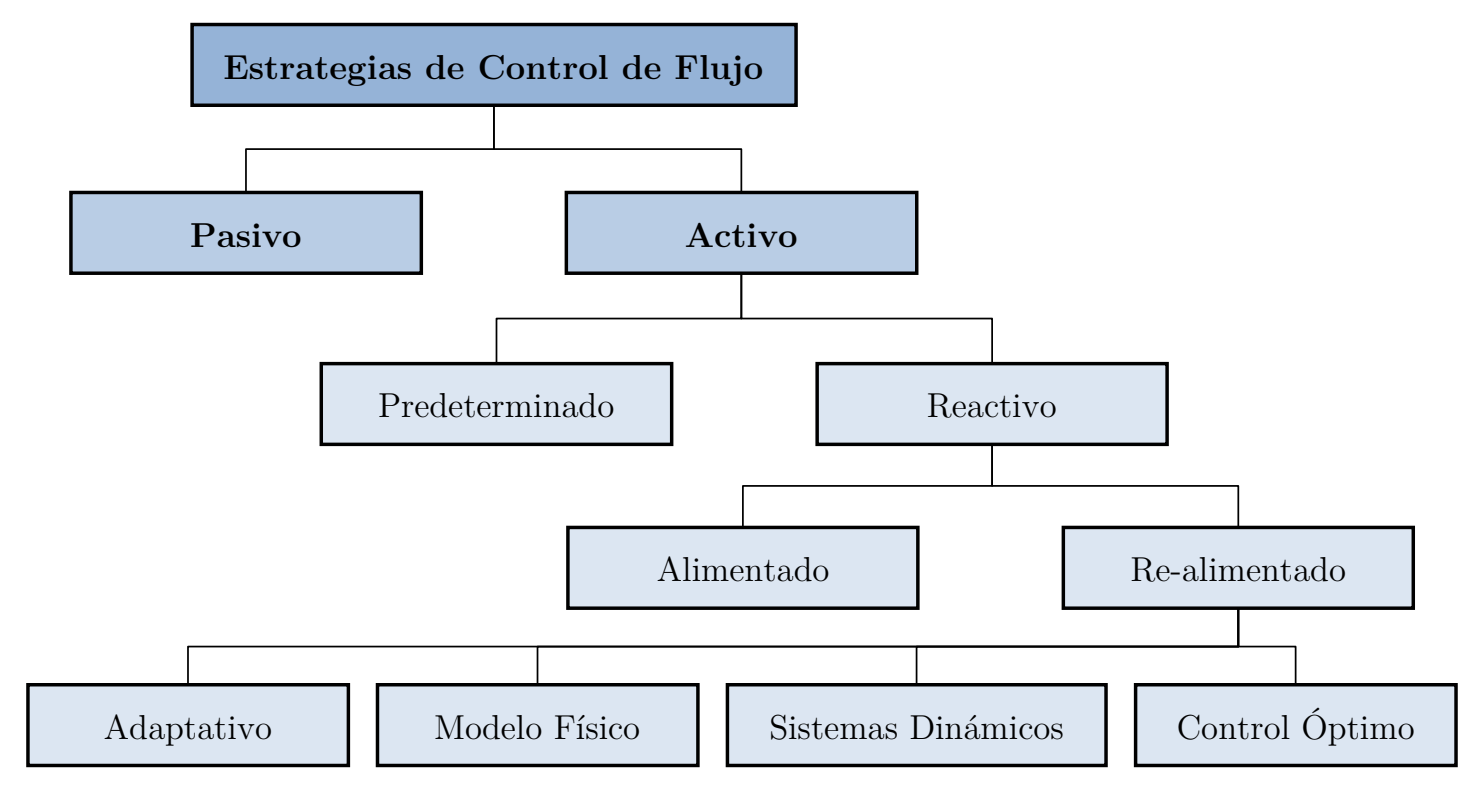

Figura 2.1: Clasificación de las estrategias de control de flujo.

El control activo re-alimentado es también subdividido en diferentes métodos de control de acuerdo al efecto que se desee lograr. Los posibles lazos de control se describen en la Figura 2.2. Allí se muestra una estrategia típica de control predeterminado, siendo esta un simple lazo abierto. El control de flujo activo del tipo reactivo puede ser un lazo abierto de alimentación como en el caso de la Figura 2.3, o un lazo cerrado re-alimentado como en la Figura 2.4.

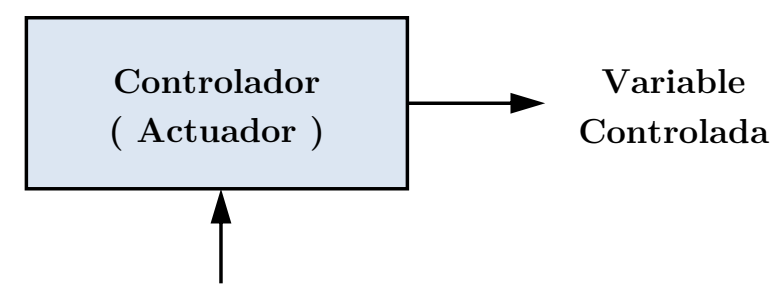

Potencia

Figura 2.2: Control activo de flujo predeterminado. 


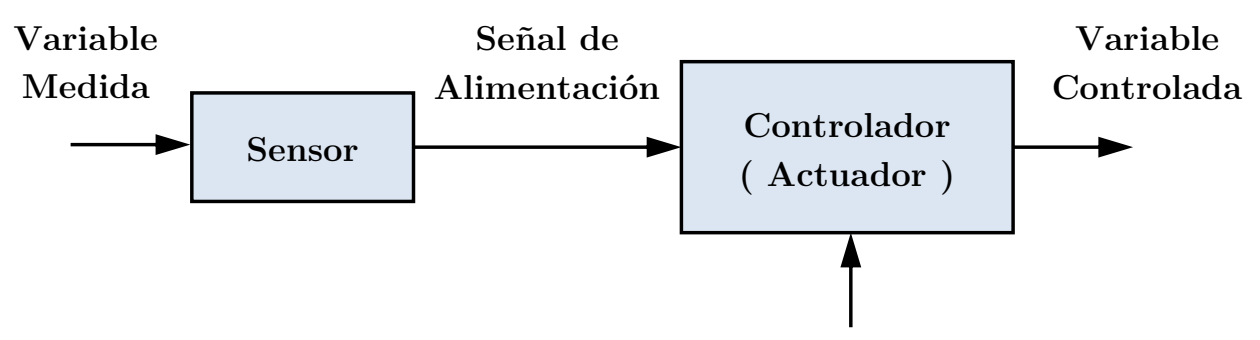

Potencia

Figura 2.3: Control activo reactivo de flujo con alimentación.

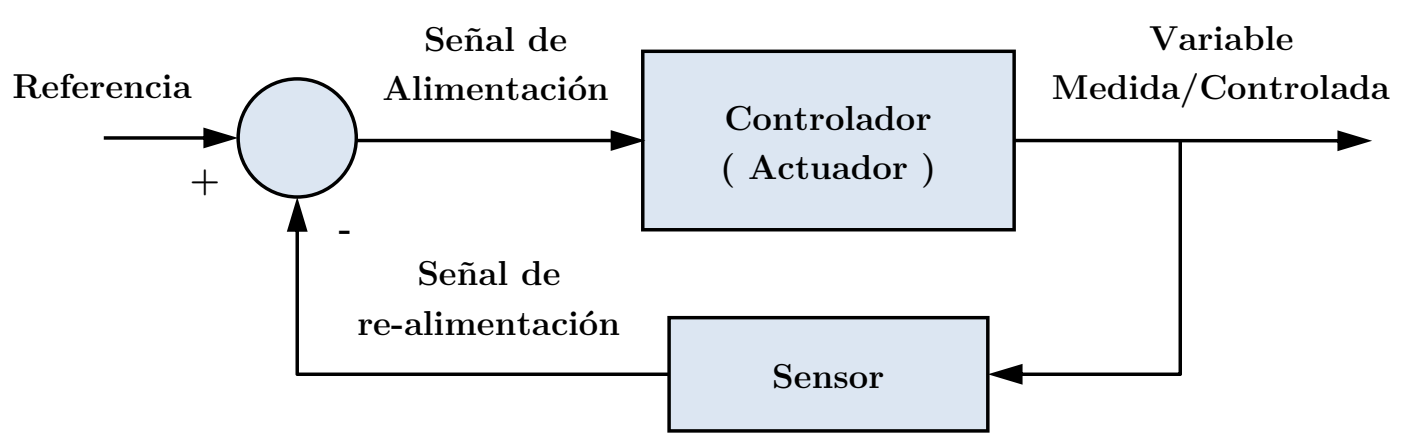

Figura 2.4: Control activo reactivo de flujo con re-alimentación.

Los beneficios de implementar sistemas de control de flujo de forma eficiente sobre un amplio campo de aplicaciones van desde ahorrar miles de millones de dólares en el costo anual de combustible en vehículos terrestres, aéreos y marítimos, hasta lograr procesos industriales donde se ven involucrados los fluidos y que sean tanto económica como ambientalmente más competitivos. Dentro de esta amplia gama de aplicaciones, podemos mencionar la reducción de resistencia y el aumento en la sustentación en aeronaves; el aumento en el intercambio de masa, cantidad de movimiento o energía; la supresión del ruido inducido por el fluido; o una combinación de estos, entre otros. Existen diferentes fenómenos a través de los cuales estos objetivos pueden llevarse a cabo, 


\section{CAPÍTULO 2. CONCEPTOS GENERALES}

como ser adelantar o atrasar la transición de flujo laminar a turbulento, prevenir o provocar el desprendimiento del flujo, o suprimir o aumentar los niveles de turbulencia.

El principal inconveniente de la implementación de control activo de flujo radica en la fabricación e implementación de los actuadores a través de los cuales se logra el control, ya que se busca que dichos dispositivos sean simples, de construcción económica y fáciles de operar, evitando efectos colaterales.

Como se verá más adelante, el tipo de estrategia de control utilizado en este trabajo abarca los dos tipos más simples de estrategias de control, como ser la del tipo pasivo y activo del tipo predeterminado, mediante la implementación de mini-flaps Gurney accionando directamente sobre el actuador sin conocimiento de la condición del fluido. 


\section{CAPÍtulo 3}

\section{METODOLOGÍA EXPERIMENTAL Y EQUIPOS}

\subsection{Introducción}

Durante el desarrollo de la tesis, un conjunto de alas para el túnel de viento con y sin dispositivos de control de flujo fueron construidas. Estos modelos fueron diseñados para poder medir la distribución de velocidades en la estela mediante la técnica de anemometría de hilo caliente. Dicha técnica fue utilizada para estudiar las modificaciones introducidas cuando los mecanismos de control de flujo son desplegados a diferentes frecuencias. La descripción relativa al equipamiento utilizado en los experimentos se provee en las siguientes secciones. Una valiosa fuente de información en relación con la construcción, instrumentación y montaje para ensayos en túnel de viento puede ser encontrada en Barlow et al. [17].

\subsection{Ensayos en Túnel de Viento}

Los ensayos realizados en el túnel de viento se dividieron en varias etapas de acuerdo al programa propuesto para el desarrollo de la tesis. Estas etapas se organizaron como se muestra a continuación:

- Medición de estela en perfil sin dispositivos de control de flujo (perfil limpio).

- Medición de estela en perfil con mini-flap Gurney fijo (control pasivo).

- Medición de estela en perfil con dispositivos de control activo de flujo (mini-flap Gurney móvil y rotante).

\subsection{Construcción de Modelos}

De acuerdo a lo propuesto en el apartado anterior, se construyeron tres modelos para cumplir con la totalidad de los ensayos. En los tres modelos se realizaron las mediciones de perfil "limpio", con mini-flap Gurney "fijo" y mini-flap Gurney "móvil" y "rotante" según el caso. La forma de construcción es similar en cada uno de los modelos pero con 
leves modificaciones de acuerdo al tipo de mecanismo de control de flujo implementado. El proceso de construcción se detalla a continuación.

Un perfil NACA 4412 (Figura 3.1) de 50cm (mini-flap rotante y rotante variante $30^{\circ}$ ) y $60 \mathrm{~cm}$ (mini-flap móvil) de cuerda fue utilizado como perfil base de los modelos. Las coordenadas del perfil se detallan en el Apéndice A. La elección del perfil se basó en la predisposición del mismo a la implementación de dispositivos de control debido a su geometría y a la aplicabilidad en modelos reales. Este perfil es comúnmente utilizado en la sección media de palas de aerogeneradores, y dado que es un campo en constante crecimiento, muchos estudios ya han sido realizados acerca de la implementación de dispositivos de control de flujo. Así mismo, el espesor del perfil cerca del borde de fuga es de dimensiones considerables lo cual favorece el emplazamiento de mecanismos.

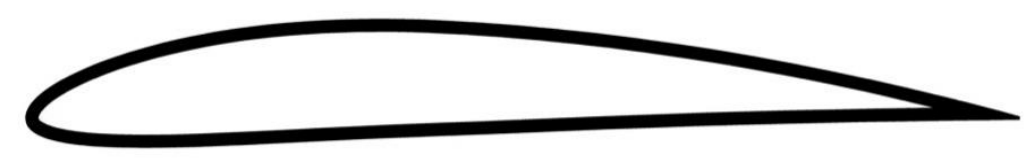

Figura 3.1: Perfil NACA 4412.

Con los datos de la distribución de puntos correspondientes al extradós e intradós, se cortaron con técnica de laser dos matrices de madera del tipo fibro-facil de $3 \mathrm{~mm}$ de espesor. Con esta técnica se pretendía mantener fidelidad en la geometría real del perfil. Las dos matrices se adhirieron a una plancha de poliestireno expandido de densidad $20 \mathrm{Kg} / \mathrm{m}^{3}$ con dimensiones mayores a las de perfil. Luego se procedió al cortado del poliestireno mediante un hilo caliente copiando la forma de los perfiles de fibro-facil. Con el modelo cortado, se comenzó a revestir mediante un material plástico denominado "alto impacto" de 0,5mm de espesor. Este material se pegó a la superficie con adhesivo de contacto logrando un buen acabado superficial. Por último se colocó un tubo de acero inoxidable de un diámetro inferior al espesor del perfil por medio del cual se transmiten las cargas y se fija la posición del ángulo de ataque en caso de que se quieran medir fuerzas aerodinámicas.

Para situar los mecanismos de control de flujo en el interior de los modelos se realizaron cavidades a lo largo de la envergadura para poder ubicar los componentes de despliegue del mini-flap. En la Figura 3.2 se observa el modelo de poliestireno con las placas de fibro-facil a ambos lados luego del corte con hilo caliente. 


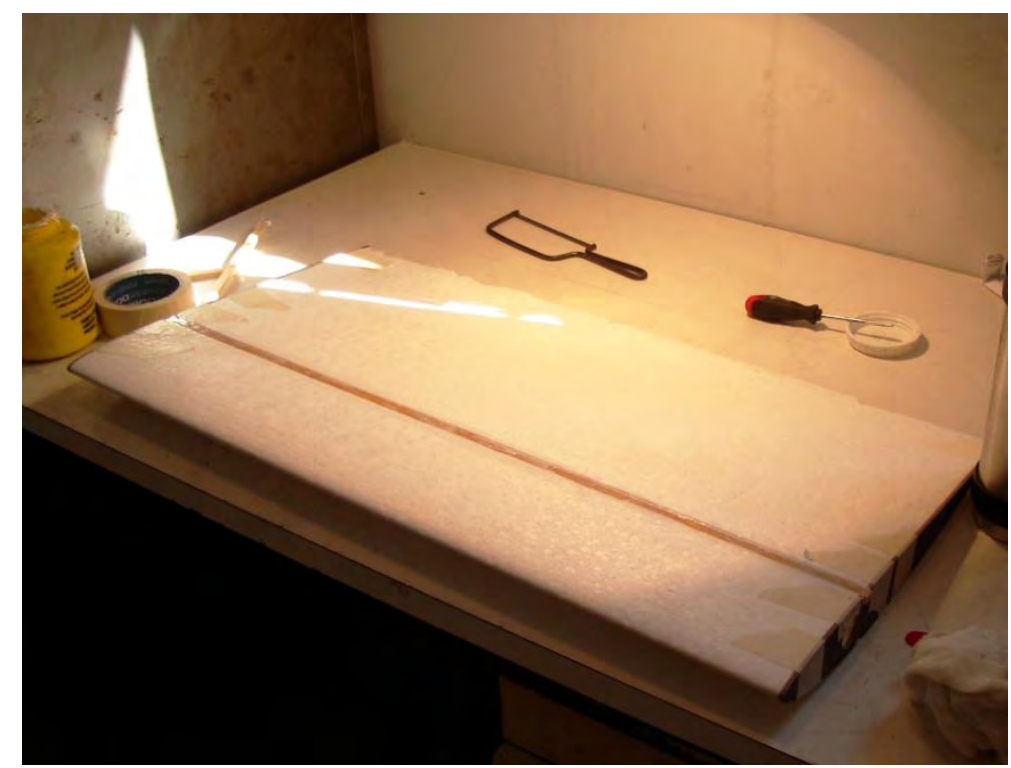

Figura 3.2: Modelo en fase de construcción.

\subsection{Sistemas de Actuación}

Los sistemas de actuación pueden ser divididos en dos grupos: mini-flap Gurney "rotante" y "móvil". Se intentaron diferentes dispositivos con la idea de perturbar la estela del perfil de formas variadas.

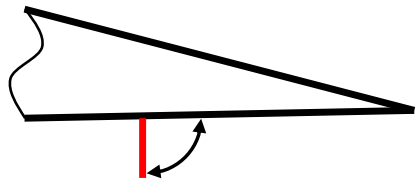

Rotante

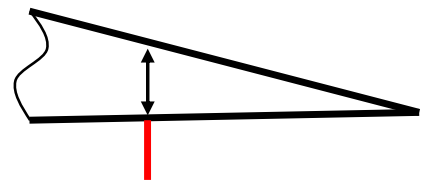

Móvil

Figura 3.3: Movimientos de despliegue de mecanismos.

La diferencia entre ambos mecanismos radica en el modo que el dispositivo es desplegado. En el primero, el mini-flap Gurney se despliega en forma rotativa con un movimiento de vaivén y una amplitud máxima de $90^{\circ}$ para el primer modelo y de $30^{\circ}$ para el segundo modelo. En cambio en el tercer modelo, el mini-flap Gurney se desplaza en movimiento ascendente y descendente, con una amplitud máxima de $8 \mathrm{~mm}$ desde la superficie del intradós. Cuando el mini-flap Gurney asciende, se oculta completamente en el espacio que conforman las superficies superior e inferior del modelo. Es de destacar que todos los mecanismos específicos para los movimientos de los mini-flaps fueron diseñados 
y construidos como parte de trabajo de investigación. La forma en que ambos dispositivos se accionan se observa en la Figura 3.3 y se describen a continuación.

\subsubsection{Mini-flap Gurney Rotante}

El mecanismo del mini-flap rotante consiste en un perfil extrudado de aluminio con una sección tipo "L" colocado a lo largo de la envergadura y capaz de rotar alrededor de un eje ubicado en el vértice del mini-flap y fijo al modelo. En la Figura 3.4 se observa un esquema simplificado del mecanismo. Un motor tipo "Astro Cobalt-05" de corriente continua con una capacidad máxima de rotación sin carga de 16000RPM, tiene adosado al eje un cigüeñal con una articulación para transmitir el movimiento. Una biela unida a la articulación transforma el movimiento de rotación del motor en movimiento traslacional. En la parte superior del mini-flap hay una segunda articulación que toma la biela por el otro extremo y convierte nuevamente la traslación en rotación. La cinemática del mecanismo está diseñada de tal forma que el movimiento final es una rotación alternada con una amplitud máxima de $90^{\circ}$. En este modelo la amplitud de rotación es fija, pero el sistema permite ajustar la frecuencia de despliegue del mini-flap Gurney variando las RPM del motor. La frecuencia máxima alcanzada por el sistema es de $44 \mathrm{~Hz}$.

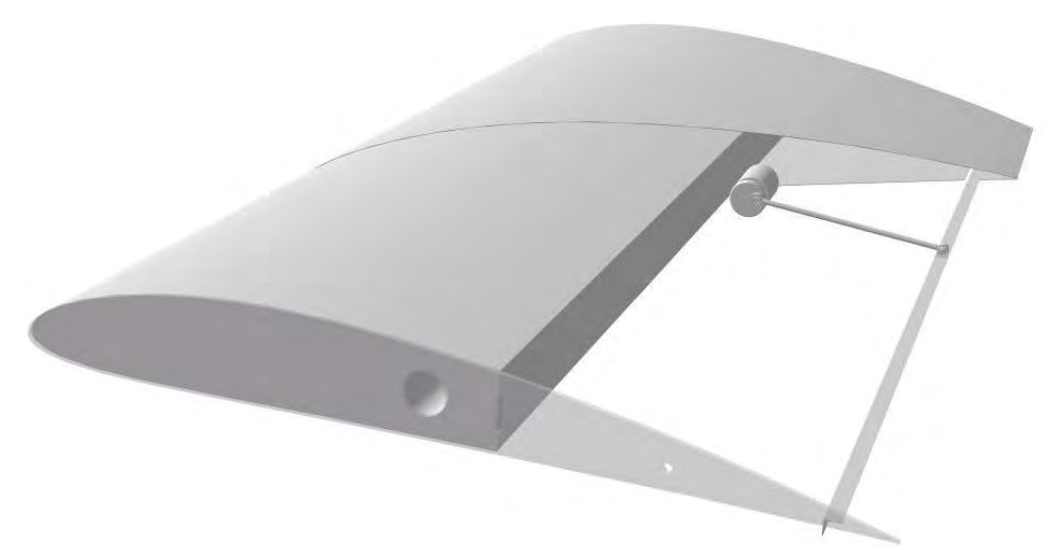

Figura 3.4: Esquema del mecanismo mini-flap Gurney rotante.

\subsubsection{Mini-flap Gurney Rotante (variante $30^{\circ}$ )}

La variante del mecanismo del mini-flap Gurney rotante se construyó con la idea de mejorar las prestaciones del caso anterior. La cinemática de este sistema incorpora más elementos que transmiten el movimiento al mini-flap Gurney pero permiten reducir las vibraciones en el modelo. 
El mecanismo es similar al primer diseño donde un motor de altas prestaciones transmite el movimiento rotacional a lineal y luego transformado a rotacional nuevamente mediante un conjunto de bielas. En el esquema siguiente (Figura 3.5) se presenta un diagrama de la cinemática del mecanismo descrito.

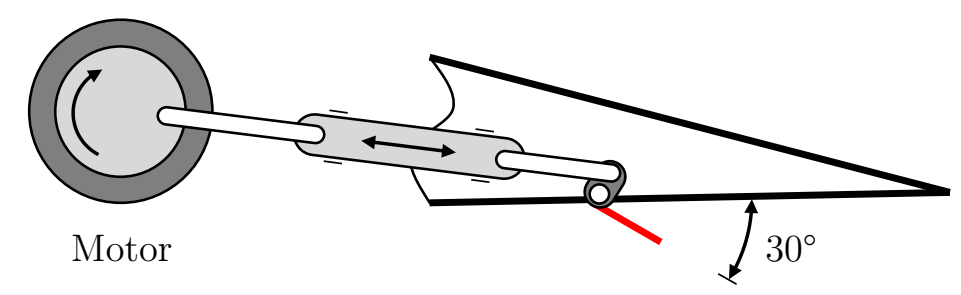

Figura 3.5: Esquema del mecanismo rotante alternativo.

En este caso, el mini-flap de aluminio de $10 \mathrm{~mm}$ de altura se encuentra adosado a un eje de bronce de $3 \mathrm{~mm}$ de diámetro en forma tangente y a lo largo de la envergadura. La amplitud de rotación es de $30^{\circ}$ fija con la posibilidad de modificar la frecuencia cambiando la velocidad de rotación del motor. La frecuencia máxima alcanzada por el sistema es de $50 \mathrm{~Hz}$.

\subsubsection{Mini-flap Gurney Móvil}

Este dispositivo basa su funcionamiento en un sistema electro-mecánico compuesto por un motor de altas prestaciones que acciona un balancín al cual se encuentra adosado el mini-flap Gurney.

Respecto del sistema electromecánico ubicado en su interior, se trata de un listón de balsa, de sección triangular y $780 \mathrm{~mm}$ de longitud, el cual se laminó con fibra de vidrio y resina, incluyendo un tubo de carbono hueco, vinculado a lo largo de la balsa sobre el espesor máximo (el espesor mínimo es el "borde de fuga" de la balsa triangular). Del lado del espesor mínimo se continuó con el laminado en dirección perpendicular, configurando el mini-flap Gurney de altura $1.3 \% \mathrm{c}(\mathrm{c}=600 \mathrm{~mm})$ equivalente a $8 \mathrm{~mm}$.

El tubo de carbono funciona como eje, anclado al ala con cuatro rodamientos blindados de altas RPM, los cuales permiten movimientos rotativos con mínimo roce. Del centro de la basa laminada, en el mismo plano, se extiende una pieza triangular de $3 \mathrm{~cm}$ de lado. De uno de sus vértices continua una varilla de acero delgada de $2.5 \mathrm{~mm}$ de diámetro y $30 \mathrm{~mm}$ de longitud. Debajo de la mencionada pieza hay dos pequeños resortes, paralelos, cuyo objetivo es dotar de "recuperación" al sistema cuando es accionado por el motor. 


\section{CAPÍTULO 3. METODOLOGÍA EXPERIMENTAL Y EQUIPOS}

Sobre la varilla actúa un pequeño cigüeñal vinculado al eje de un motor eléctrico de corriente continua de $200 \mathrm{~W}$, con un voltaje máximo de $15 \mathrm{~V}$. En el perno del cigüeñal hay un pequeño rodamiento blindado de altas RPM que transmite el movimiento rotatorio del motor haciendo oscilar la pieza de balsa laminada con un consecuente movimiento ascendente-descendente del mini-flap Gurney. La velocidad de giro del motor es regulada por un circuito electrónico que genera una señal cuadrada y que luego es amplificada. Dicha señal tiene una amplitud constante de 12Volts y mediante un potenciómetro se ajusta el ciclo de actividad de la señal permitiendo variar las RPM del motor y de esta forma la frecuencia oscilatoria vertical del mini-flap Gurney.

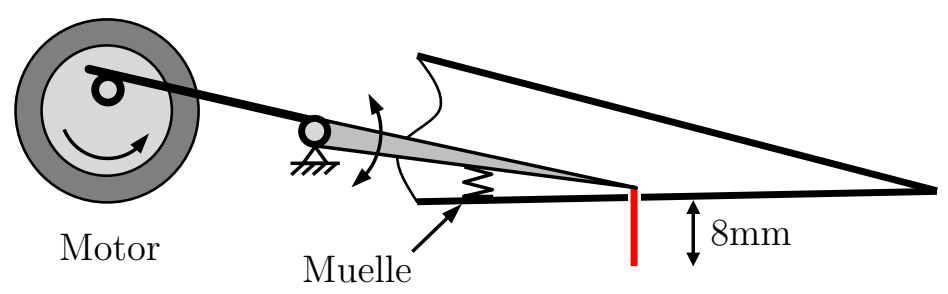

Figura 3.6: Esquema del mecanismo del mini-flap Gurney móvil.

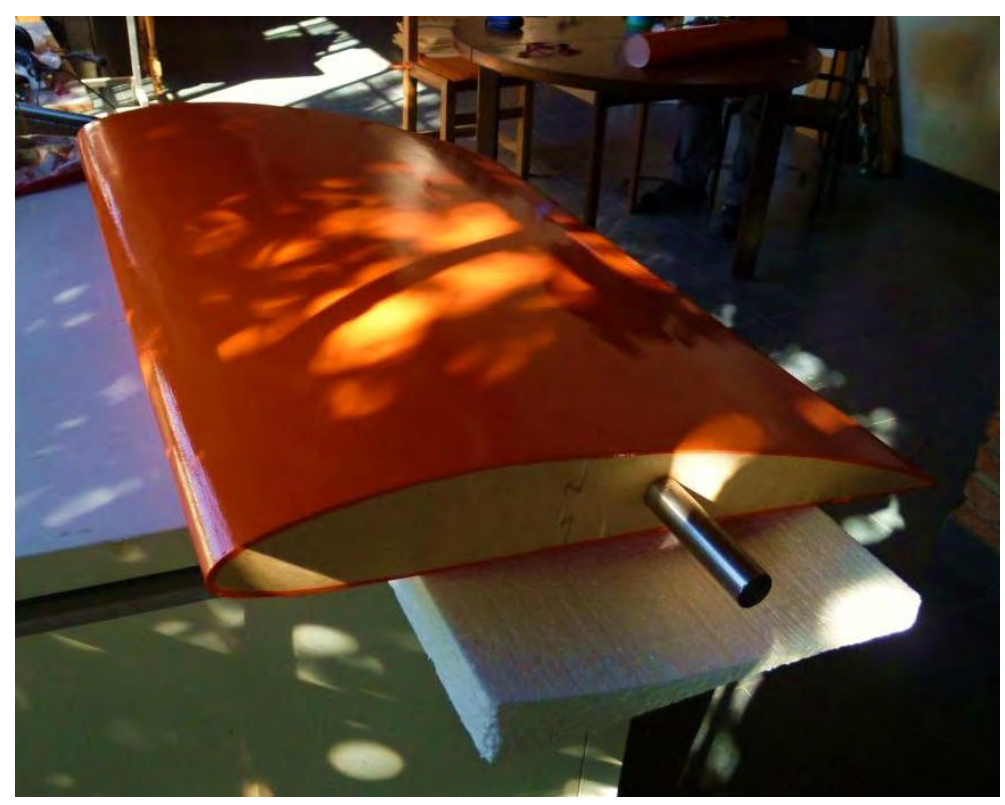

Figura 3.7: Modelo de ala con mini-flap Gurney móvil finalizado. 
El conjunto balancín-mini-flap se diseñó de forma tal de utilizar la menor cantidad de material posible para de esta forma reducir la masa. Como es sabido la masa juega un papel preponderante en el diseño de sistemas mecánicos vibrantes, y nuestro caso no escapa a ello. Es por ello que debido a la naturaleza del sistema la frecuencia máxima alcanzada es superior a los $50 \mathrm{~Hz}$ con una amplitud máxima de $8 \mathrm{~mm}$ y bajos niveles de vibración.

En la figura 3.6 se puede apreciar un esquema del mecanismo interior con el que se encuentra equipado el modelo. Así mismo, en la figura 3.7 se observa el modelo finalizado.

\subsection{Túnel de Viento}

El túnel de viento del Laboratorio de Capa Límite y Fluido-dinámica Ambiental es un túnel de circuito cerrado de bajas velocidades construido en 1968 y cuya sección de prueba actual fue modificada en el año 1984 para poder reproducir la distribución de velocidades en la baja capa límite atmosférica. Para el control de la turbulencia del fluido incidente, una grilla tipo panal de abeja y un set de perfiles paralelos al piso y capaces de rotar a lo largo de un eje fueron incluidos aguas arriba de la sección de prueba. En el piso del túnel, se pueden ubicar elementos prismáticos de espaciado regular, cuyo objetivo es la simulación de la "rugosidad" dada por edificación, arboleda, etc., lo que le imprime características propias a la baja capa límite atmosférica turbulenta.

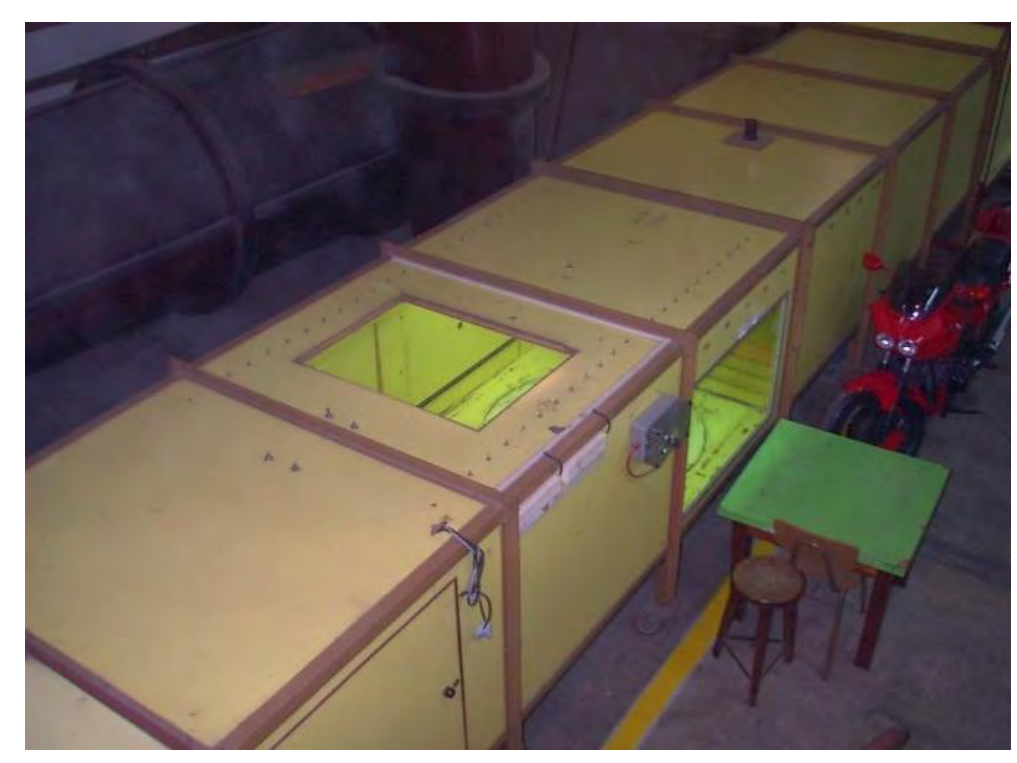

Figura 3.8: Sección de prueba del túnel de viento. 
En el presente trabajo no se deseó estudiar el efecto de flujos turbulentos incidentes sobre el modelo por lo que no se hace uso de los deflectores aguas arriba para cambiar las condiciones del flujo. De este modo los deflectores se ubican en forma paralela al piso del túnel y sin rugosidad en la superficie lo que nos da la condición de uniformidad de velocidades en altura y con la mínima intensidad de turbulencia, llamándose a esta condición "túnel limpio". Un esquema de la sección de prueba se muestra en la Figura 3.8 .

La sección de prueba es de $1 \mathrm{~m}$ de alto por $1.4 \mathrm{~m}$ de ancho y $2 \mathrm{~m}$ de largo, donde una de las paredes laterales incluye una ventana de vidrio para visualización. Otra ventana pero de dimensiones menores se ubica en el techo para colocar fuentes de iluminación y captura de fotografías y videos. Una puerta en la zona posterior permite el acceso a la zona de pruebas. Este túnel de viento no posee ni tobera ni difusor conservando aproximadamente constante el área de la sección transversal a lo largo del circuito. Un anemómetro de hilo caliente modelo "Dantec Flow-Master" puede ser montado a una altura de referencia aguas arriba de la sección de prueba para tomar la lectura de velocidad de la corriente incidente de aire como así también el valor de temperatura. Este equipo tiene un rango útil de medición que va desde 0 a $30 \mathrm{~m} / \mathrm{s}$ con una resolución de $0.1 \mathrm{~m} / \mathrm{s}$ en dicho rango y un error máximo de $5 \%$. La velocidad del fluido en la corriente libre puede variar desde 0 a $20 \mathrm{~m} / \mathrm{s}$ siendo forzado mediante un ventilador conducido por un motor de corriente continua de 50HP. Tanto el motor como el ventilador están montados en una base de concreto para reducir la transmisión de vibraciones a las paredes del túnel.

La velocidad promedio del fluido generada en el túnel es estable en el tiempo con una intensidad de turbulencia mínima de 1,8\%.

\subsection{Equipos de Medición}

\subsubsection{Anemómetro de Hilo Caliente}

La teoría general del funcionamiento de un anemómetro de hilo caliente, está sustentada por las leyes termodinámicas de la transferencia de calor entre el alambre y el fluido, evidenciada por las variaciones de la resistencia del alambre con la temperatura. La pérdida de calor desde el alambre, inmerso en una corriente de fluido, es un proceso físico muy complejo en el que intervienen varios fenómenos al mismo tiempo: conducción, radiación, convección libre y convección forzada. En la mayoría de las aplicaciones en gases, en los que la diferencia de temperaturas entre el sensor y el fluido son menores a $300^{\circ} \mathrm{C}$, la radiación puede despreciarse. De esta manera, la pérdida de calor dependerá de los siguientes factores:

- La velocidad del aire.

- La diferencia de temperaturas entre el aire y el alambre.

- Las propiedades físicas del medio (aire). 
- Las dimensiones y propiedades físicas del alambre.

- La compresibilidad del aire.

- El ángulo entre la dirección del flujo medio y el alambre.

Un anemómetro de hilo caliente modelo "DANTEC Stream-Line" fue utilizado como instrumento de medición de velocidades de flujo en la estela del perfil. El principio de funcionamiento es del tipo CTA (Constant Temperature Anemometer). El equipo permite la colocación de hasta seis módulos independientes e intercambiables lo que facilita su mantenimiento o reposición, más un módulo interno correspondiente al sensor de temperatura. Cada módulo puede ser asociado a un canal o hilo caliente pudiendo conformar combinaciones entre puntas simples, dobles o triples. En la Figura 3.9 se puede observar el equipo con los módulos incorporados.

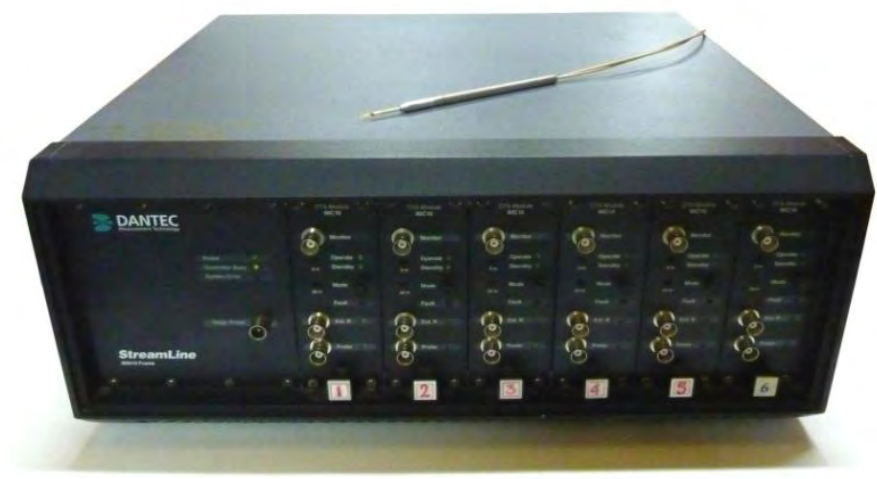

Figura 3.9: Anemómetro DANTEC Stream-Line.

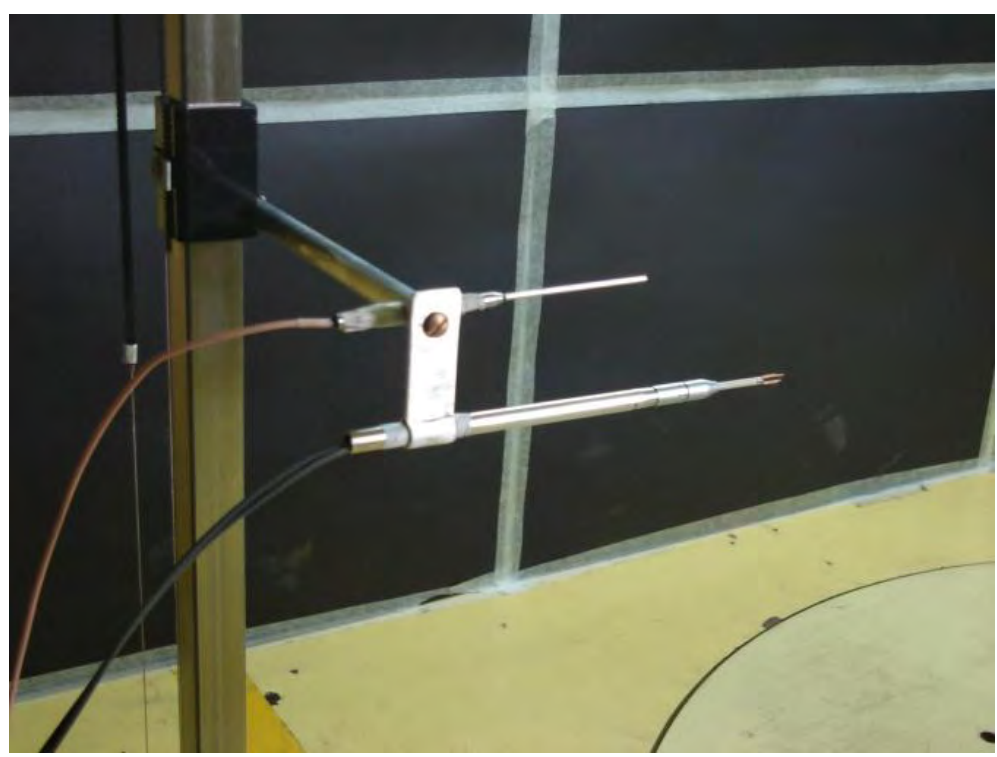

Figura 3.10: Sensores de velocidad y temperatura. 


\section{CAPÍTULO 3. METODOLOGÍA EXPERIMENTAL Y EQUIPOS}

Existe una diversidad de sensores con diferentes especificaciones y aplicaciones compatibles con el equipo antes mencionado. En nuestro caso, para la caracterización del flujo en la estela del perfil utilizamos sensores tipo 55R51 (DANTEC). Este sensor tiene un rango de velocidades que va de 0 a $250 \mathrm{~m} / \mathrm{s}$ y una respuesta en frecuencia de hasta 10 $\mathrm{KHz}$, lo que nos da un amplio rango de trabajo. También se utilizó un sensor de temperatura cuya finalidad es compensar las variaciones de temperatura de los sensores de hilo caliente. La Figura 3.10 muestra ambos sensores montados en el posicionador vertical. En el Apéndice B pueden encontrarse las especificaciones técnicas en forma detallada.

Todo el sistema de medición de velocidades instantáneas cuenta con los siguientes elementos:

- Sensor de velocidad.

- Soporte de sensor.

- Cable sensor-anemómetro.

- Equipo de tratamiento de señal.

- Cable anemómetro-placa adquisidora.

- PC con el software de adquisición.

El sistema de adquisición es una placa de conversión analógico/digital de 16 bits con 16 canales y permite muestrear señales con una frecuencia máxima de $1 \mathrm{MHz}$.

A continuación se puede observar un esquema del conexionado de los diferentes componentes para la adquisición de señales (Figura 3.11) y la disposición de los mismos en el túnel de viento (Figura 3.12).

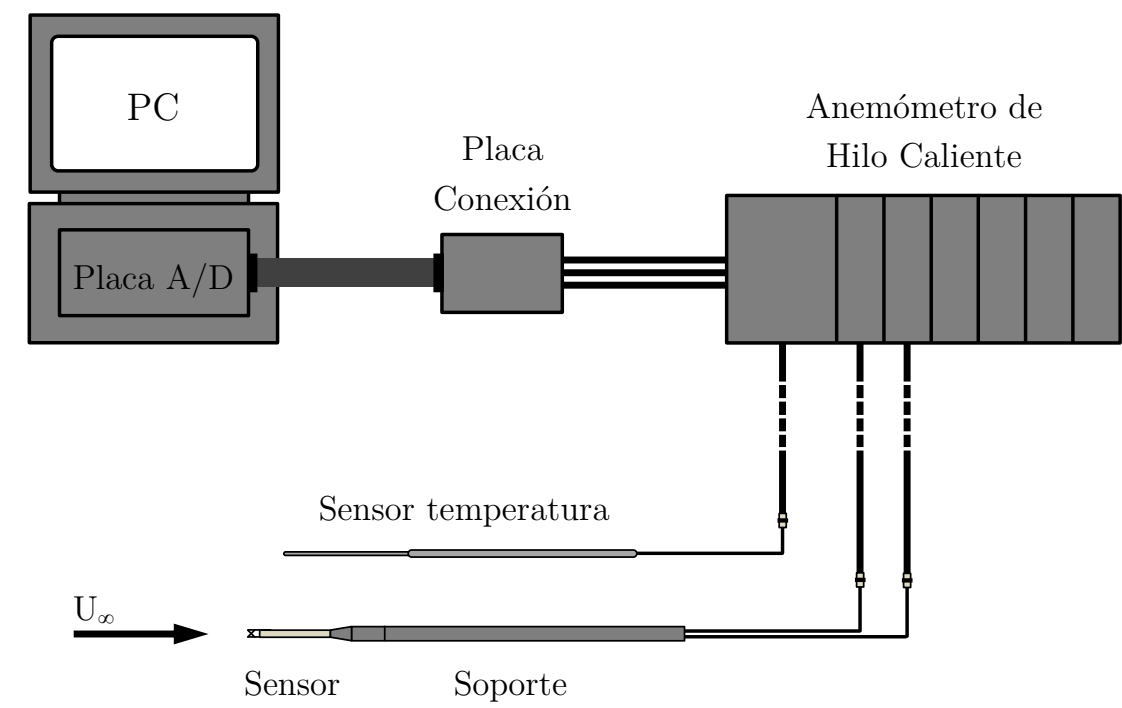

Figura 3.11: Conexionado del sistema de anemometría de hilo caliente. 


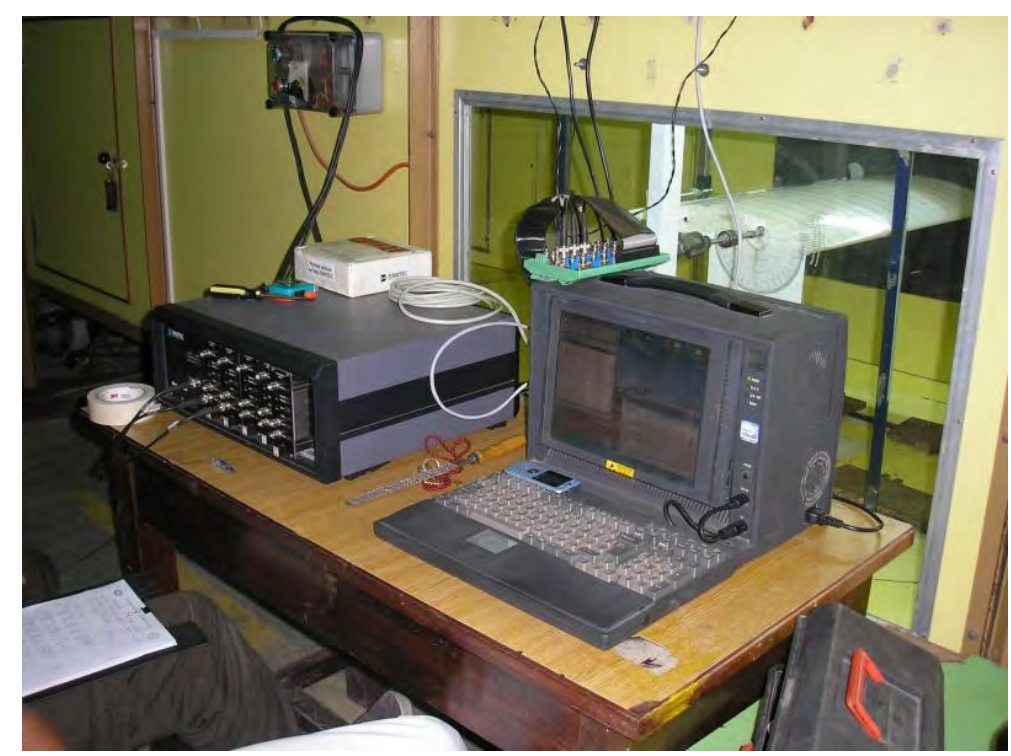

Figura 3.12: Equipamiento en el túnel de viento.

\subsubsection{Posicionador Vertical}

Dicho mecanismo es un dispositivo diseñado ad-hoc con el objetivo de agilizar el procedimiento de medición. Como su nombre lo indica, su tarea es situar la punta anemométrica en la posición de medición adecuada solo en la dirección vertical del túnel de viento.

Está compuesto por un tubo de acero inoxidable de sección cuadrada de longitud similar a la altura de la sección de prueba del túnel. Sobre el tubo, corre una guía de aluminio con un brazo de extensión capaz de sostener tanto el sensor de hilo caliente como el de temperatura. Un motor de pasos con una polea dentada en su eje se ubica en uno de los extremos del tubo. Mediante una correa dentada del mismo paso que la polea, el motor transmite el movimiento a la guía. En el extremo opuesto una roldana le da tensión a la correa para evitar errores en el desplazamiento. Como complemento, dos zapatas se colocaron en los extremos del tubo, de las cuales una de ellas es regulable en altura para permitir la sujeción a las paredes del túnel.

El motor de pasos es alimentado mediante una secuencia coordinada de señales de forma cuadrada para lograr la posición deseada. Dicha señal es generada por el puerto paralelo de un ordenador y luego amplificada para poder brindar la potencia necesaria que demandan las bobinas del motor. Al mismo tiempo, un software con entorno gráfico ad-hoc fue diseñado en lenguaje "Visual Basic". Este programa cumple la función de generar la secuencia de señales para mover el motor y permite ajustar la posición con una 
precisión de $0.02 \mathrm{~mm}$ dada por la relación entre la cantidad de dientes de la polea y la cantidad de pasos por giro del motor.

\subsection{Montaje en Túnel de Viento}

Una vez terminados los modelos, los mismos se colocaron en el túnel de viento para la realización de los ensayos. La disposición debe ser tal que permita la medición de la estela detrás del perfil sin perturbación alguna, ya sea de los elementos en la periferia como así también el mismo sensor de hilo caliente y soporte. Por tal motivo, se tomó la sección central del modelo siendo poco perturbada por la capa límite de los paneles laterales y el posicionador ubicado por detrás del sensor de velocidad y temperatura. Cabe aclarar que la técnica de anemometría de hilo caliente, es una técnica clasificada por varios autores como "intrusiva", y si bien hay errores asociados a la presencia del sensor y soporte, se busca minimizar este efecto con un adecuado montaje.

El ala fue montada en condición empotrada-empotrada y fijada a la estructura del túnel mediante dos balanzas aerodinámicas. Hay que aclarar que la única forma de fijar los modelos es por medio de dichas balanzas y que si bien se han medido cargas aerodinámicas en alguno de los modelos, el análisis de dichos resultados escapa al objetivo de este trabajo siendo motivo de estudios futuros.

El sistema permite la implementación de un mecanismo ad-hoc de cambio de ángulo de ataque con una precisión de $0,025^{\circ}$. El equipo está constituido por un conjunto de engranajes, un motor de pasos y es controlado por un ordenador mediante un amplificador de señal. Conociendo la relación de transmisión y el número de pasos del motor, se puede establecer el mínimo valor de cambio de ángulo de ataque. El engranaje principal esta solidario al tubo central y por lo tanto cuando se aplica una acción en el motor de pasos se verá reflejada mediante dicho engranaje en el ángulo de ataque del perfil. Un software asociado permite ajustar en una interfaz gráfica el valor del ángulo de ataque deseado, pero hay que mencionar que no es un sistema realimentado ya que no existe una medición del ángulo de ataque.

Para asegurar la bi-dimensionalidad del flujo sobre el modelo del ala se colocaron paneles laterales a ambos lados. La idea es evitar efectos de tri-dimensionalidad debido a ángulos de ataque y que la capa límite del túnel de viento perturbe los modelos a ensayar. Supongamos que el modelo se extiende de lado a lado entre las paredes de la sección de prueba. Entonces la capa límite a esa altura sería importante, pero si se colocan paneles de bi-dimensionalidad, la capa límite nace en el borde de ataque de los mismos y por lo tanto el espesor no es de mayor importancia a la altura del perfil. Los paneles tienen una longitud de $1,5 \mathrm{~m}$ y $7 \mathrm{~cm}$ en su máximo espesor. Los primeros $25 \mathrm{~cm}$ corresponden a la geometría de la primera mitad de un perfil simétrico NACA 0012, luego una zona plana de $1 \mathrm{~m}$ de longitud correspondiente a la zona donde se ubica el modelo, y una zona final de $25 \mathrm{~cm}$ que corresponde a la zona de afinamiento. Esta última 
zona es de forma triangular y puede rotar a modo de flap para ajustar la distribución de velocidades en la capa límite del panel. De esta forma, se puede minimizar el error introducido por la interacción de ambas capas límites en la zona de intersección.

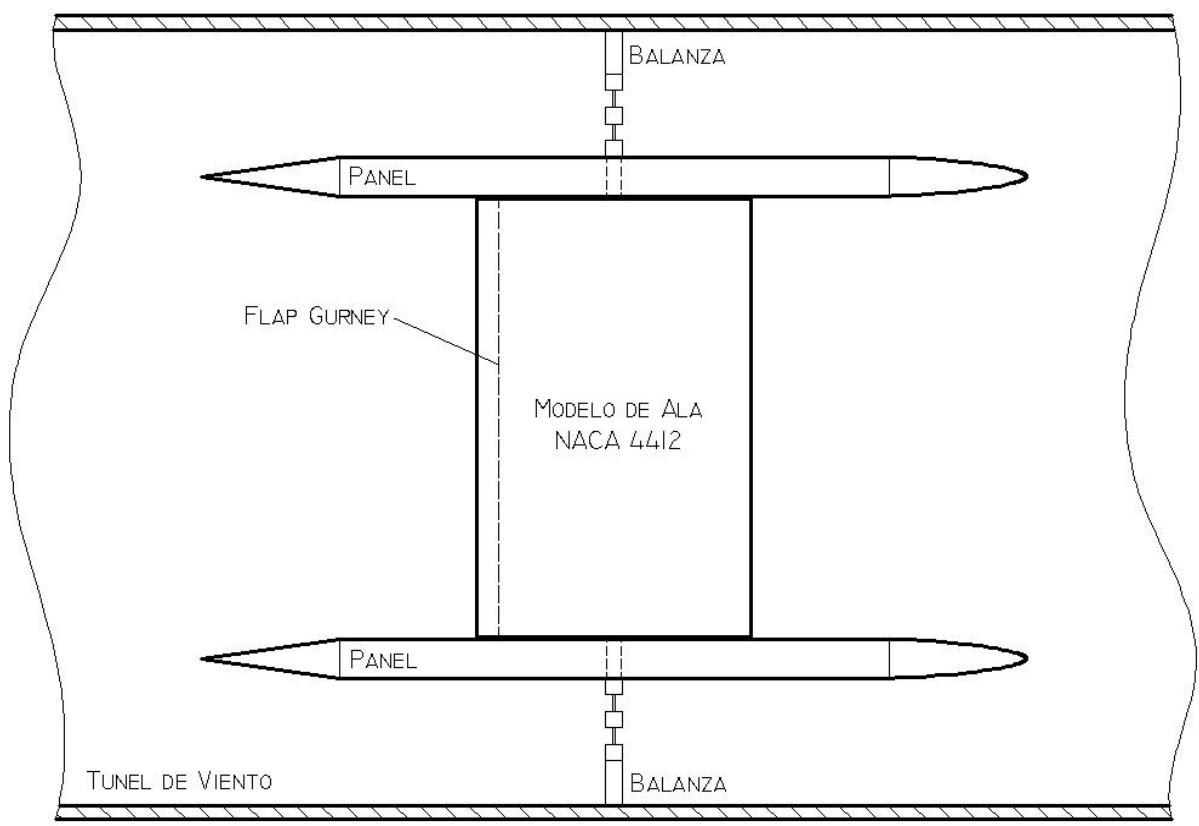

Figura 3.13: Vista en planta del modelo montado en el túnel de viento.

Ambos paneles están construidos con una estructura de hierro soldado, donde uno de ellos está revestido con placas de acrílico para poder visualizar el modelo y el otro con madera pintada de color negro. Ambos lados tienen perforaciones para poder pasar el tubo de fijación. En la Figura 3.13 se muestra un esquema del montaje en el túnel de viento.

\subsection{Metodología de Ensayo}

Una vez finalizada la fase de montaje en túnel, se procedió a la medición de la estela detrás del perfil. Se utilizó la misma metodología de ensayo para todos los modelos testeados. Un resumen del procedimiento se detalla a continuación:

- Fijación de la velocidad de ensayo.

- Ubicación de la punta anemométrica.

- Fijación del ángulo de ataque.

- Ajuste de la frecuencia de mecanismos de control.

- Adquisición y almacenamiento de datos. 
La velocidad de ensayo se puede ajustar por medio del controlador del ventilador y fue medida con el anemómetro de hilo caliente de punta blindada descrito anteriormente. Si bien los ensayos se realizaron a diferentes velocidades, se decidió comparar los resultados para una velocidad solamente.

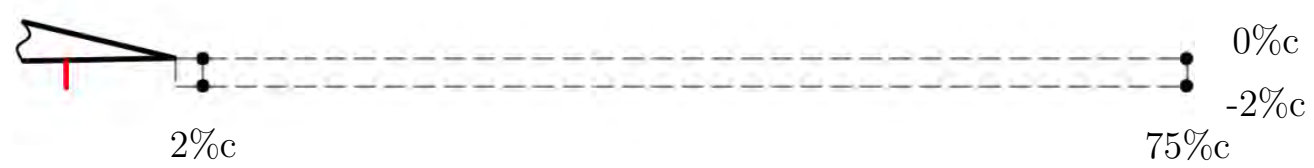

Figura 3.14: Ubicación de los puntos de comparación en el Ala 1.

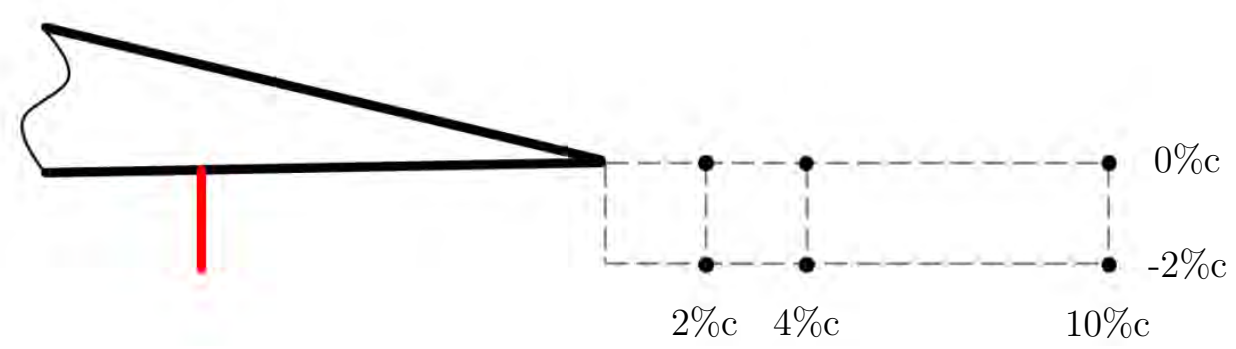

Figura 3.15: Ubicación de los puntos de comparación en el Ala 2 y 3.

La punta anemométrica se colocó de forma tal de captar las estructuras vorticosas más importantes detrás del perfil. De esta forma se decidió ubicarla como ya se ha dicho en la sección media del modelo, a diferentes posiciones aguas abajo y a diferentes alturas por encima y por debajo del borde de fuga. La distribución de posiciones en altura es mucho más densa que la distribución aguas abajo. Es por ello, que se diseñó el mecanismo de posicionado vertical automático dándole eficiencia al proceso de medición. Un esquema de los puntos de comparación se muestra en la Figura 3.14 y Figura 3.15.

De la misma forma, se varía la frecuencia de accionamiento de los mecanismos de control de flujo observando la tendencia de los mismos y que efecto tiene sobre la estela como ser si hay modificaciones en el espectro de potencia, variaciones de las escalas turbulentas e intensidad de turbulencia, entre otras. 


\section{CAPÍTULO 3. METODOLOGÍA EXPERIMENTAL Y EQUIPOS}

Por último, se realizó la adquisición de datos en un lapso de tiempo razonable de modo de capturar las escalas turbulentas más grandes para luego ser procesados por programas de tratamiento de señales. La información se almacenó en el disco rígido de un ordenador. Algunos de los parámetros de adquisición se detallan a continuación:

Mini-flap Gurney Rotante:

- Frecuencia de adquisición $600 \mathrm{~Hz}$.

- Frecuencia de corte de filtro $300 \mathrm{~Hz}$.

- Número de muestras 8192.

- Tiempo de adquisición 13.65seg.

Mini-flap Gurney Rotante (Variante $30^{\circ}$ ) y Mini-flap Gurney Móvil:

- Frecuencia de adquisición $4000 \mathrm{~Hz}$.

- Frecuencia de corte de filtro $2000 \mathrm{~Hz}$.

- Número de muestras 32768.

- Tiempo de adquisición 8.192seg. 


\section{Capítulo 4}

\section{Procesamiento de Datos}

\subsection{Análisis de las Señales}

Los datos analizados en el trabajo corresponden a componentes de la velocidad del viento detrás del mini-flap Gurney, los que son digitalizados y almacenados como secuencias de valores discretos, muestreados a una frecuencia de adquisición de $600 \mathrm{~Hz}$ y $4000 \mathrm{~Hz}$ según el caso.

Un flujo turbulento no es determinístico, es decir, no es posible predecir qué valores instantáneos alcanzará la velocidad medida en un punto dado, por lo que es necesaria una descripción estadística de la variación de velocidad y las demás variables de interés.

Se asume en el análisis que las señales analizadas son ergódicas (distintos segmentos temporales de la señal pueden considerarse equivalentes a distintas realizaciones independientes del experimento aleatorio). Esto implica que para un proceso aleatorio estacionario, propiedades como valor medio, momentos de orden superior y autocorrelación, obtenidas por promediación temporal de valores instantáneos de la señal en intervalos suficientemente largos, no dependen del instante inicial del intervalo [18].

Se definirán y describirán brevemente a continuación las variables y funciones utilizadas en el trabajo para el análisis de los datos de velocidad de viento. En general se definirán para una o dos variables genéricas, $x(t)$ e $y(t)$, separando sus partes media y fluctuante como:

$$
\begin{aligned}
& x(t)=\bar{x}+x^{\prime}(t) \\
& y(t)=\bar{y}+y^{\prime}(t)
\end{aligned}
$$


La barra superior indica que se trata de un valor medio, cuya definición matemática es:

$$
\bar{x}=\lim _{T \rightarrow \infty} \frac{1}{T} \int_{t}^{t+T} x(t) d t
$$

Las estadísticas de una señal pueden agruparse según Bruun [19] de acuerdo al tipo de información que proveen en:

- Estadísticas en el dominio de amplitud.

Son cantidades que brindan información relacionada con la distribución de amplitudes de la señal, pero no dan información sobre su evolución temporal. Dependiendo si tratamos con una única señal o con dos señales podemos encontrarnos con las siguientes funciones:

a) Para una señal $x(t)$ : su función densidad de probabilidad, $p(x)$, el valor medio $\bar{x}$, el valor medio cuadrático de la fluctuación, $\overline{x^{\prime 2}}$ y su raíz cuadrada, el desvío standard $\sigma_{x}$, momentos de orden superior, $\overline{x^{\prime m}}$ y otros parámetros como "skewness" (sesgo) y "kurtosis".

b) Para dos señales $x(t)$ e $y(t)$ adquiridas simultáneamente: además de las estadísticas de amplitud de cada señal, la función distribución de probabilidad conjunta, $p(x, y)$, el valor medio del producto de fluctuaciones, $\overline{x^{\prime} y^{\prime}}$ (que aparece en las tensiones de Reynolds si $x(t)$ e $y(t)$ representan componentes de la velocidad instantánea), correlaciones espaciales, si $x(t)$ e $y(t)$ se obtienen de sensores separados, y momentos cruzados de orden superior, $\overline{x^{\prime m} y^{\prime k}}$.

- Estadísticas en dominio del tiempo.

Son principalmente la función autocorrelación $R_{x}(\tau)$, la correlación cruzada $R_{x y}(\tau)$, y los respectivos coeficientes adimensionales de autocorrelación y correlación cruzada de dos variables. La autocorrelación brinda información sobre el intervalo temporal en el que la señal está correlacionada, y sobre la tasa de decaimiento de esta correlación. 
- Análisis en dominio de frecuencia.

Una forma alternativa, pero complementaria de describir la naturaleza de la señal, es a través de su espectro. Físicamente, esto da una medida de cuánta energía de la señal está contenida en cada banda de frecuencias.

\subsection{Análisis Estadístico de Amplitud de Señal}

\subsubsection{Valor Medio}

Considérese un registro temporal de una función $x(t)$, que puede descomponerse en un valor medio y una fluctuación $x(t)=\bar{x}+x^{\prime}(t)$. Para un registro de duración infinita, la definición de valor medio es como se muestra en la ecuación 4.3.

Cuando se analizan datos digitalizados, como en nuestro caso, la señal continua $x(t)$, $0 \leq t \leq T$, es reemplazada por valores discretos $x_{i}, \quad i=1,2, \ldots, N$, provenientes de un muestreo de la señal, como indica la Figura 4.1. El punto inicial, $t_{o}$, no interviene en las ecuaciones que desarrollaremos. El tiempo total de muestreo es $T$, el intervalo de muestreo es $\Delta t$, y el número total de valores es $N$, de modo tal que:

$$
T=N \cdot \Delta t
$$

La frecuencia de muestreo es la recíproca del intervalo $\Delta t$.

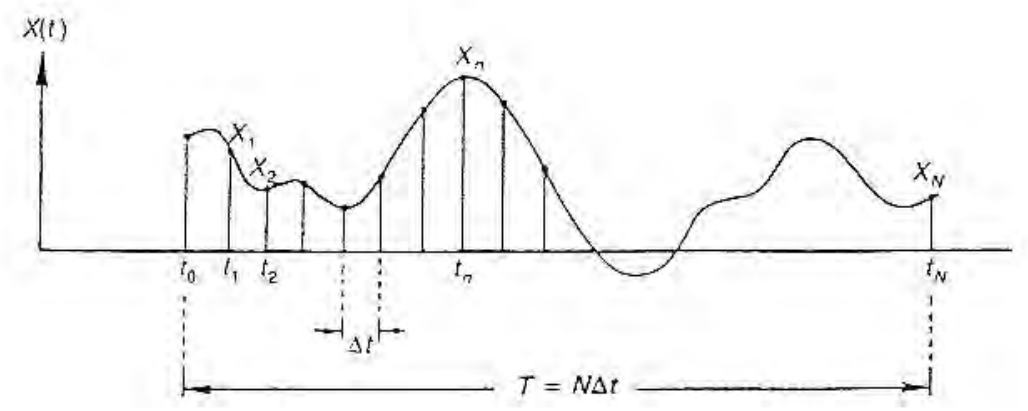

Figura 4.1: Muestreo discreto de una señal continua.

La estimación del valor medio para datos digitalizados es simplemente el promedio de los mismos y se estima de la siguiente forma: 


$$
\bar{x}=\frac{1}{N} \sum_{i=1}^{N} x_{i}
$$

El valor así obtenido es un estimador estadístico no sesgado (el valor esperado del estimador para $N \rightarrow \infty$ coincide con el valor verdadero que se estima) del verdadero valor medio de la función continua $x(t)$. Para acotar el posible error del valor calculado con respecto al valor verdadero, se calcula el desvío standard de $\bar{x}$.

Para un proceso ergódico y $N_{m}$ muestras digitales independientes, el desvío standard de la media de $x(t)$ y el de la función $x(t)$, se relacionan por:

$$
\sigma^{2}(\bar{x})=\frac{\sigma_{x}^{2}}{N_{m}}
$$

Dos segmentos de la señal analizada pueden considerarse independientes si están separados por al menos dos veces el valor de la escala integral de tiempo, $T_{I}$, que se definirá más adelante. En un intervalo de medición de duración $T$, el desvío standard de la media es:

$$
\sigma^{2}(\bar{x})=\frac{2 T_{I} \sigma_{x}^{2}}{T}
$$

\subsubsection{Varianza y Desvío Standard}

La varianza de una señal $x(t)$ se define como:

$$
\sigma_{x}^{2}=\lim _{T \rightarrow \infty} \frac{1}{T} \int_{0}^{T}(x-\bar{x})^{2} d t=\lim _{T \rightarrow \infty} \frac{1}{T} \int_{0}^{T} x^{\prime}(t)^{2} d t
$$

La raíz cuadrada positiva de la varianza, $\sigma_{x}$, es llamada desvío standard. Para una señal digital, un estimador no sesgado de la varianza es: 


$$
\sigma_{x}^{2} \cong \frac{1}{N-1} \sum_{i=1}^{N}\left(x_{i}-\bar{x}\right)^{2}=\frac{1}{N-1} \sum_{i=1}^{N} x_{i}^{\prime 2}
$$

El desvío standard da una medida de la dispersión de la señal en torno a su valor medio. Por ejemplo, si la señal tiene una distribución de probabilidad tipo Gaussiana, el $95 \%$ de los valores instantáneos están en el intervalo $\bar{x} \pm 2 \sigma_{x}$.

De la misma forma y conociendo el valor del desvío standard $\sigma_{x}$ se puede estimar la intensidad de turbulencia $I t_{x}$ para una señal $x(t)$ de la siguiente forma:

$$
I t_{x}=\frac{\sigma_{x}}{\bar{x}}
$$

\subsubsection{Estadística Conjunta de Dos Señales}

El valor medio del producto de las fluctuaciones $x^{\prime}(t)$ e $y^{\prime}(t)$ se define:

$$
\overline{x^{\prime} y^{\prime}}=\lim _{T \rightarrow \infty} \frac{1}{T} \int_{0}^{T} x^{\prime}(t) y^{\prime}(t) d t
$$

La ecuación correspondiente para su estimador en señales discretas es:

$$
\overline{x^{\prime} y^{\prime}} \cong \frac{1}{N} \sum_{i=1}^{N} x_{i}^{\prime} y_{i}^{\prime}
$$

Estos valores son importantes en análisis de flujos turbulentos, ya que típicamente representan tensiones de Reynolds $\left(-\rho \overline{u^{\prime} w^{\prime}}\right)$ o correlaciones temperatura-velocidad que representan transporte convectivo de energía. 


\subsection{Análisis en Dominio del Tiempo}

\subsubsection{Autocorrelación}

La función autocorrelación describe la similitud o dependencia entre los datos tomados en un instante cualquiera $t$ con los datos tomados un tiempo $\tau$ más tarde, en $t+\tau$, para una función $x(t)$ con media 0 . La autocorrelación da el promedio del producto de $x(t)$ por $x(t+\tau)$ para todos los instantes $t$. En una función de media nula (es decir, que toma valores tanto positivos como negativos), es esperable que para pequeños valores de $\tau, x(t)$ y $x(t+\tau)$ sean similares y su producto positivo, mientras que para valores de $\tau$ crecientes, los valores de $x(t)$ y $x(t+\tau)$ tiendan a ser independientes y el promedio de su producto tienda a 0. En el análisis de turbulencia, lo que importa es correlacionar las fluctuaciones de la velocidad, por lo que el valor medio se resta, y se trabaja con registros con media 0 . La definición matemática de la autocorrelación es:

$$
R_{x}(\tau)=\lim _{T \rightarrow \infty} \frac{1}{T} \int_{0}^{T} x^{\prime}(t) x^{\prime}(t+\tau) d t
$$

Por su definición, $R_{x}(\tau)$ es una función real (no necesariamente siempre positiva) y par, es decir $R_{x}(\tau)=R_{x}(-\tau)$. Alcanza su máximo valor en $\tau=0$, donde

$$
R_{x}(0)=\sigma_{x}^{2}
$$

El coeficiente de autocorrelación, $C_{x}(\tau)$ se define adimensionalizando la correlación con su valor máximo:

$$
C_{x}(\tau)=\frac{R_{x}(\tau)}{\sigma_{x}^{2}}
$$

La Figura 4.2 muestra un gráfico típico de $C_{x}(\tau)$. Su valor en $\tau=0$ es siempre 1. La integral del coeficiente de autocorrelación define un valor $T_{I}$, llamado escala integral de la turbulencia:

$$
T_{I}=\int_{0}^{\infty} C_{x}(\tau) d \tau
$$




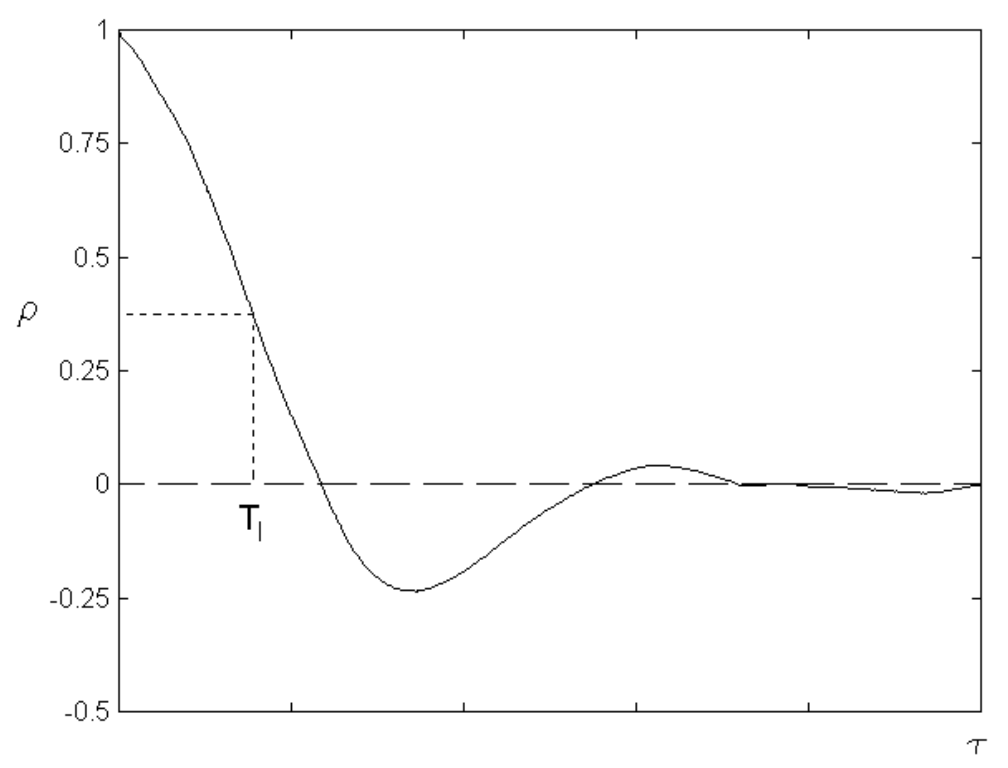

Figura 4.2: Función autocorrelación de una medición de velocidad instantánea de viento.

Esta da una medida del lapso durante el cual los valores $x(t)$ y $x(t+\tau)$ están correlacionados.

En la práctica, una medida de la escala integral de la turbulencia está dada por el tiempo en que el coeficiente de autocorrelación alcanza el valor de $1 / \mathrm{e} \approx 0.37$. Este es exactamente el valor de la integral si $C_{x}$ tiene un decaimiento exponencial, y es una buena aproximación en la mayoría de los análisis de turbulencia [20].

Existen también otras formas de estimar dicha escala tomando el valor de tiempo donde la función de autocorrelación cruza por cero o estimando una pendiente tangente al comienzo de la función y tomando nuevamente el valor de tiempo donde dicha pendiente cruza por cero. El primer y último método (1/e y pendiente) nos dan valores más pequeños que el segundo método (cruce por cero) por lo que podemos decir que estos intervalos de tiempo más pequeños nos dan una idea de las escalas más pequeñas de la señal analizada.

Para una señal digitalizada, adquirida a intervalos $\Delta t$ iguales, donde los valores de $x$ pueden expresarse como $x(n \Delta t), n=0,1,2, \ldots N-1$, un estimador de la función autocorrelación para un retardo $\tau=p$. $\Delta t$ se obtiene de [21]: 


$$
R_{x}(p \Delta t) \cong \frac{1}{N-p} \sum_{n=0}^{N-p} x^{\prime}(n \Delta t) x^{\prime}(n \Delta t+p \Delta t), \quad p=0,1,2, \ldots, m
$$

donde $m \Delta t$ es el máximo retardo considerado, $m<N / 2$.

La coherencia entre la escala temporal de una fluctuación y su escala espacial está implícita en la hipótesis de Taylor de "flujo congelado", o "frozen flow hypothesis". Esta establece que la turbulencia puede considerarse "fija" en un flujo medio que la transporta por convección, como una fotografía que se desplazara con la velocidad media del flujo, y es una aproximación válida en los casos en que el tiempo de evolución de los remolinos o estructuras que la componen es mucho mayor que el tiempo que tarda cada remolino en atravesar el sensor correspondiente. En este caso, si $L_{I}$ y $T_{I}$ son respectivamente las escalas espacial y temporal del flujo, y $U_{\infty}$ es la velocidad media incidente, se cumple:

$$
L_{I} \approx U_{\infty} T_{I}
$$

\subsection{Análisis en Dominio de Frecuencia}

\subsubsection{Análisis de Fourier}

La transformada de Fourier es la herramienta que permite pasar del dominio del tiempo al de la frecuencia, y la anti-transformada permite el paso inverso:

$$
\begin{gathered}
\hat{f}(\omega)=\int_{-\infty}^{\infty} f(t) e^{-i \omega t} d t \\
f(t)=\frac{1}{2 \pi} \int_{-\infty}^{\infty} \hat{f}(\omega) e^{i \omega t} d \omega
\end{gathered}
$$




\section{CAPÍTULO 4. PROCESAMIENTO DE DATOS}

Su aproximación para datos discretos es:

$$
\begin{gathered}
\hat{x}(n)=\sum_{k=0}^{N-1} x_{k} e^{-i 2 \pi n k \Delta t} \\
x(t)=\frac{1}{2 \pi} \sum_{k=0}^{N-1} \hat{x}_{k} e^{i 2 \pi n k \Delta t}
\end{gathered}
$$

En el estudio del viento, estamos interesados principalmente en las frecuencias de las fluctuaciones asociadas a remolinos y otras estructuras vorticosas que conforman la turbulencia.

En el caso del análisis de turbulencia, la función densidad de potencia espectral $S(n)$ o PSD, definida para un intervalo discreto de medición $T$ como:

$$
S_{T}(n)=\frac{|\hat{x}(n)|^{2}}{T}
$$

representa la contribución de una frecuencia $n$ a la varianza total del espectro. Por lo tanto, el espectro nos da una idea de cuánto de la varianza de una señal temporal está asociada a una determinada frecuencia, o a su recíproca, una determinada escala temporal. La varianza total es [22]:

$$
\overline{x^{12}}=\sigma_{x}^{2}=2 \int_{0}^{\infty} S(n) d n
$$




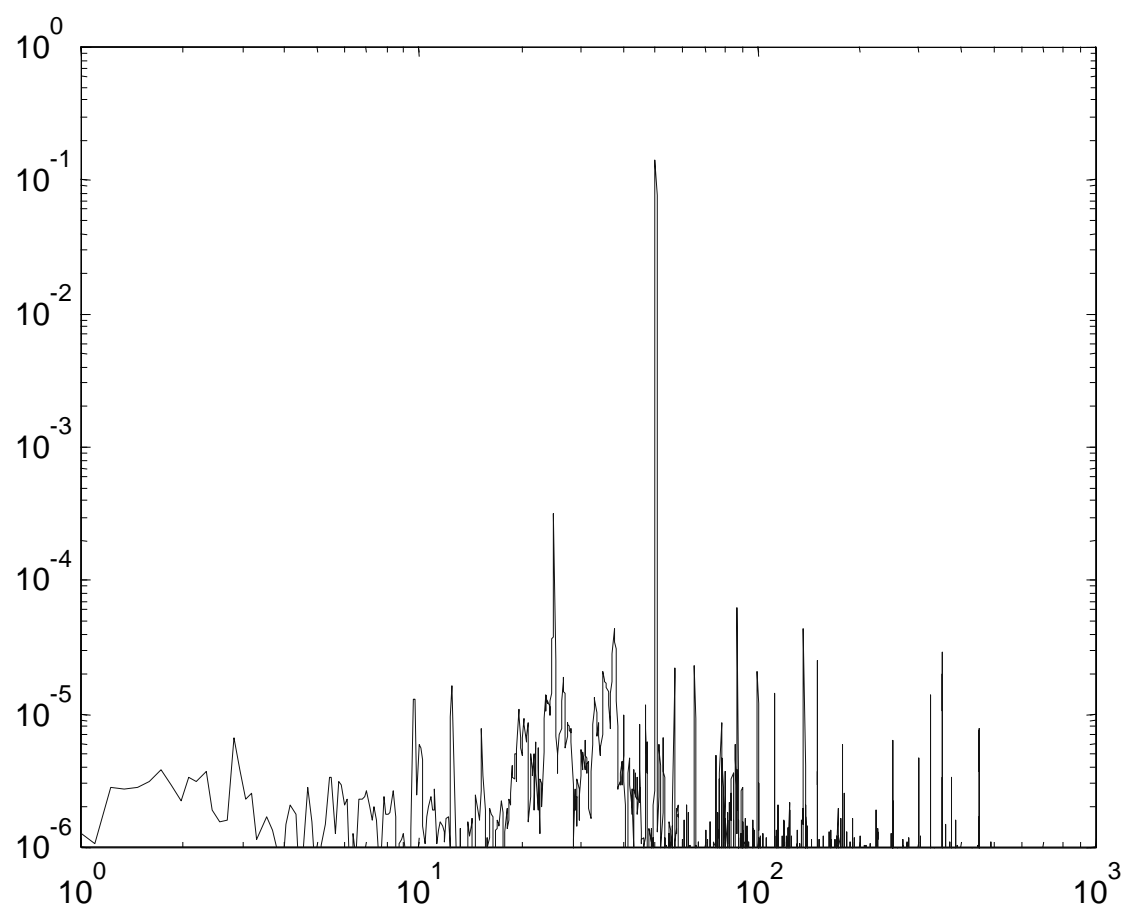

Figura 4.3: Ejemplo de espectro de una señal.

La Figura 4.3 muestra un espectro de una señal de ejemplo, con distribuciones de energía entre 1 y $3 \mathrm{~Hz}$, entre 2 y $50 \mathrm{~Hz}$, y concentrada en un pico a $50 \mathrm{~Hz}$ y picos aislados a otras frecuencias.

El cálculo de la transformada de Fourier para señales discretas de $2^{N}$ valores instantáneos se realiza computacionalmente con el algoritmo conocido como FFT o "Fast Fourier Transform".

El cálculo de la distribución espectral de una señal presenta algunas dificultades:

- En principio, el desvío standard del valor calculado para una frecuencia dada, medida de su dispersión, o incertidumbre, es tan grande como el mismo valor calculado [23], es decir:

$$
\frac{\sigma_{\hat{x}}(n)}{\hat{x}(n)} \approx 1
$$




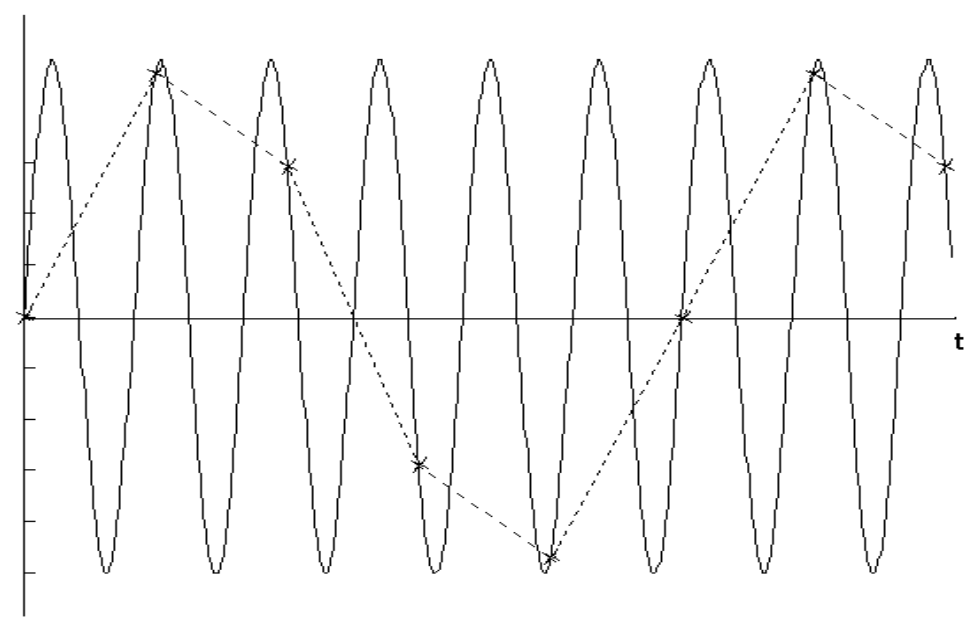

Figura 4.4: Efecto del muestreo a una frecuencia inferior a la frecuencia de Nyquist.

La incertidumbre se reduce dividiendo la señal en $N s$ segmentos o "bloques" y promediando los espectros de los $N s$ bloques. En este caso, el desvío standard se reduce en un factor $\frac{1}{\sqrt{N s}}$.

- Para una señal adquirida con una frecuencia de muestreo $f_{m}$, la máxima frecuencia del espectro que puede interpretarse en forma unívoca es la frecuencia de Nyquist, $f_{m} / 2$. La señal debe ser procesada con un filtro pasa-bajo que elimine frecuencias más altas, para evitar el fenómeno de "aliasing" o aparición de falsos valores de espectro en bajas frecuencias. La Figura 4.4 muestra este efecto: al muestrear (marcas x) una señal (línea continua) con una frecuencia de muestreo muy baja, se genera un "alias" o señal fantasma de baja frecuencia (línea punteada), inexistente en la señal original, que distorsiona el espectro real.

- La resolución del espectro está dada por $\Delta f=\frac{1}{T}=\frac{1}{N \Delta t}$. Una vez especificada la frecuencia de muestreo $1 / \Delta t$, la resolución del espectro depende del tamaño del registro, $N$. Si se esperan picos de ancho de banda pequeños, $N$ debe ser lo suficientemente grande para que $\Delta f=\frac{1}{N \Delta t}$ pueda captarlos con buena resolución.

- Cuando se utiliza una muestra finita de una señal, durante un tiempo $T$, implícitamente se involucra una ventana rectangular que multiplica a la señal en el dominio del tiempo. La muestra finita es matemáticamente el equivalente a 
multiplicar una señal de duración infinita por una función ("ventana") $w(t)$ que vale 1 entre 0 y $T$ y 0 para los demás valores de $t$. Ello significa, por propiedades de la transformación de Fourier, que en el dominio de la frecuencia se produce la convolución entre el espectro de la señal y el espectro de la ventana rectangular (ver p. ej. Schwartz and Shaw [24]). Dicha convolución produce dispersión de la energía de la señal en componentes adyacentes de frecuencia debido a la presencia de lóbulos laterales en el espectro de la ventana rectangular (Figura 4.5). Debido a esta dispersión, la amplitud de las componentes senoidales que se desee examinar difiere de su valor real. Para reducir la interferencia de estas frecuencias, se utilizan otras funciones "ventana". Una de uso extendido, utilizada en este trabajo, es la llamada ventana de Hanning:

$$
w_{H}(t)=\left\{\begin{array}{l}
\frac{1}{2}\left(1-\cos \frac{2 \pi t}{T}\right)=1-\cos ^{2} \frac{\pi t}{T} \quad 0 \leq t \leq T \\
0 \quad \text { en otro caso }
\end{array}\right.
$$

Los lóbulos laterales del espectro de esta ventana son de menor amplitud que los de la ventana rectangular. Sin embargo, su lóbulo principal es mayor, de forma que se reduce la resolución de la FFT (Figura 4.6). En la elección de la ventana existe entonces un compromiso entre la resolución requerida y el grado de distorsión permitido.
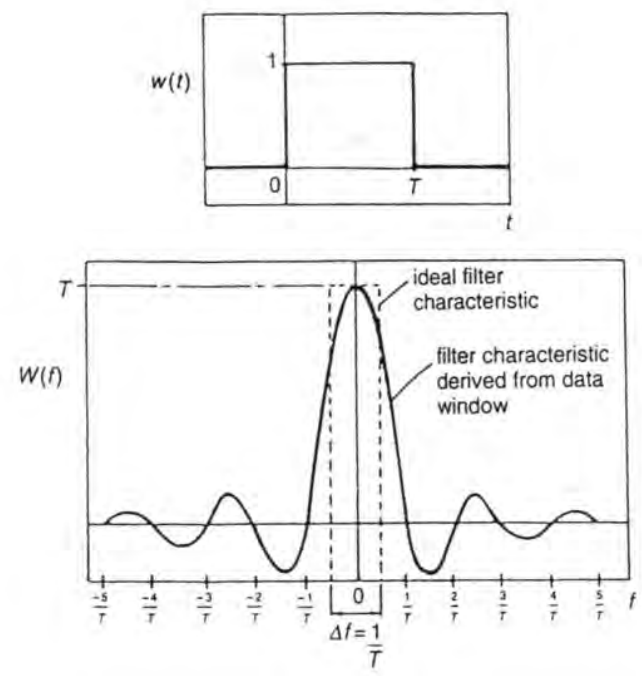


\section{CAPÍTULO 4. PROCESAMIENTO DE DATOS}

Figura 4.5: Ventana rectangular implícita al analizar un intervalo finito $(0, T)$.
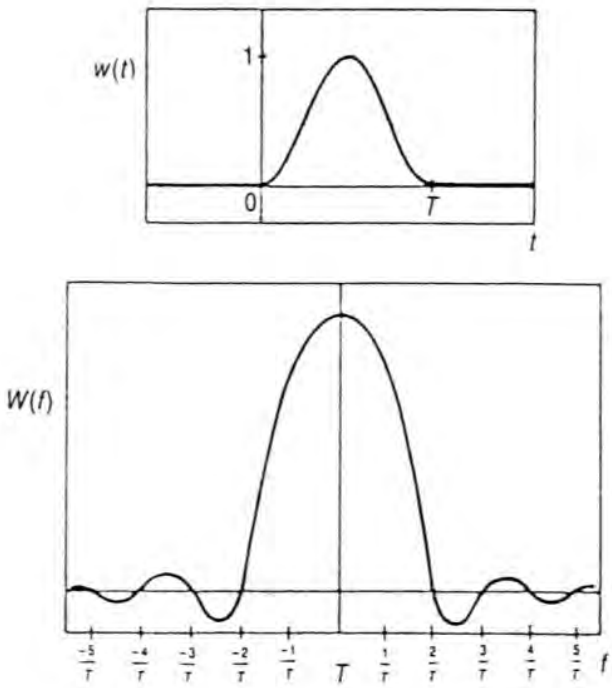

Figura 4.6: Ventana Hanning implícita al analizar un intervalo finito $(0, T)$. 


\section{CAPÍtulo 5}

\section{ANÁlisis de Resultados}

En este apartado se analizan los resultados correspondientes al procesamiento de datos provenientes de las señales adquiridas mediante anemometría de hilo caliente. De acuerdo a la planificación de los ensayos se midieron los perfiles de velocidades en función de la altura (eje y) y la posición aguas abajo para diferentes casos. Si bien se cuenta con una gran cantidad de información proveniente de los experimentos, se decidió estudiar solo una condición de ensayo como ser 0 grados de ángulo de ataque y una velocidad de la corriente libre de $10 \mathrm{~m} / \mathrm{s}$ a modo de no entorpecer el análisis. Se utilizaron tres modelos con el mismo perfil base y diferentes dispositivos de control activo de flujo como ya se describió anteriormente. En esta etapa, se busca adimensionalizar las variables para poder comparar los resultados de los diferentes modelos. Se realizó un análisis detallado de las curvas como así también de las tablas con parámetros de interés obtenidas del procesamiento de datos para cada modelo y una comparación final a modo de estudiar el comportamiento de dichos dispositivos. Algunos resultados de estos estudios se reportaron en los abstracts y resúmenes que se detallan en las referencias.

\subsection{Perfil de Velocidades Medias}

\subsubsection{Componente $u$}

\subsubsection{Caso Mini-flap Gurney Rotante}

Analizando las curvas de la Figura 5.1, se observa que el mínimo del perfil de velocidades para el caso sin mini-flap Gurney se da a la altura del borde de fuga, es decir en el punto de estagnación posterior. No es así el caso del perfil con mini-flap Gurney fijo que se da a aproximadamente $2 \%$ c por debajo del borde de fuga. También se ve que la región de mínima velocidad es más ancha que en el caso anterior. Si se observan los casos con frecuencia de activación $(\mathrm{F} 1=22 \mathrm{~Hz}, \mathrm{~F} 2=38 \mathrm{~Hz}$ y $\mathrm{F} 3=44 \mathrm{~Hz})$, los mínimos están un poco por debajo del borde de fuga y los valores son de similar magnitud que el caso del perfil sin mini-flap Gurney pero con una dispersión intermedia a los casos anteriores. 
Aguas abajo (Figura 5.2), se observa el mismo comportamiento pero ya la posición de los mínimos se da en todos los casos por debajo del borde de fuga. Al mismo tiempo la pérdida de cantidad de movimiento del flujo es mucho mayor con el mini-flap Gurney en cualquiera de sus configuraciones que para el perfil limpio. Es evidente que la resistencia aerodinámica del perfil aumenta, aunque esto se ve compensado por el mayor coeficiente de sustentación, brindando en conjunto una mayor eficiencia [25]. Si pensamos que el perfil se encuentra en una condición de sustentación con un ángulo de ataque $\left(0^{\circ}\right)$ mayor al de sustentación nula, existirá una componente vertical de velocidad (downwash) que desplaza hacia abajo el perfil de velocidades de la componente $u$. Este fenómeno se verá reflejado en la componente $v$ más adelante. También para el caso del perfil sin mini-flap Gurney se observa que el mínimo de velocidad es mucho más pronunciado que en el resto de los casos. Esto se podría explicar desde el punto de vista de la intensidad de turbulencia que introducen dichos mecanismos aumentando la mezcla de las capas exteriores. Se observa también que las curvas de velocidad correspondientes a los casos de Gurney activos son más suaves que el resto de los casos con un comportamiento similar al caso del $2 \%$ c [29].

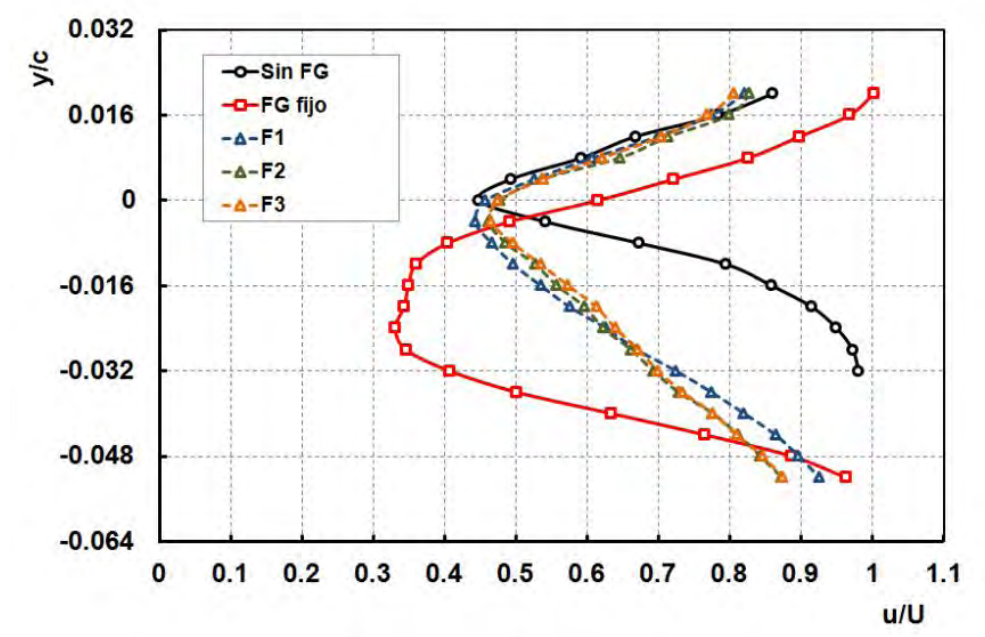

Figura 5.1: Perfil de velocidades medias "u" al 2\%c - Mini-flap Rotante. 


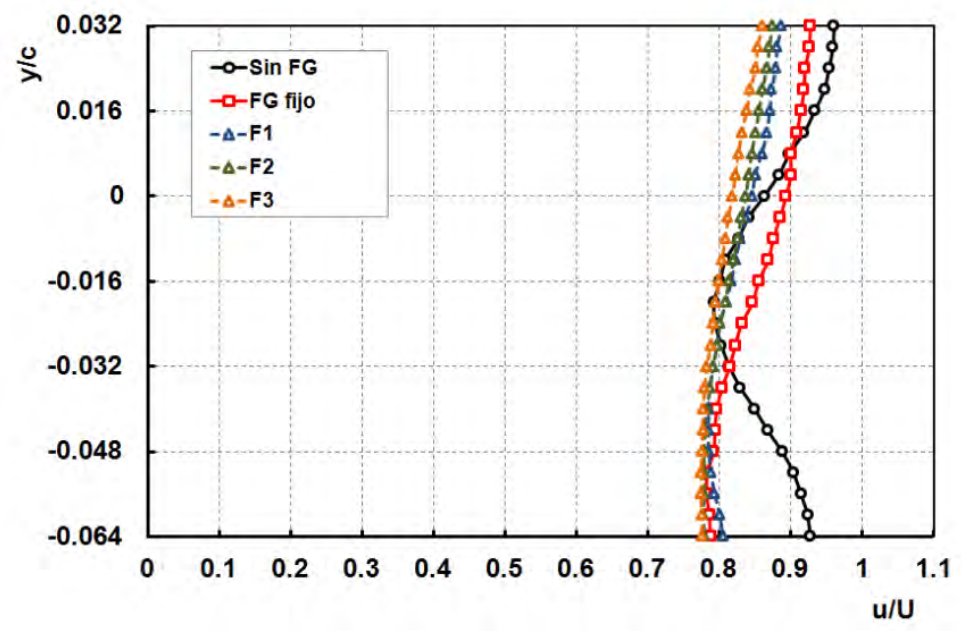

Figura 5.2: Perfil de velocidades medias "u" al 75\%c - Mini-flap Rotante.

\subsubsection{Caso Mini-flap Gurney Rotante (Variante $30^{\circ}$ )}

Observando los perfiles de velocidades (Figuras 5.3, 5.4 y 5.5) se ve un mínimo definido para el caso del perfil sin mini-flap Gurney a la altura del borde de fuga. Para el caso del mini-flap Gurney fijo se da que el mínimo es más intenso y se ubica más por debajo del caso sin mini-flap Gurney.

Los valores para los caso de las curvas correspondientes a frecuencia se encuentran entre los valores correspondientes a los casos de perfil sin y con mini-flap Gurney fijo. Aquí también se observa que a medida que me alejo aguas abajo el mínimo de velocidad se desplaza hacia abajo como consecuencia del "downwash". También se observa que los mínimos de velocidad se reducen y se ensanchan aguas abajo debido a la mezcla de las diferentes capas.

Las frecuencias F2 y F3 producen efectos similares sobre el flujo, introduciendo un descenso moderado del mínimo. La frecuencia F1, por otra parte, aumenta el descenso del mínimo de velocidad y reduce la pérdida de cantidad de movimiento a mayor distancia $(10 \%$ c), por lo que aparece como una buena combinación para el perfil. Este comportamiento se verifica también con los resultados para la componente vertical $v$ [33]. 


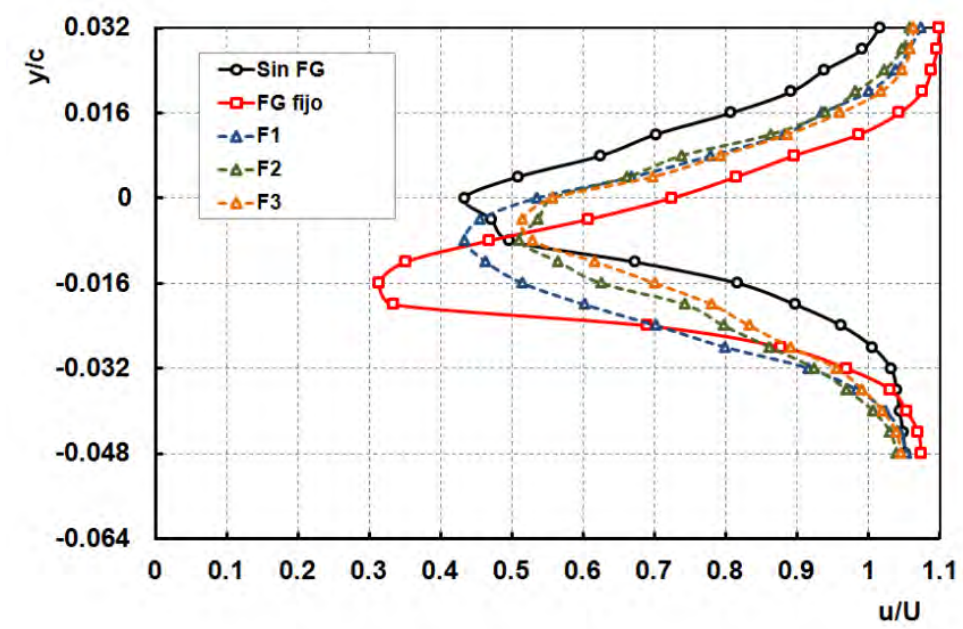

Figura 5.3: Perfil de velocidades medias "u" al 2\%c - Mini-flap Rotante $\left(30^{\circ}\right)$.

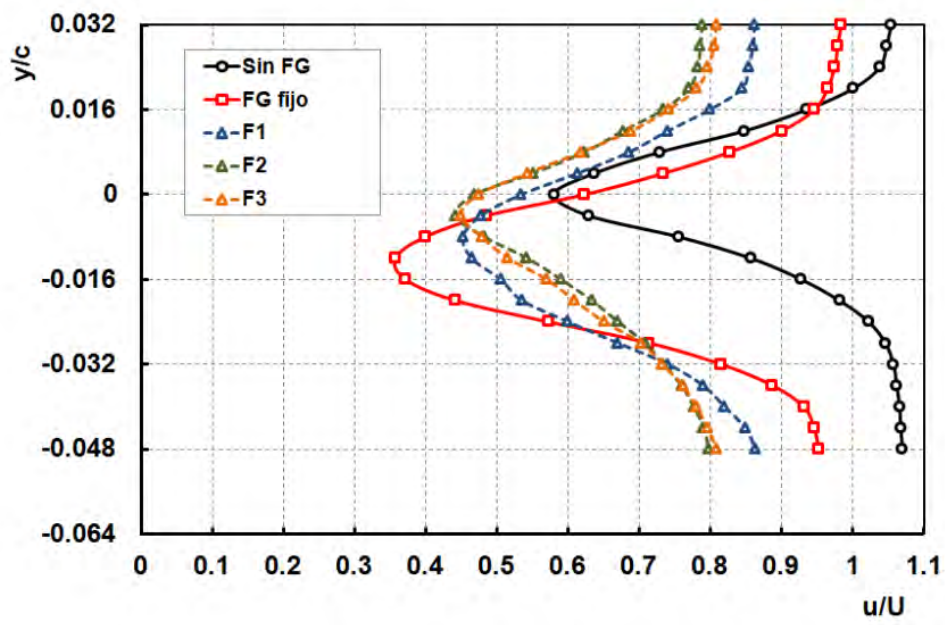

Figura 5.4: Perfil de velocidades medias "u" al 4\%c - Mini-flap Rotante $\left(30^{\circ}\right)$.

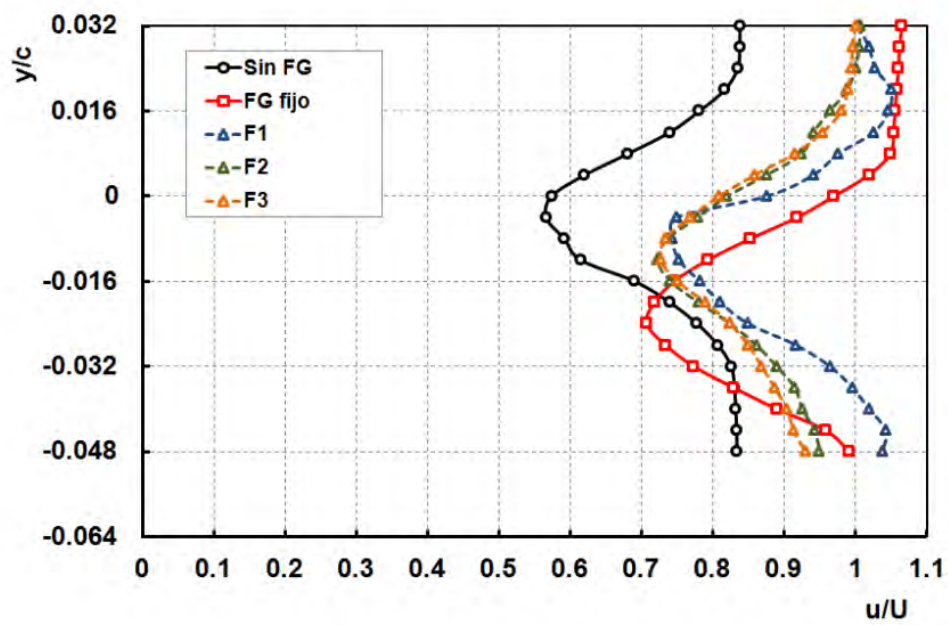

Figura 5.5: Perfil de velocidades medias "u" al 10\%c - Mini-flap Rotante (30). 


\subsubsection{Caso Mini-flap Gurney Móvil}

Para este caso (Figuras 5.6, 5.7 y 5.8), los valores de los mínimos se dan a la altura del borde de fuga para los casos de perfil sin mini-flap Gurney y para los casos de frecuencia. En cambio en el caso del mini-flap Gurney fijo el mínimo se da más abajo. Se observa también que el perfil es más ancho en el mismo caso respecto del resto. Los mínimos de las curvas correspondientes a los casos con frecuencia son menores que el resto de los casos, con lo que si pensamos que el perfil de velocidades detrás del perfil nos da una idea de la pérdida de cantidad de movimiento en el fluido, podríamos concluir acerca de la variación de la resistencia del perfil con diferentes mecanismos. Este comportamiento se mantiene a medida que nos movemos aguas abajo. Al mismo tiempo que nos alejamos del borde de fuga los valores de los mínimos se reducen y se dispersan como era de esperar. Los resultados para esta configuración de mini-flap no muestran mejoras evidentes en la performance del perfil.

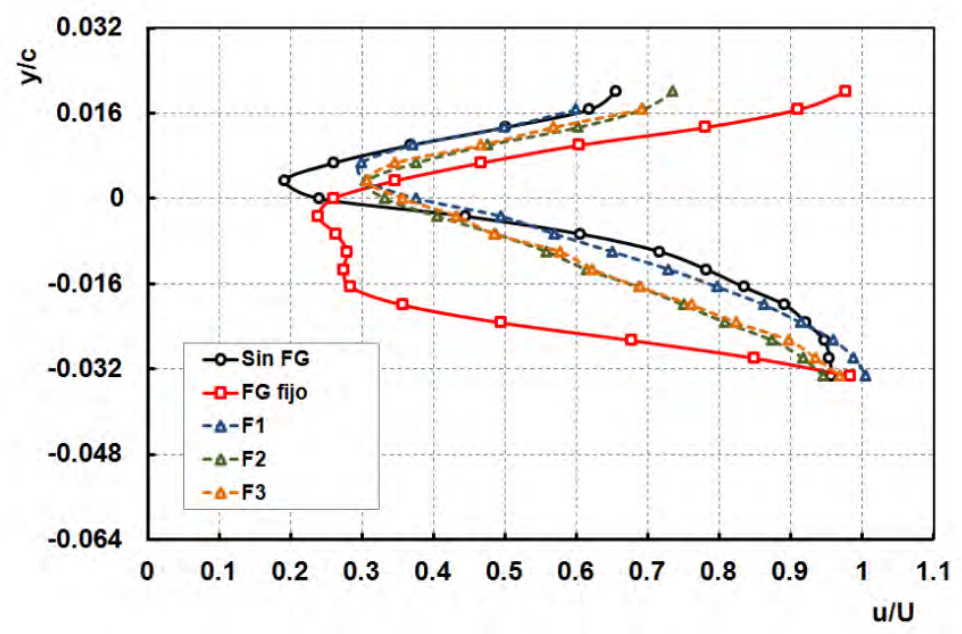

Figura 5.6: Perfil de velocidades medias "u" al 2\%c - Mini-flap Móvil.

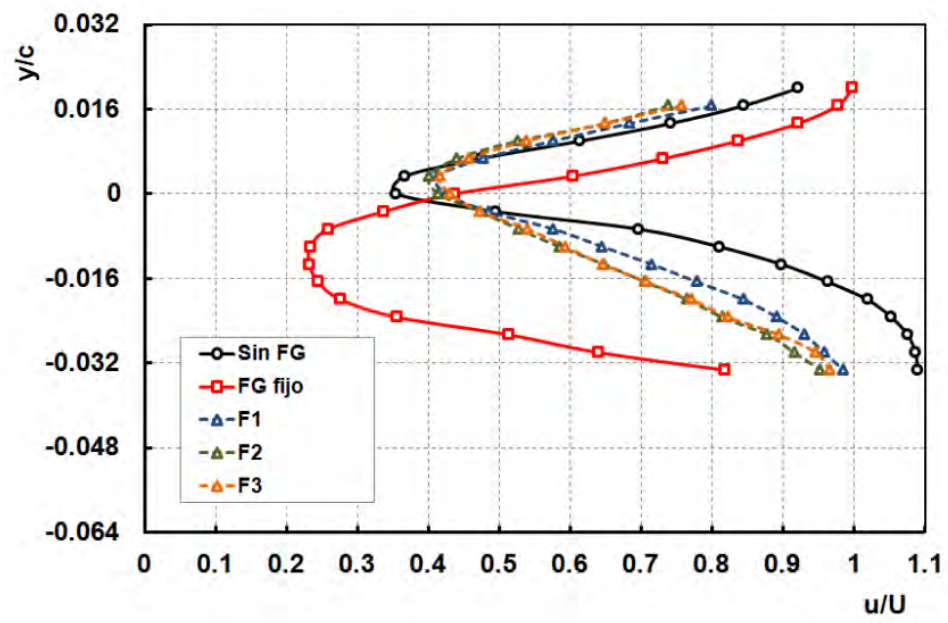

Figura 5.7: Perfil de velocidades medias "u" al 4\%c - Mini-flap Móvil. 


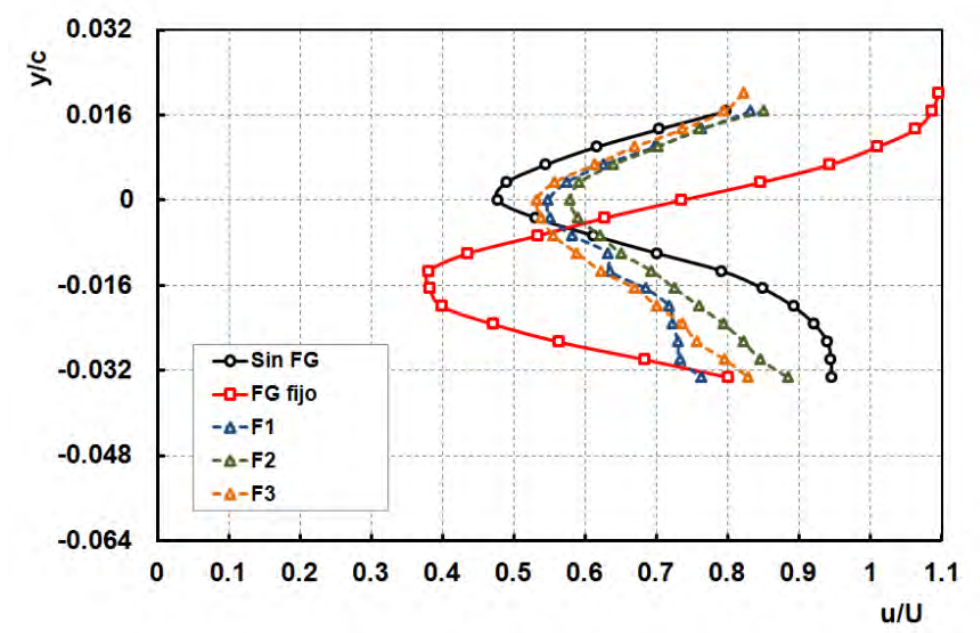

Figura 5.8: Perfil de velocidades medias "u" al 10\%c - Mini-flap Móvil.

\subsubsection{Comparación de Dispositivos - Velocidad Media $u$}

Comparando las gráficas de los diferentes dispositivos al $2 \%$ c se observa que los mínimos de velocidad para el caso del perfil sin mini-flap Gurney se dan a la altura del borde de fuga. Aquí la región de baja velocidad es más angosta que en el resto de los casos. Para los diferentes dispositivos se ve que el mini-flap Gurney fijo es el que produce mayores reducciones en la velocidad y por lo tanto un aumento en el coeficiente de resistencia. También se ve que las curvas en estos casos se extienden en un rango mayor de alturas.

Cuando se analizan los casos con frecuencia, se ve que los mínimos son de menor intensidad que los casos sin y con mini-flap Gurney, pero las curvas manifiestan un mínimo a la altura del borde de fuga similar al perfil solo y luego se hacen más anchas como el caso del perfil con mini-flap Gurney. Esto se observa en la mayoría de las curvas. La mayor reducción de velocidad detrás del borde de fuga se logra con el dispositivo móvil. Es difícil establecer una tendencia respecto de los casos con frecuencia de activación ya que las variaciones no son mayores, pero en la mayoría de las curvas se da que con la frecuencia 1 se alcanza el mínimo más importante. Un fenómeno esperable es el aumento de la velocidad a medida que me alejo aguas abajo.

\subsubsection{Componente $V$}

\subsubsection{Caso Mini-flap Gurney Rotante}

Para el caso del perfil sin mini-flap Gurney se observa que no hay valores positivos en el perfil de velocidades mientras que en la zona superior con fluido proveniente del extradós se observan valores negativos lo que nos da un indicio de una corriente descendente (downwash) como ya se había mencionado en el caso del análisis de la componente longitudinal de velocidad $u$ (Figura 5.9). No es así en el resto de los casos, es 
decir, mini-flap Gurney fijo y con frecuencia, ya que en la zona inferior se ve una componente hacia arriba de velocidad.

Aguas abajo (Figura 5.10), los perfiles de velocidad son aproximadamente planos pero diferentes de cero lo que da una idea de que a esa altura el flujo tienen una componente descendente producto de la sustentación del perfil. También se ve que la mayor componente de "downwash" se da para el caso del perfil con mini-flap Gurney fijo siguiendo por los casos con frecuencia y el perfil sin mini-flap Gurney. Esto nos puede dar una idea de que el Gurney efectivamente aumenta la circulación promedio alrededor del perfil de acuerdo a lo que describen otros autores en sus experimentos con mini-flaps Gurney.

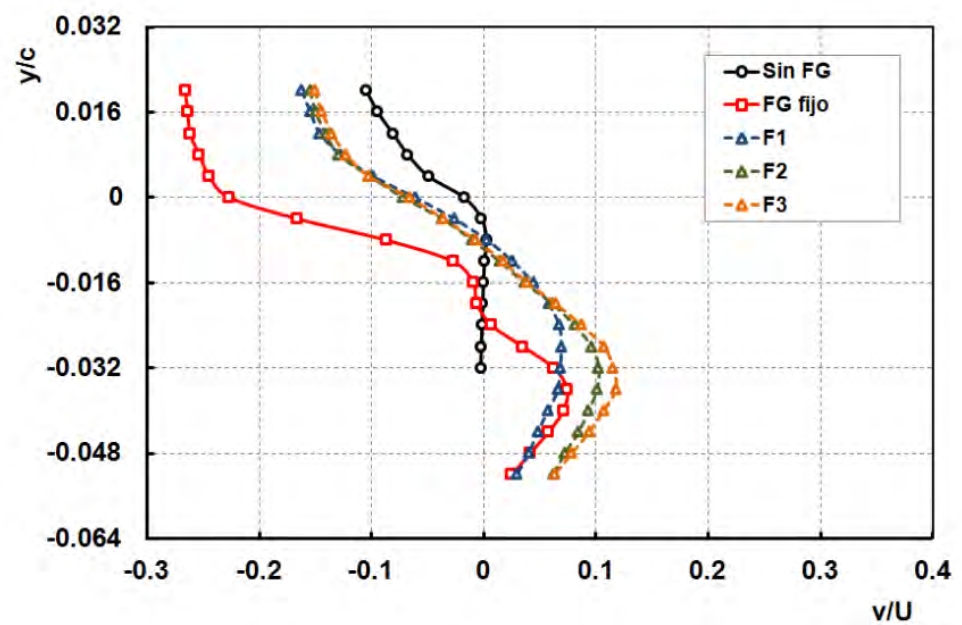

Figura 5.9: Perfil de velocidades medias "v" al 2\%c - Mini-flap Rotante.

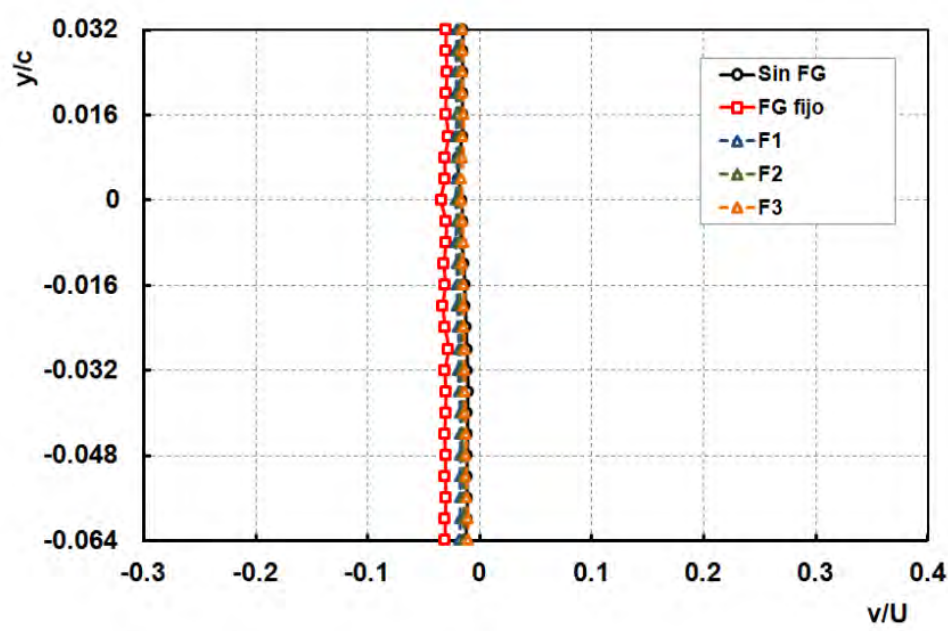

Figura 5.10: Perfil de velocidades medias "v" al 75\%c - Mini-flap Rotante. 


\subsubsection{Caso Mini-flap Gurney Rotante (Variante $30^{\circ}$ )}

En el perfil sin mini-flap Gurney se observa (Figura 5.11) por debajo del borde de fuga una pequeña componente hacia arriba que se vuelve negativa a medida que me alejo aguas abajo. En cambio en la zona superior con fluido proveniente del extradós, las componentes verticales de velocidad tienen un valor negativo lo que da una idea de la existencia nuevamente de "downwash" propio de la circulación del perfil con ángulos de ataque superiores al de sustentación nula. Cerca del borde de fuga del mini-flap Gurney se observa un mínimo tanto en el caso fijo como en los casos con frecuencia, más acentuado en el primero. A medida que me alejo aguas abajo (Figuras 5.12 y 5.13) estos mínimos se dispersan, disminuyen su intensidad y hasta cambian de signo producto de la difusión y la predominancia de la corriente descendente. Para los casos con frecuencia se ve que los perfiles de velocidad son más suaves. También se observa que al 10\%c (Figura 5.13) aguas abajo las componentes de velocidad vertical son mayores en el caso del minimini-flap Gurney fijo que en el resto de los casos por lo que nos da una idea de que la sustentación es mayor.

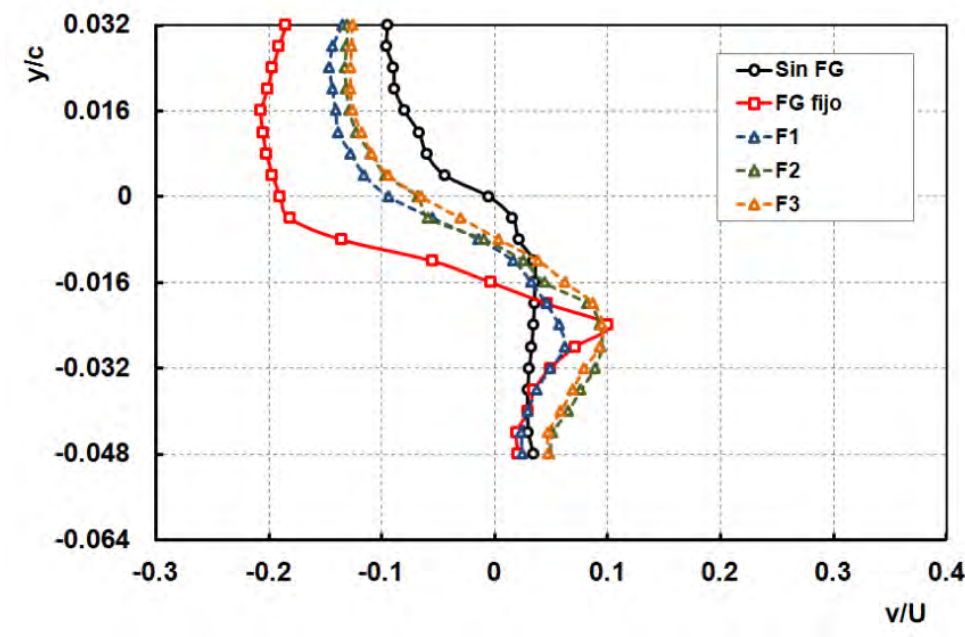

Figura 5.11: Perfil de velocidades medias "v" al 2\%c - Mini-flap Rotante $\left(30^{\circ}\right)$.

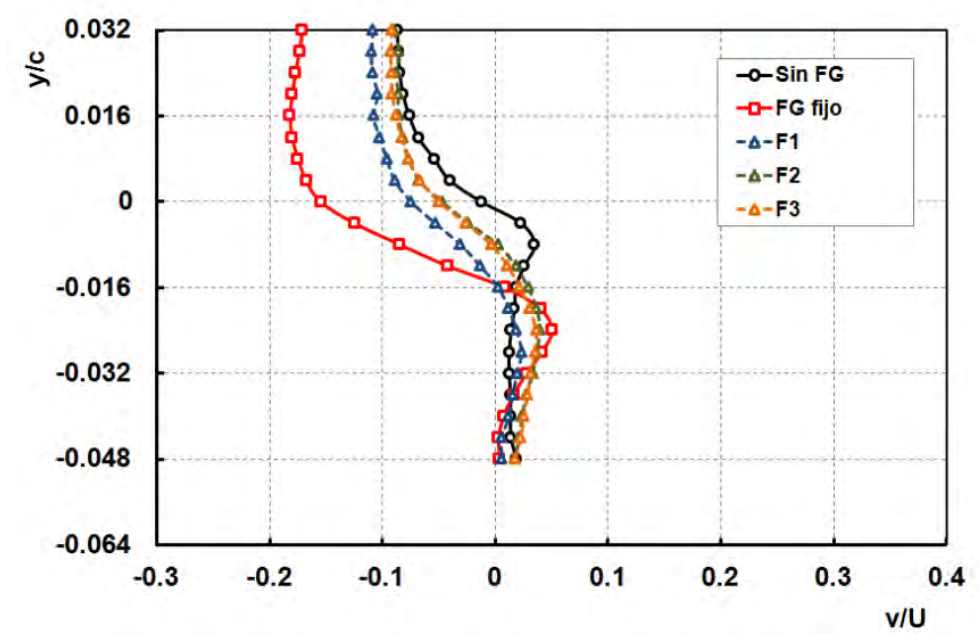

Figura 5.12: Perfil de velocidades medias "v" al 4\%c - Mini-flap Rotante (30). 


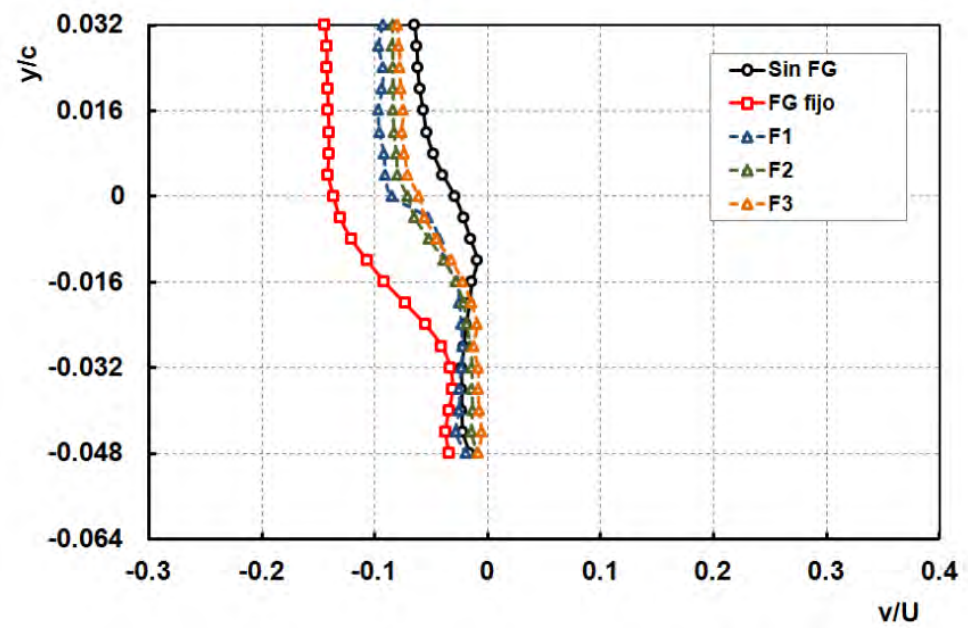

Figura 5.13: Perfil de velocidades medias "v" al 10\%c - Mini-flap Rotante $\left(30^{\circ}\right)$.

\subsubsection{Caso Mini-flap Gurney Móvil}

Si analizamos el perfil sin mini-flap Gurney arriba del borde de fuga en la Figura 5.14 observamos nuevamente la presencia de "downwash", mientras que en la zona inferior se dan componentes en sentido opuesto. Este efecto se reduce y se invierte aguas abajo (Figuras 5.15 y 5.16).

En las tres gráficas se ve que el efecto de la componente vertical de velocidad proveniente del extradós es mucho mayor en el caso del mini-flap Gurney fijo respecto del resto de los casos. En los casos con frecuencia, los valores se dan entre medio de los casos de perfil sin y con mini-flap Gurney fijo siendo los valores mínimos más suaves.

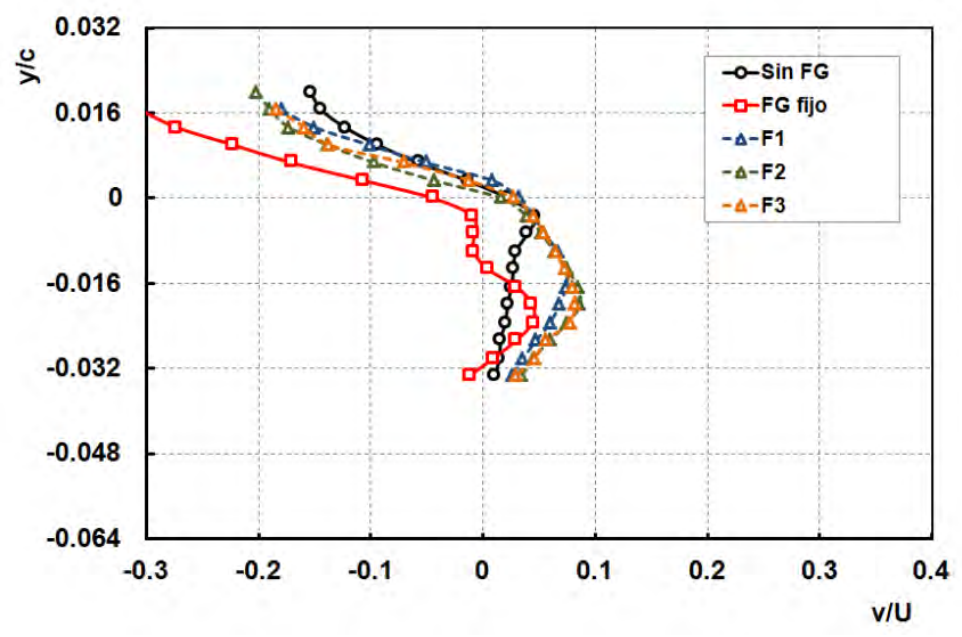

Figura 5.14: Perfil de velocidades medias "v" al 2\%c - Mini-flap Móvil. 


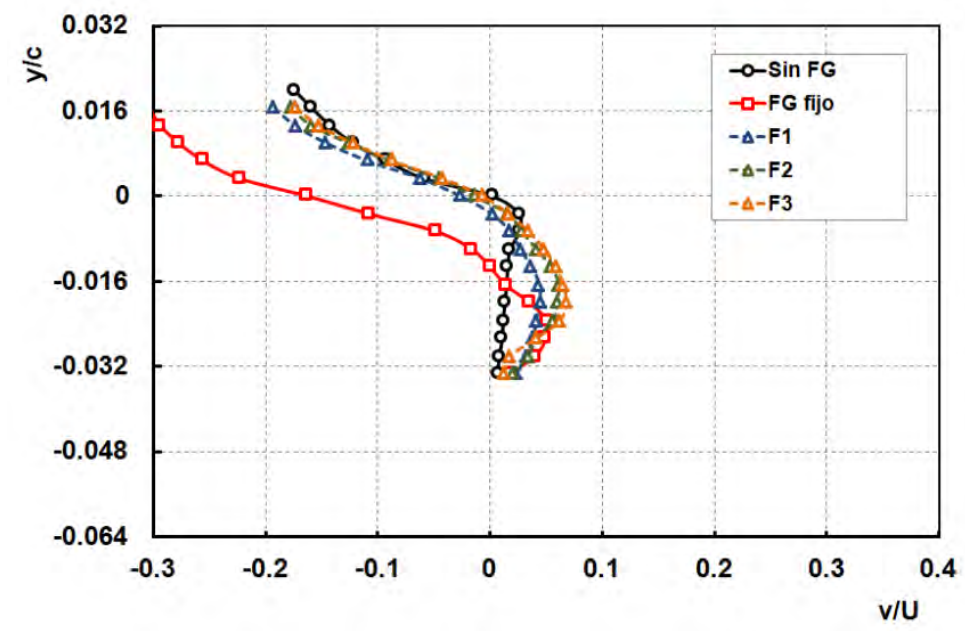

Figura 5.15: Perfil de velocidades medias "v" al 4\%c - Mini-flap Móvil.

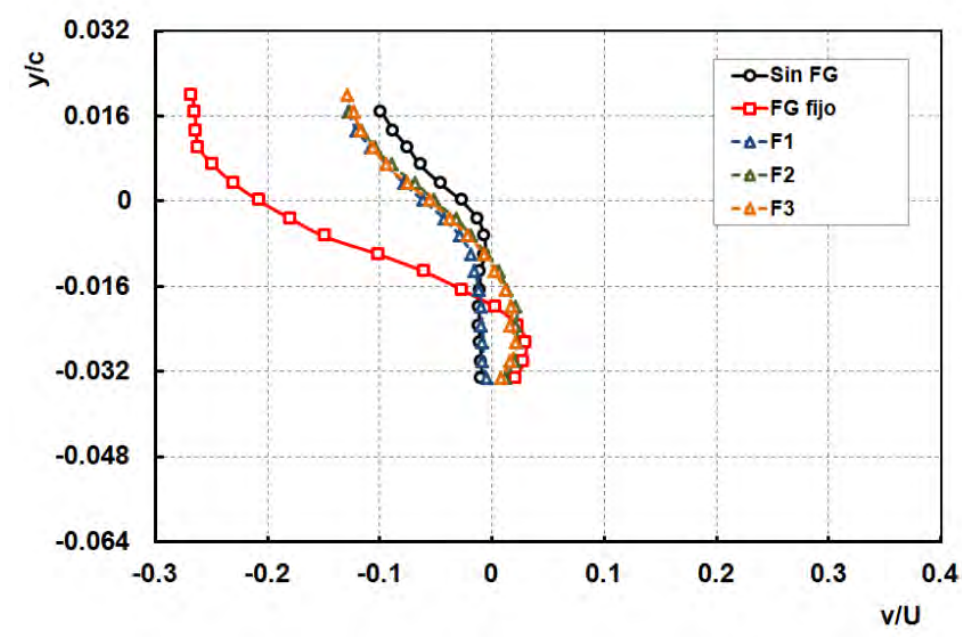

Figura 5.16: Perfil de velocidades medias "v" al 10\%c - Mini-flap Móvil.

\subsubsection{Comparación de Dispositivos - Velocidad Media $v$}

Si comparamos los diferentes dispositivos teniendo en cuenta los perfiles de velocidad media de la componente vertical $v$ vemos que en todos los casos existe una componente descendente en la zona superior del borde de fuga con fluido proveniente del extradós. En cambio en la zona inferior se observa una componente vertical ascendente. Dichas componentes se acentúan dependiendo del dispositivo utilizado y de la frecuencia de activación. En cambio, si nos desplazamos aguas abajo, dichos perfiles de velocidad disminuyen su intensidad.

Los mayores niveles de velocidad se dan para el caso de perfil con mini-flap Gurney en todos los casos, mientras que los menores se dan para el perfil sin dispositivos. Si analizamos los casos con frecuencia vemos que se comportan en forma intermedia entre 
los casos con y sin mini-flap Gurney fijo. Existe nuevamente una tendencia al modificar la frecuencia de activación de los mecanismos. A mayor frecuencia entonces aumentan negativamente los niveles de la velocidad vertical.

Cabe mencionar que en el caso de mini-flap móvil los valores de la velocidad vertical se mantienen aproximadamente constantes aguas abajo respecto de los mecanismos rotantes. También hay que mencionar que las curvas se desplazan en el eje de altura dependiendo de los dispositivos utilizados lo que no puede llegar a dar una idea de la modificación del punto de estagnación posterior. Este comportamiento también está presente en las curvas del perfil de velocidades de la componente horizontal [25, 28, 30].

\subsection{Perfil de Intensidad de Turbulencia}

\subsubsection{Componente $u$}

\subsubsection{Caso Mini-flap Gurney Rotante}

Analizando las curvas de la Figura 5.17 se ve que los niveles de intensidad de turbulencia son menores en el caso del perfil sin mini-flap Gurney que en el resto de los casos. Se observa para este caso también un mínimo en la cresta de la curva. La mayor intensidad de turbulencia se da para el caso del perfil con mini-flap Gurney fijo y a la atura del borde de fuga del mismo siendo más extensa y constante en un rango de alturas respecto del resto de los casos.

Las curvas de intensidades de turbulencia correspondientes a frecuencia son un caso intermedio, es decir, los niveles de intensidad son similares al caso de mini-flap Gurney fijo pero ubicados más cerca de la altura del borde de fuga. Estudiando solamente los casos activos, la frecuencia 1 produce niveles de turbulencia similares al producido por el mini-flap Gurney fijo y mayores que las frecuencias 2 y 3.

Aguas abajo (Figura 5.18), el comportamiento es similar respecto del 2\%c detrás del borde de fuga. La menor intensidad se da para el caso del perfil sin mini-flap Gurney. Luego, los valores para el resto de los casos son de similar intensidad pero a diferentes alturas y con diferente dispersión, es decir más suave para los casos con frecuencia. También se puede deducir que a medida que la frecuencia de activación del dispositivo aumenta la intensidad de turbulencia disminuye. Se observa que los valores de intensidad de turbulencia son mucho menores que al $2 \%$ c detrás del borde de fuga para todos los casos. 


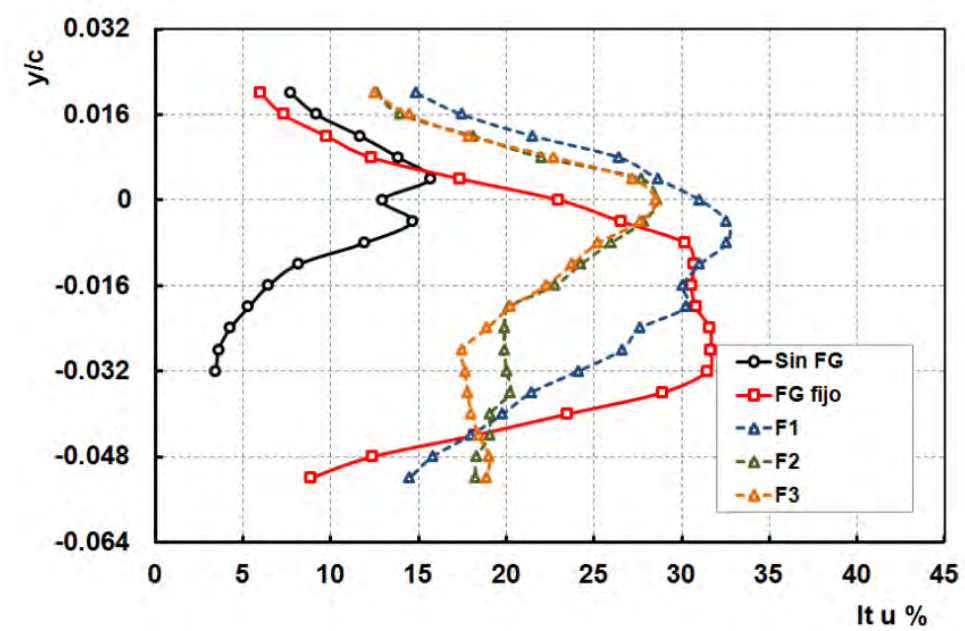

Figura 5.17: Perfil de intensidad de turbulencia "u" al 2\%c - Mini-flap Rotante.

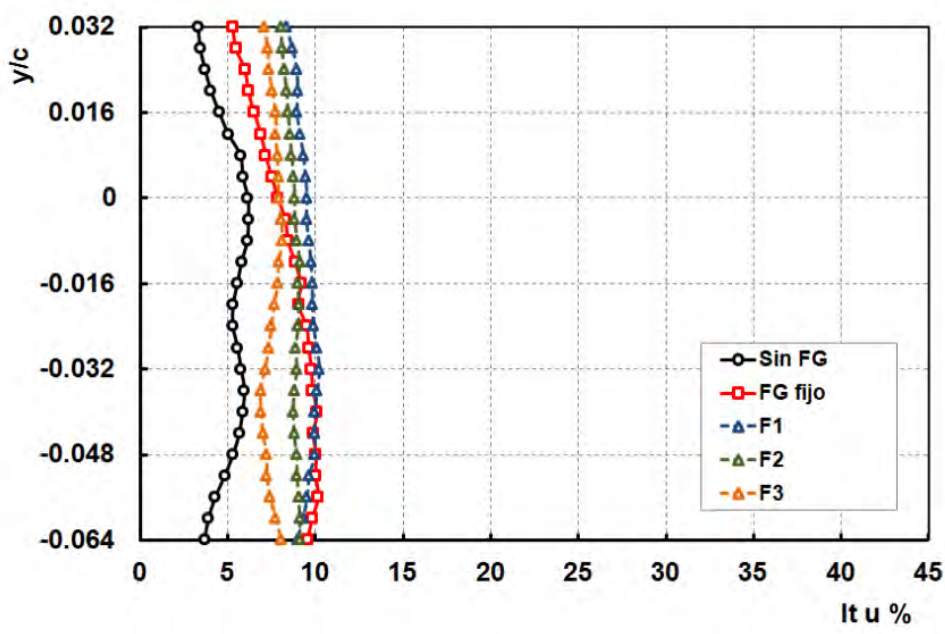

Figura 5.18: Perfil de intensidad de turbulencia "u" al 75\%c - Mini-flap Rotante.

\subsubsection{Caso Mini-flap Gurney Rotante (Variante $30^{\circ}$ )}

$\mathrm{Al}$ igual que en el caso anterior, al 2\%c (Figura 5.19) el perfil sin mini-flap Gurney tiene menor intensidad de turbulencia que el resto. Se observa también un mínimo a la altura del borde de fuga del mini-flap Gurney. Es de destacar en este dispositivo que el pico de mayor intensidad se da para el caso de dispositivo activo con frecuencia 1, aun mayor que el caso de mini-flap Gurney fijo. Este comportamiento se da en las tres gráficas de intensidad de turbulencia aguas abajo (2\%c, 4\% y 10\%c). Para el resto de las frecuencias de activación, los valores de turbulencia tienen niveles intermedios.

Aguas abajo (Figuras 5.21 y 5.22) el comportamiento es similar al 2\%c, es decir, se observa el pico de intensidad para el caso de frecuencia 1. Los valores de intensidad de turbulencia decaen a medida que me alejo en la estela. Aquí también está presente el mínimo en la cresta del pico de las intensidades de turbulencia. 


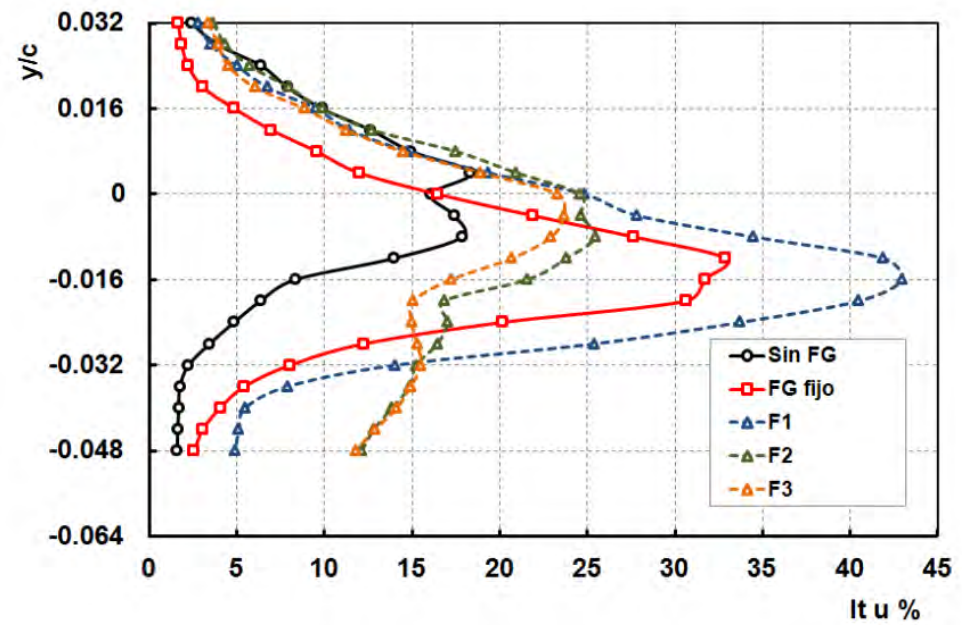

Figura 5.19: Perfil de intensidad de turbulencia "u" al 2\%c - Mini-flap Rotante $\left(30^{\circ}\right)$.

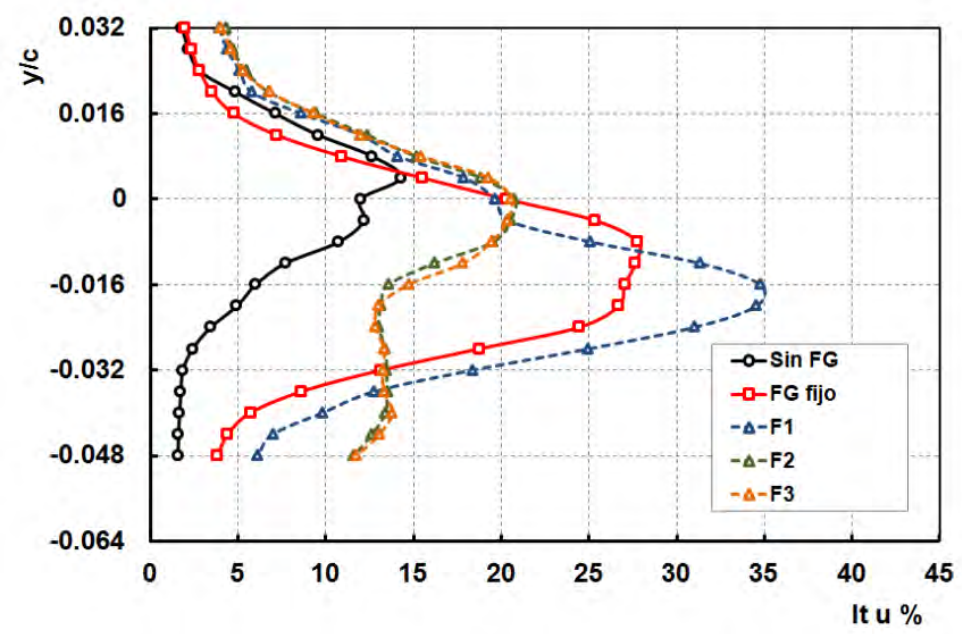

Figura 5.20: Perfil de intensidad de turbulencia "u" al 4\%c - Mini-flap Rotante $\left(30^{\circ}\right)$.

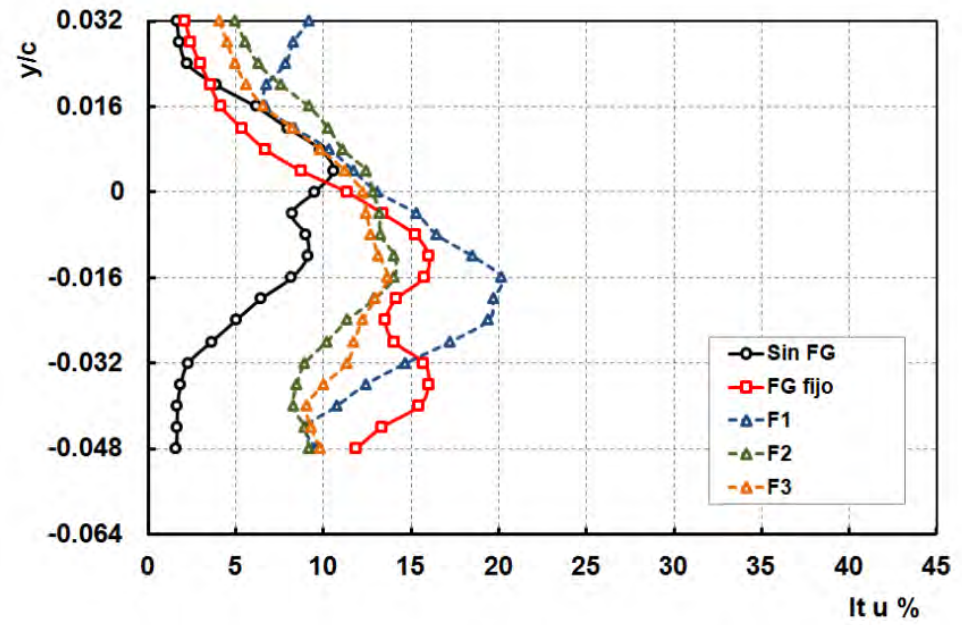

Figura 5.21: Perfil de intensidad de turbulencia "u" al 10\%c - Mini-flap Rotante $\left(30^{\circ}\right)$. 


\subsubsection{Caso Mini-flap Gurney Móvil}

Para este dispositivo, la menor intensidad de turbulencia se da para el caso del perfil sin mini-flap Gurney y a la altura del borde de fuga. En cambio el caso de mayor intensidad se da en el perfil con mini-flap Gurney fijo (Figuras 5.22, 5.23 y 5.24).

Cuando se analizan los casos con activación de frecuencia se da que los picos de intensidad son de similar orden de magnitud que en el caso del mini-flap Gurney fijo y aún mayores como es el caso de la frecuencia 1. También se da en los casos con frecuencia que los mínimos son tan concentrados como en el caso del perfil solo. No es así en el caso del mini-flap Gurney fijo donde el pico es más ancho. Los valores de intensidad se vuelven menores y más suaves a medida que me alejo del borde de fuga para los casos con frecuencia y perfil solo, pero para el caso del mini-flap Gurney fijo se mantienen sus niveles de intensidad y anchura aproximadamente constantes. En este dispositivo se ve que los valores de máxima intensidad se dan a la altura del borde de fuga para los casos de frecuencia y perfil sin mini-flap Gurney. Dicha ubicación se mantiene aguas abajo.

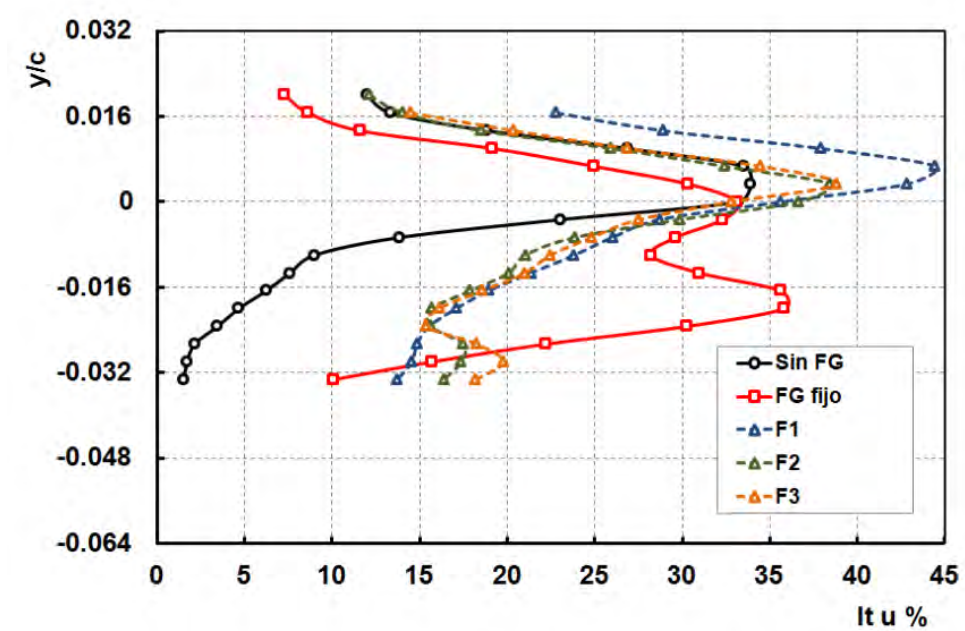

Figura 5.22: Perfil de intensidad de turbulencia "u" al 2\%c - Mini-flap Móvil. 


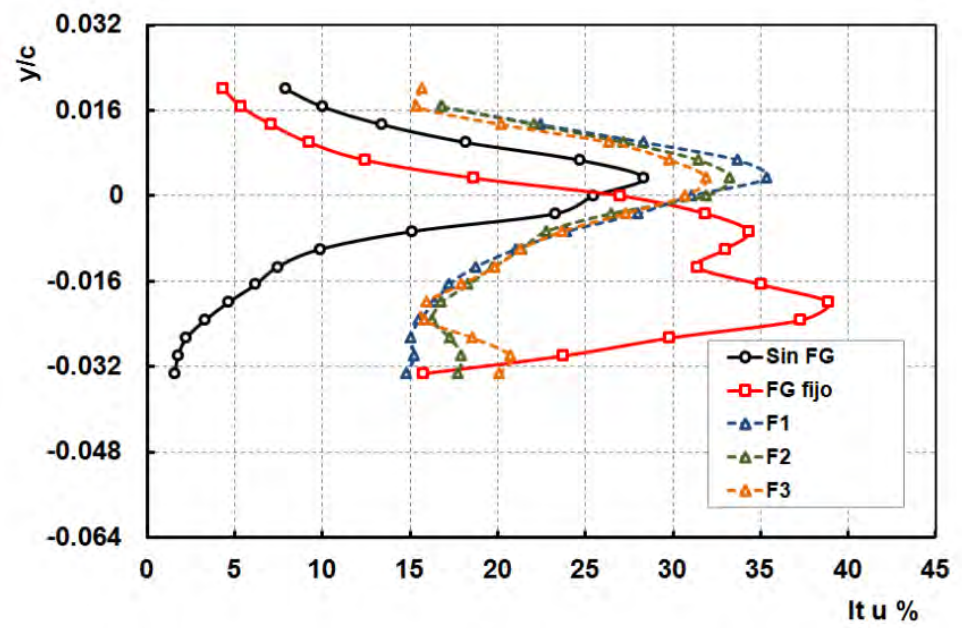

Figura 5.23: Perfil de intensidad de turbulencia "u" al 4\%c - Mini-flap Móvil.

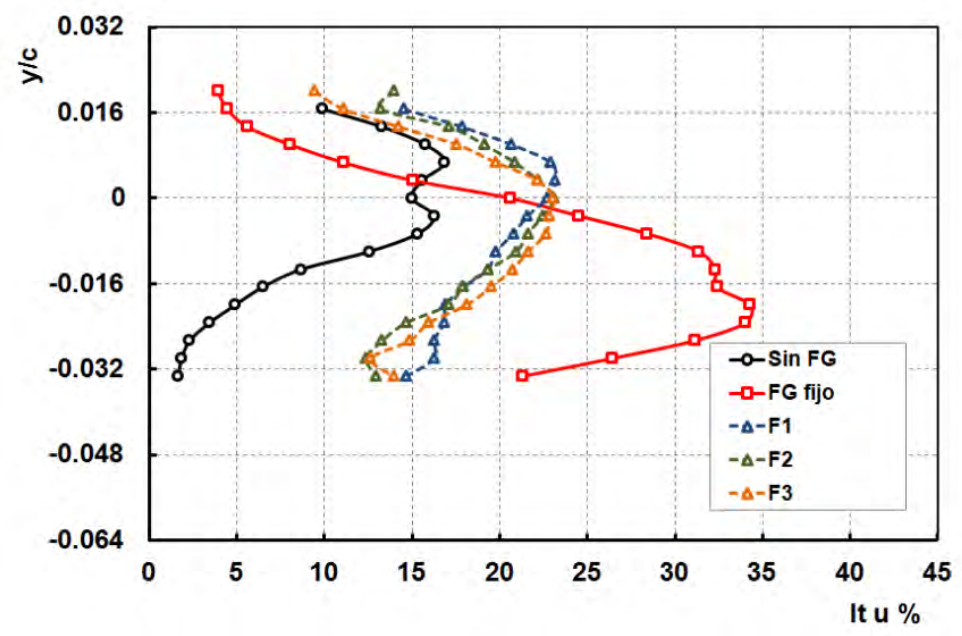

Figura 5.24: Perfil de intensidad de turbulencia "u" al 10\%c - Mini-flap Móvil.

\subsubsection{Comparación de Dispositivos - Intensidad de Turbulencia $u$}

Analizando la intensidad de turbulencia vemos que para el caso de perfil sin miniflap Gurney los niveles son menores respecto de los otros casos. También se ve que los picos son más pronunciados debido a la ausencia del mini-flap y se ubican a la altura del borde de fuga. Si analizamos solamente el caso de perfil con mini-flap Gurney fijo vemos que al 2\%c los valores son similares (alrededor del 30\%) y las curvas son más dispersas. Dichas curvas de intensidad de turbulencia decaen a medida que me alejo aguas abajo en los casos con dispositivos rotantes, no siendo así en el caso del dispositivo móvil manteniéndose aproximadamente constante al 30\%. Si bien los niveles de intensidad de turbulencia decaen aguas abajo, cabe mencionar que para el caso del mini-flap Gurney móvil dichos niveles decaen más lentamente respecto del ala con el dispositivo rotante de $30^{\circ}$. 
En los tres dispositivos se ve que al $2 \%$ c el pico de turbulencia se da para el caso de frecuencia 1. Esta tendencia se da en los casos con dispositivo rotante pero no para el móvil. En las crestas de los picos de las curvas se ve un mínimo presente en la mayoría de los casos el cual se acentúa aguas abajo.

Hay una tendencia de los valores de intensidad de turbulencia respecto de la frecuencia de activación. A mayor frecuencia entonces la intensidad disminuye. Dichas curvas se encuentran en medio de los casos sin y con mini-flap Gurney fijo tanto en sus valores como en su dispersión [26, 28, 33].

\subsubsection{Componente $V$}

\subsubsection{Caso Mini-flap Gurney Rotante}

Analizando las curvas (Figura 5.25) se observa que la menor intensidad se da para el caso de perfil sin flap Gurney, no siendo así para el resto de los casos, donde los valores picos son similares. Aquí los picos se dan un poco por debajo del borde de fuga y son de similar intensidad en los casos de frecuencia y Gurney fijo pero menos pronunciado en el caso con frecuencia.

Aguas abajo (Figura 5.26) los valores de intensidad de turbulencia ya son de menor intensidad para todos los casos respecto de $2 \%$ c. Existe una tendencia a medida que se modifica la frecuencia de activación. Si se incrementa la frecuencia entonces la intensidad de turbulencia se reduce. También se observa que los picos se dan a diferentes alturas.

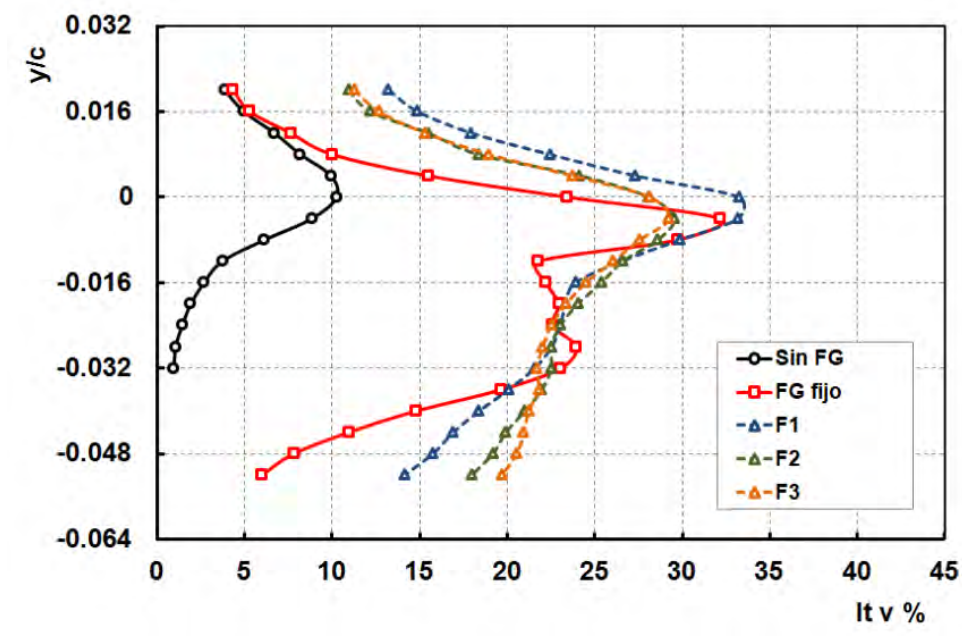

Figura 5.25: Perfil de intensidad de turbulencia "v" al 2\%c - Mini-flap Rotante. 


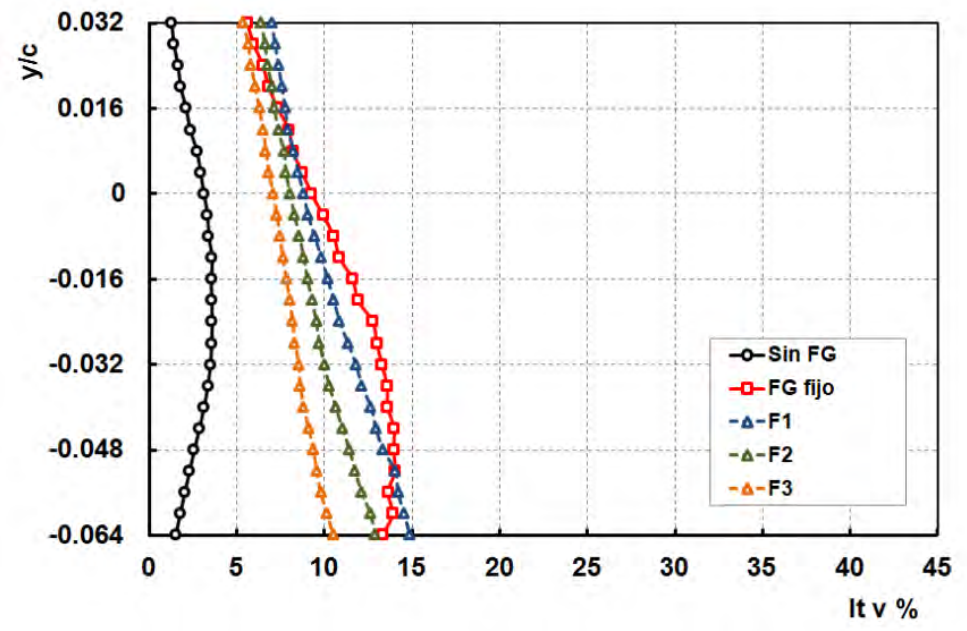

Figura 5.26: Perfil de intensidad de turbulencia "v" al 75\%c - Mini-flap Rotante.

\subsubsection{Caso Mini-flap Gurney Rotante (Variante $30^{\circ}$ )}

En este dispositivo, la intensidad de turbulencia también es menor en el caso del perfil sin mini-flap Gurney y los picos se dan por debajo del borde de fuga. Dichos picos son de similar intensidad para el caso de frecuencia y mini-flap Gurney fijo (Figura 5.27). Si nos movemos aguas abajo la intensidad disminuye como era de esperar (Figuras 5.28 y 5.29). El pico de mayor intensidad se da para el caso del perfil con mini-flap Gurney fijo. Para los casos con activación la mayor intensidad se da para la frecuencia 1 a $2 \%$ c y 4\%c.

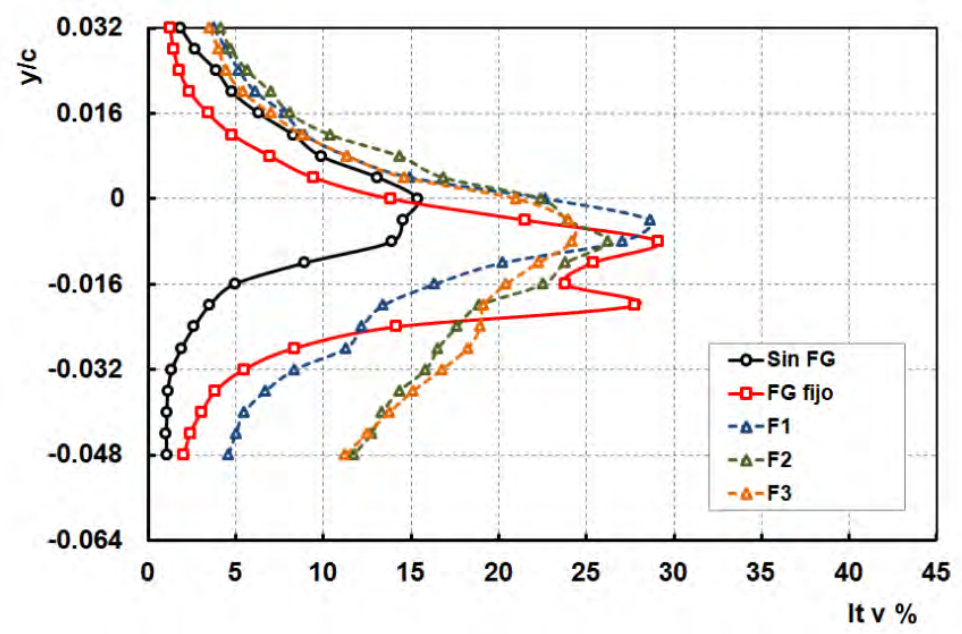

Figura 5.27: Perfil de intensidad de turbulencia "v" al 2\%c - Mini-flap Rotante $\left(30^{\circ}\right)$. 


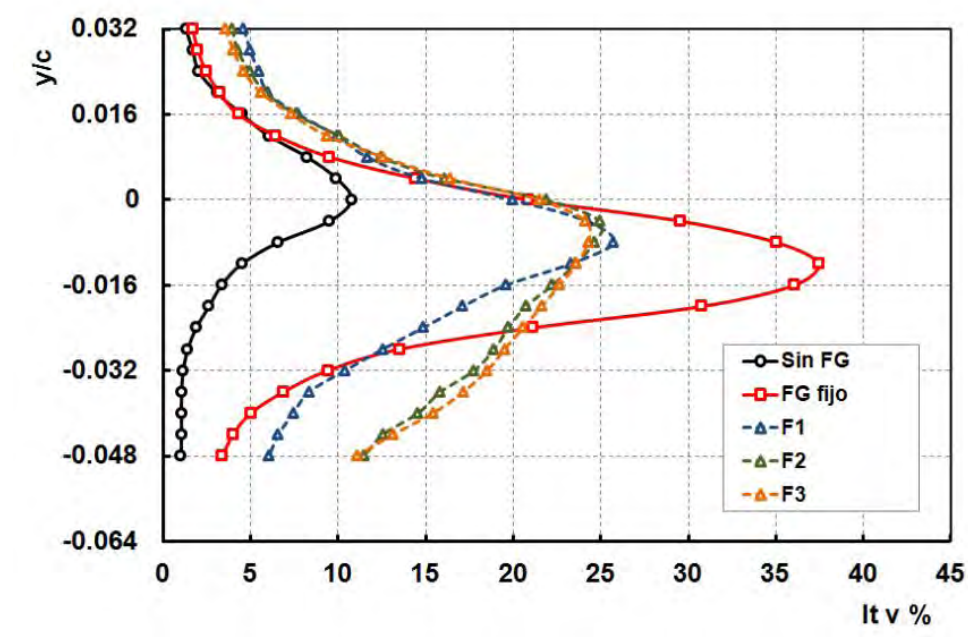

Figura 5.28: Perfil de intensidad de turbulencia "v" al 4\%c - Mini-flap Rotante $\left(30^{\circ}\right)$.

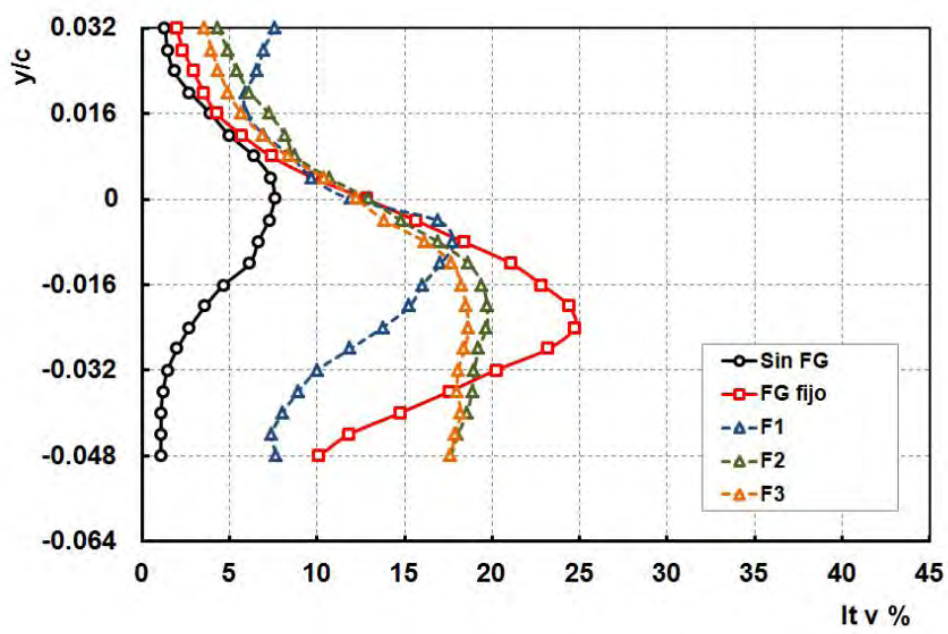

Figura 5.29: Perfil de intensidad de turbulencia "v" al 10\%c - Mini-flap Rotante $\left(30^{\circ}\right)$.

\subsubsection{Caso Mini-flap Gurney Móvil}

Nuevamente se observa que la menor intensidad de turbulencia se da para el caso del perfil sin mini-flap Gurney. Para los casos con frecuencia, el valor mayor se da para la frecuencia 1. En este dispositivo los picos se dan a la altura del borde de fuga a $2 \% \mathrm{c}$ (Figura 5.30) y 4\%c (Figura 5.31) para los casos de perfil solo y con frecuencia, no siendo así el caso del mini-flap Gurney fijo. La intensidad de turbulencia disminuye y se suaviza a medida que me alejo en la estela menos en el caso del mini-flap Gurney fijo que se mantiene relativamente constante (Figura 5.32). 


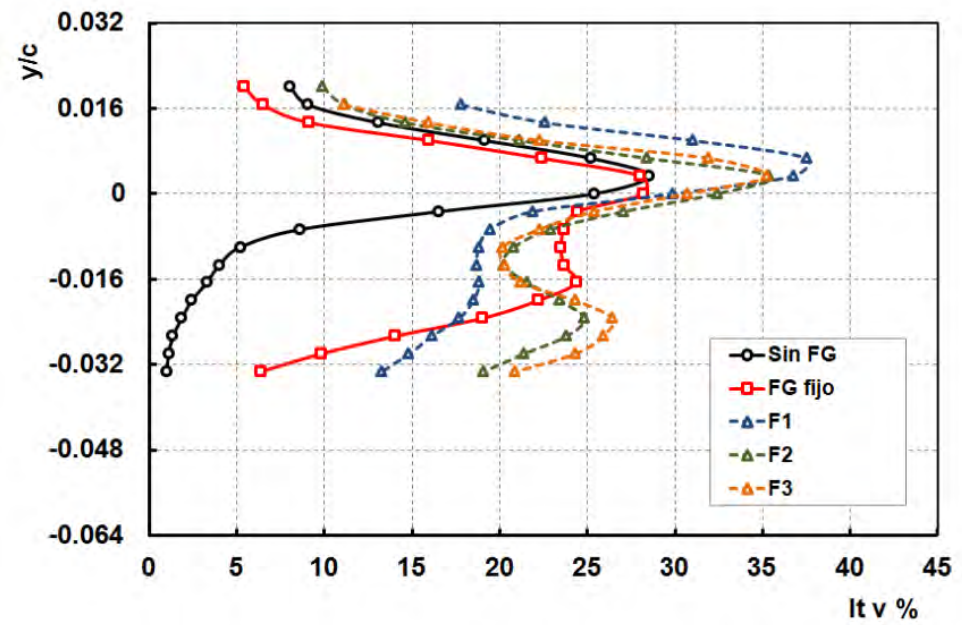

Figura 5.30: Perfil de intensidad de turbulencia "v" al 2\%c - Mini-flap Móvil.

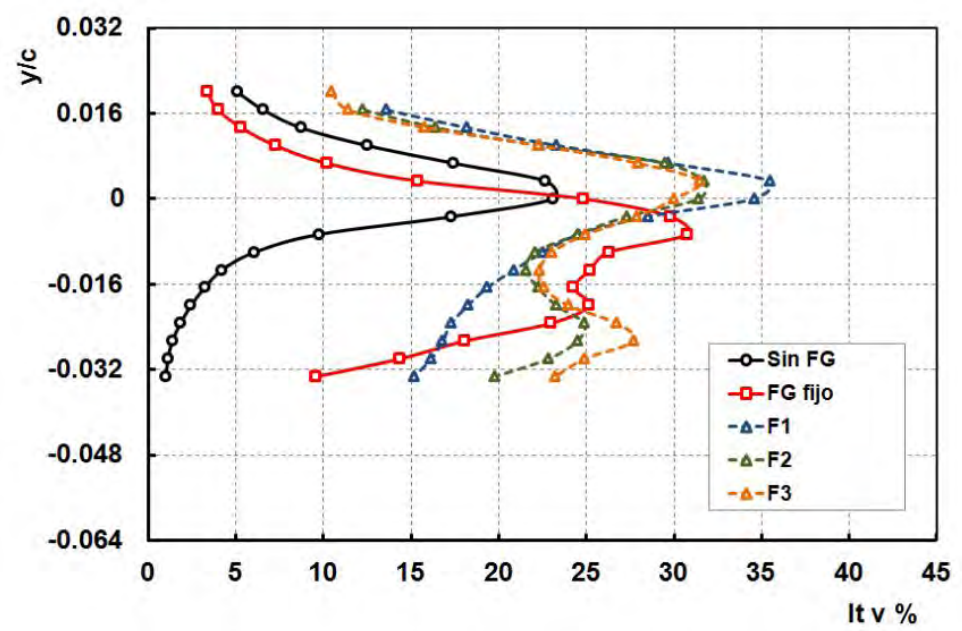

Figura 5.31: Perfil de intensidad de turbulencia "v" al 4\%c - Mini-flap Móvil.

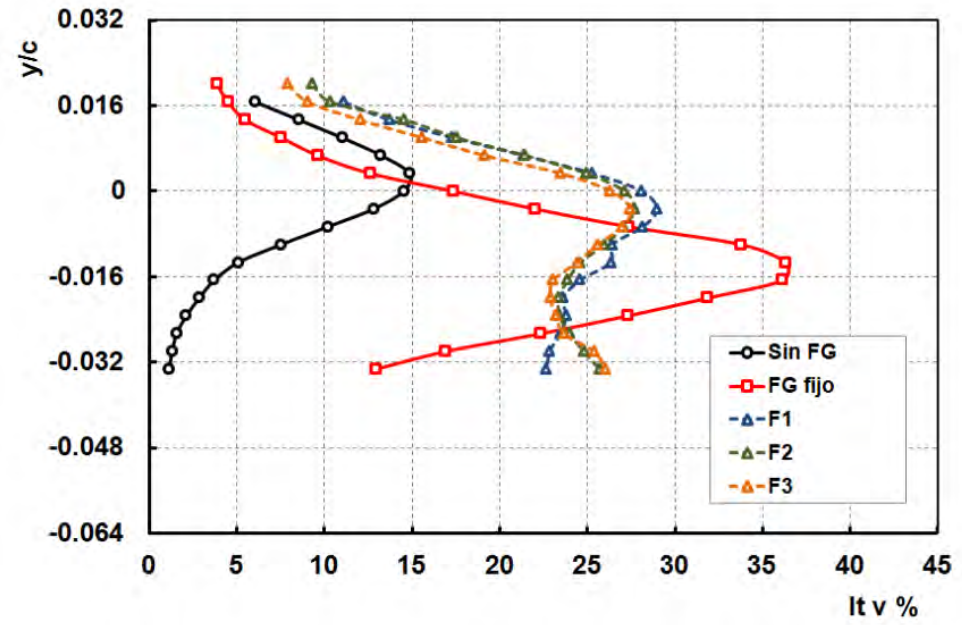

Figura 5.32: Perfil de intensidad de turbulencia "v" al 10\%c - Mini-flap Móvil. 


\subsubsection{Comparación de Dispositivos - Intensidad de Turbulencia $V$}

Analizando las curvas de la intensidad de turbulencia de la componente "v" vemos que los valores picos se dan entre el 30 y $35 \%$ al $2 \%$ c detrás del borde de fuga. Si nos movemos aguas abajo los niveles decrecen por debajo del $20 \%$ en los casos rotatorios no siendo así en el caso móvil que lo hace más lentamente (25 a 30\%).

Se ve también que en la estela cercana $(2 \%$ c) predominan los niveles de turbulencia de dispositivos activos pero pierden intensidad aguas abajo. Al igual que en la componente horizontal se ve que la mayor intensidad se alcanza para la frecuencia 1 y a medida que esta aumenta la intensidad disminuye. 


\subsection{Vectores de Velocidad Media}

En esta sección se analizan los vectores de velocidad media en la estela del perfil para los diferentes dispositivos y diferentes frecuencias de activación. Se tuvieron en cuenta solo ciertos puntos de interés en el "eje y" para no entorpecer el análisis ( $0 \%$ c y $1.6 \%$ c). En el caso mini-flap Gurney Rotante se analizan los puntos al 2\%c y $75 \%$ c, mientras que en el caso mini-flap Gurney Rotante (Variante $30^{\circ}$ ) y el caso mini-flap Gurney móvil se analizan los puntos al 2\%c, 4\%c y 10\%c detrás del borde de fuga por lo que las escalas en el "eje $\mathrm{x}$ " $\mathrm{y}$ "eje y" tienen diferentes rangos. También hay que mencionar que el análisis de dichas gráficas no pretende comparar las magnitudes de dichos vectores sino estudiar el campo de vectores de velocidades medias a fin de poder determinar la función de cada dispositivo. Se optó por magnificar la escala de la componente $v$ con un factor "dos" a modo de poder visualizar las diferencias en esta componente.

\subsubsection{Caso Mini-flap Gurney Rotante}

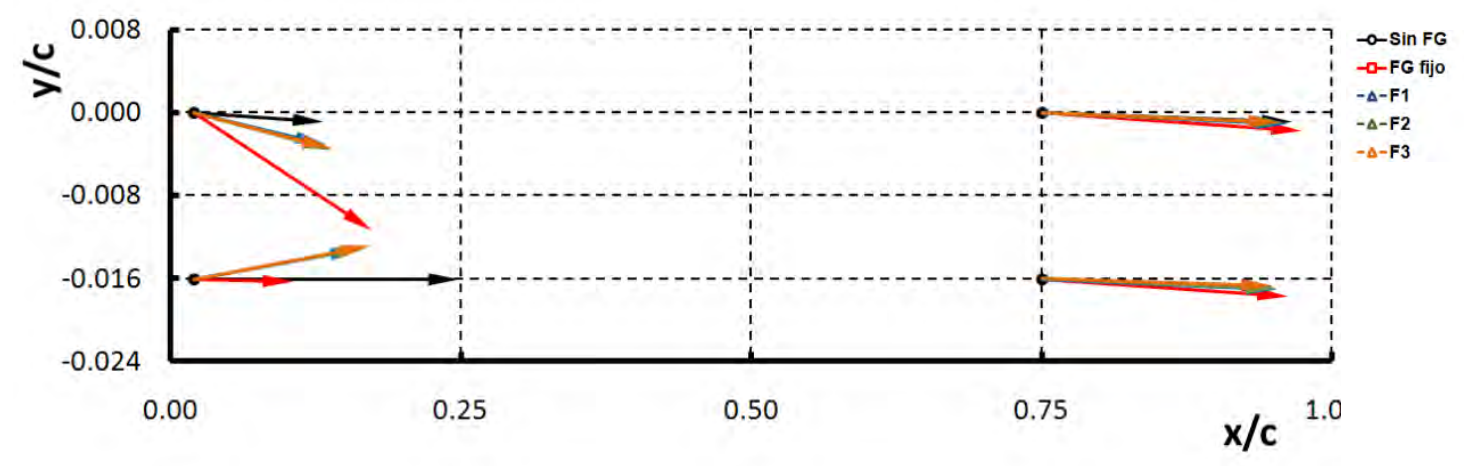

Figura 5.33: Diagrama de vectores de velocidad media - Mini-flap Rotante.

Analizando el caso mini-flap Gurney Rotante (Figura 5.33) se observa la influencia del mini-flap fijo sobre la componente vertical de la velocidad en la estela cercana. Los casos con frecuencia también producen variaciones en dicha componente pero con menor intensidad. No se aprecia una gran diferencia si analizamos las diferentes frecuencias de activación, ya que casualmente en dichos puntos no se dan variaciones (dichas diferencias se aprecian en los perfiles de velocidad media descriptos anteriormente). Si miramos los vectores al $75 \%$ c vemos que la influencia de los diferentes dispositivos ya no es tan pronunciada lo cual nos da una idea de que la mayor influencia de dichos dispositivos se da en la estela cercana lo que se corresponde con estudios de diferentes autores que concluyen que la estela se vuelve simétrica a una cuerdas por detrás del borde de fuga y que es la asimetría de los vórtices producidos por dichos mecanismos en la estela cercana lo que produce variaciones en la circulación del perfil y por ende en el "downwash". 


\subsubsection{Caso Mini-flap Gurney Rotante (Variante $30^{\circ}$ )}

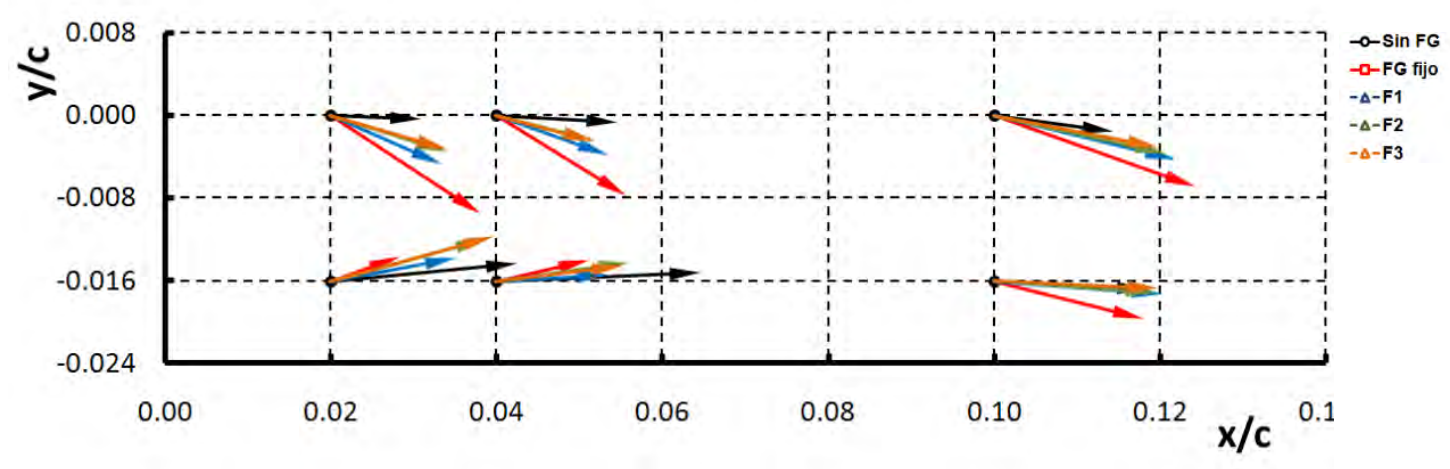

Figura 5.34: Diagrama de vectores de velocidad media - Mini-flap Rotante $\left(30^{\circ}\right)$.

Analizando el caso mini-flap Rotante (Variante $30^{\circ}$ ) (Figura 5.34) vemos que nuevamente el perfil con mini-flap fijo es el mecanismo que introduce mayores modificaciones en las componentes verticales de velocidad media en las tres posiciones detrás del borde de fuga. Si lo comparamos con el perfil sin dispositivos existe una gran diferencia por lo que podríamos deducir que hay un aumento de la corriente descendente con un consecuente aumento de sustentación.

Si tenemos en cuenta solamente las diferentes frecuencias, vemos que la frecuencia que produce mayor perturbación en la componente vertical es la frecuencia 1. Existe una tendencia respecto de la frecuencia de activación. Si aumentamos dicho valor entonces disminuye la componente vertical de velocidad media. Esta tendencia se repite a diferentes posiciones.

\subsubsection{Caso Mini-flap Gurney Móvil}

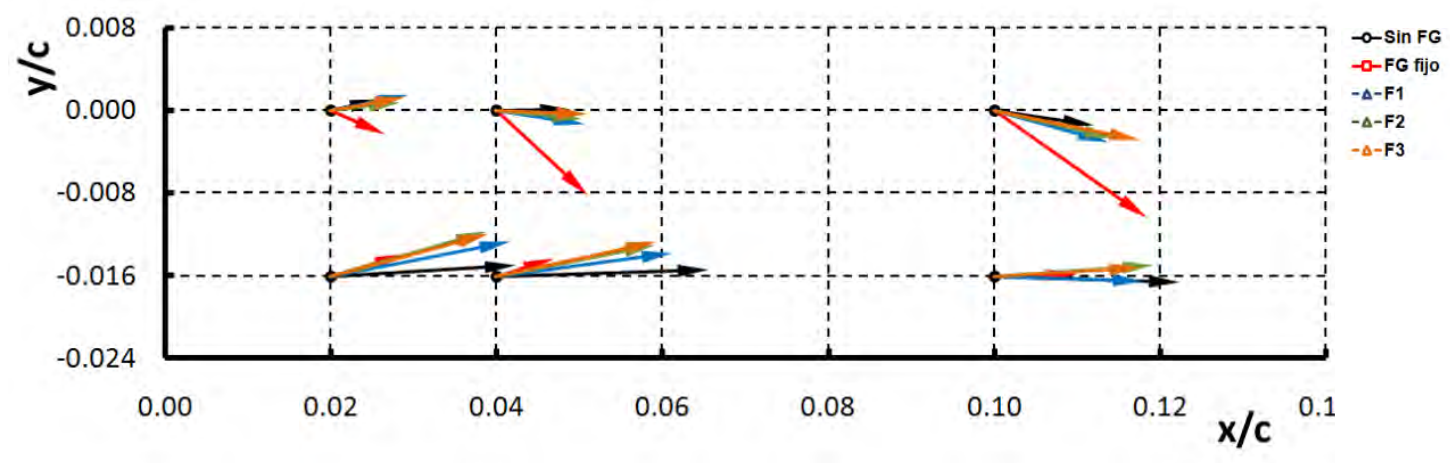

Figura 5.35: Diagrama de vectores de velocidad media - Mini-flap Móvil. 
Para el caso mini-flap Móvil (Figura 5.35) vemos que en este caso también es el mini-flap fijo el dispositivo que introduce mayores cambios en la estela del perfil. Los casos con frecuencia al igual que en los casos anteriores se encuentran entre los casos de mini-flap fijo y sin dispositivos. Existe una tendencia donde para las frecuencias menores se da el mayor "downwash" tanto en la zona superior como en la inferior. En la zona inferior se ve que los vectores correspondientes al mini-flap fijo son de menor magnitud que el resto. Este comportamiento se da en los diferentes mecanismos y se invierte en el caso del perfil sin mecanismos. En cambio los vectores a la altura del borde de fuga se da que la magnitud es mayor para el caso del mini-flap fijo y la menor para el perfil solo. El análisis de ambos vectores en conjunto nos podría dar una idea de cómo fluctúa la circulación y por ende la sustentación.

\subsection{Escalas Espaciales de Turbulencia}

De las curvas de coeficiente de auto-correlación se obtienen las escalas temporales mediante el criterio de "cruce por cero". Con este valor y con la velocidad media en el punto de análisis se obtienen las escalas espaciales tanto para la componente $u$ como la componente $v$ de acuerdo a la hipótesis de flujo congelado. En las gráficas se observan elipses donde la dimensión de los ejes representa las escalas espaciales (escalas de las fluctuaciones de la componente $u$ en abscisas y de la componente $v$ en ordenadas). De este modo es posible comparar los diferentes casos de estudio. Asimismo, los límites de los ejes difieren ya que las escalas de la componente $v$ son mucho menores que las de la componente $u$, por lo que era conveniente disminuir los límites de las escalas de $v$.

Si se observan las magnitudes de ambas escalas es fácil deducir que las escalas correspondientes a la componente longitudinal $u$ son aproximadamente diez veces más grandes que las escalas de la componente $v$.

Analizando las gráficas (Figura 5.36 a 5.51) se observa que las escalas del perfil sin mini-flap Gurney tienden a ser más chicas ya que se encuentran a la altura del borde de fuga con lo que podríamos decir que no hay influencia de las escalas más grandes pertenecientes a las escalas del perfil en general sino de las escalas del borde de fuga, mientras que a la altura del borde de fuga del mini-flap Gurney son más grandes en $u$ con influencia de las escalas de las capas exteriores.

Las escalas del mini-flap Gurney fijo están en el orden de magnitud "2" en la componente $u$ y menores a "0.4" en la componente $v$. Esto nos da una idea de que diferentes dispositivos producen estructuras con escalas similares en la estela del perfil. Asimismo dichas escalas de la componente $v$ tienden a disminuir a medida que me alejo aguas abajo mientras que la componente $u$ se comporta de forma inversa, es decir aumentan su escala. Este fenómeno se observa en los tres modelos y para diferentes casos (con y sin actuación). 
Si se analizan solamente los casos con frecuencia de actuación se ve que en la mayoría de los casos las curvas son concéntricas existiendo una relación entre la frecuencia y las escalas espaciales tanto en la componente $u$ como en $v$. De esta forma, podemos deducir que si aumento la frecuencia de activación entonces las escalas se reducen.

Si comparamos los diferentes dispositivos vemos que el que produce escalas más grandes tanto en $u$ como en $v$ es el mecanismo de mini-flap Gurney rotante (variante $30^{\circ}$ ) para el caso de la frecuencia 1. Esto se puede explicar si pensamos que el tipo de mecanismo utilizado es de rotación introduciendo movimiento al fluido circundante, mientras que el mecanismo del mini-flap Gurney móvil genera estructuras con escalas importantes solo en $u$, donde el mini-flap en este caso asciende y desciende en forma alternada sin introducir un movimiento en el fluido en la dirección de $v$.

En algunos casos (Figuras 5.36, 5.46, 5.48 y 5.50) se observa que las escalas espaciales de la componente $v$ para el caso del Gurney fijo son mayores que las escalas producidas por los dispositivos activos. Si pensamos que en la determinación de dichas escalas la velocidad media en el punto es una de las variables que tiene influencia y que en la mayoría de los casos es el mini-flap Gurney fijo el que produce mayor "downwash", entonces las escalas tenderán a incrementar su tamaño. Podemos decir de alguna forma que si las escalas en la componente $v$ aumentan su tamaño, entonces la sustentación se vería afectada $[25,33]$.

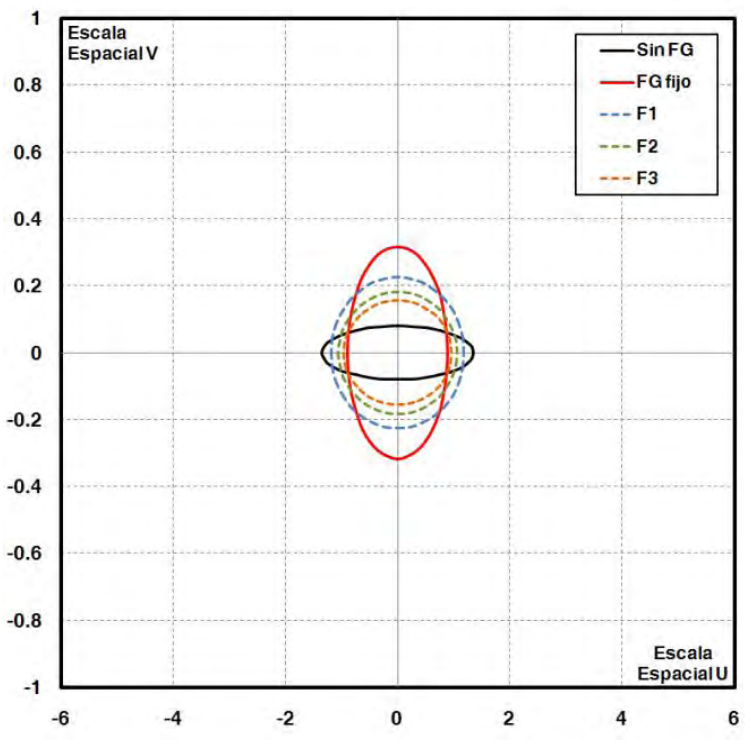

Figura 5.36: Escalas espaciales Mini-flap Rotante $(\mathrm{x} / \mathrm{c}=2 \% \mathrm{c}, \mathrm{y} / \mathrm{c}=0 \% \mathrm{c})$. 


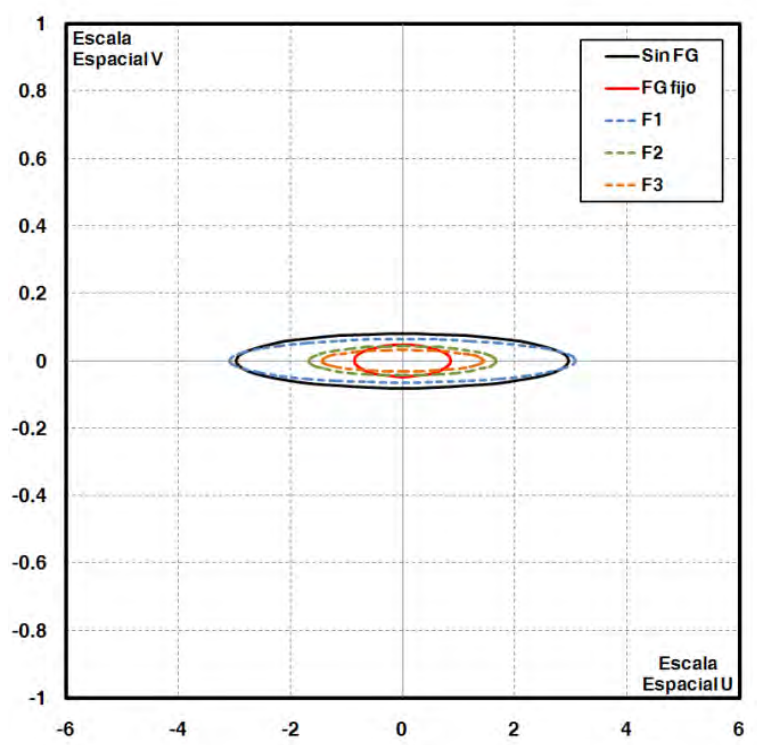

Figura 5.37: Escalas espaciales Mini-flap Rotante $(\mathrm{x} / \mathrm{c}=2 \% \mathrm{c}, \mathrm{y} / \mathrm{c}=-2 \% \mathrm{c})$.

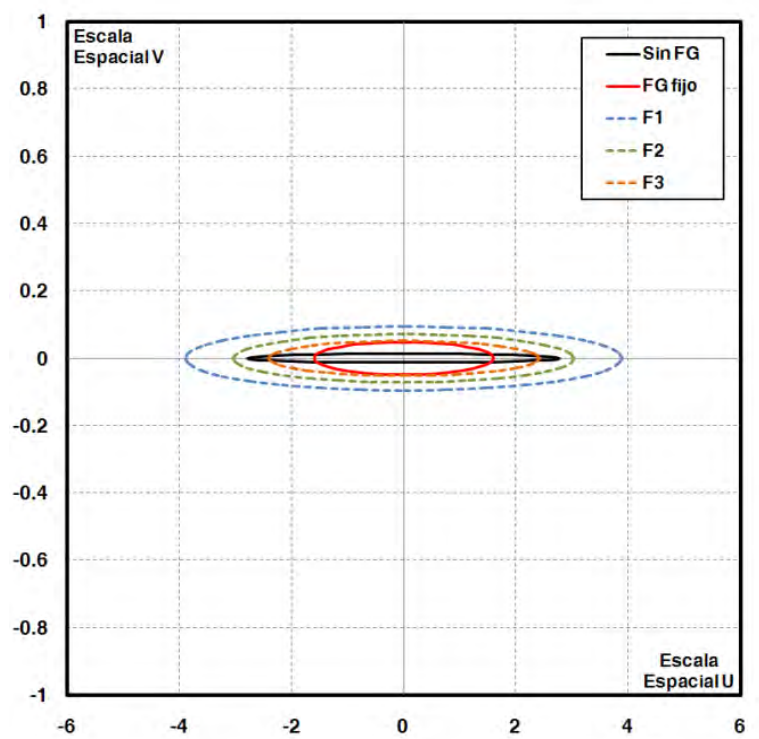

Figura 5.38: Escalas espaciales Mini-flap Rotante (x/c=75\%c, y/c=0\%c). 


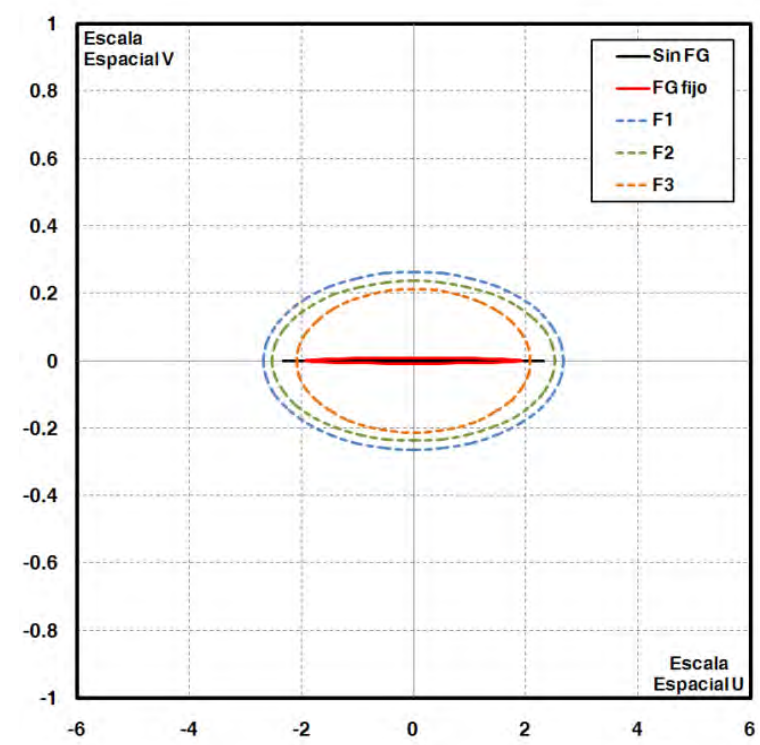

Figura 5.39: Escalas espaciales Mini-flap Rotante $(\mathrm{x} / \mathrm{c}=75 \% \mathrm{c}, \mathrm{y} / \mathrm{c}=-2 \% \mathrm{c})$.

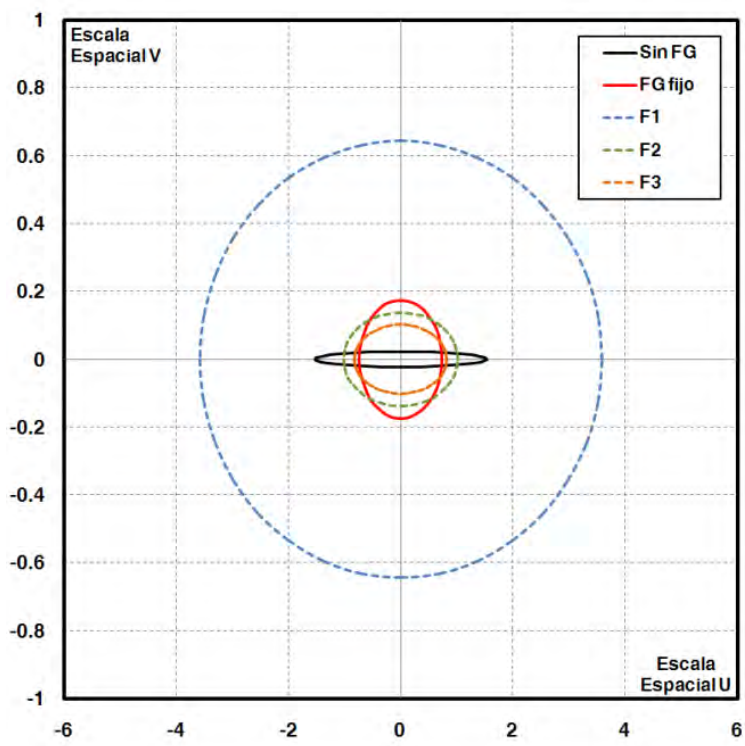

Figura 5.40: Escalas espaciales Mini-flap Rotante $\left(30^{\circ}\right)(\mathrm{x} / \mathrm{c}=2 \% \mathrm{c}, \mathrm{y} / \mathrm{c}=0 \% \mathrm{c})$. 


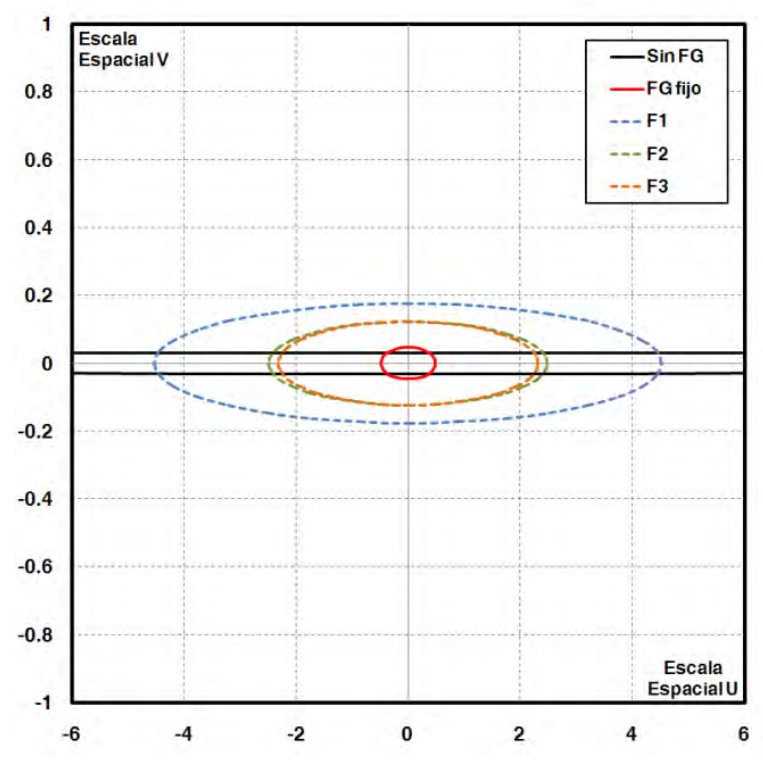

Figura 5.41: Escalas espaciales Mini-flap Rotante $\left(30^{\circ}\right)(\mathrm{x} / \mathrm{c}=2 \% \mathrm{c}, \mathrm{y} / \mathrm{c}=-2 \% \mathrm{c})$.

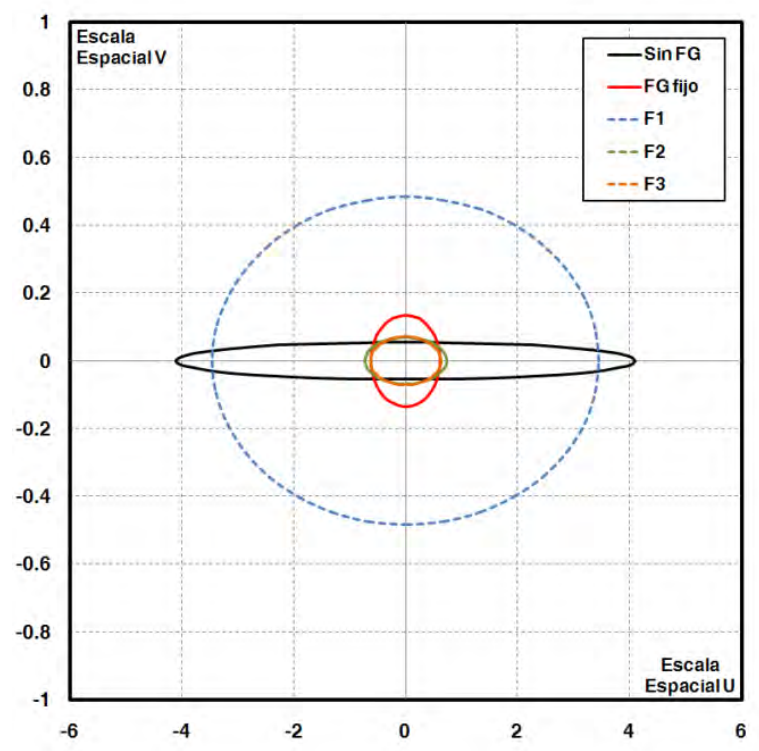

Figura 5.42: Escalas espaciales Mini-flap Rotante $\left(30^{\circ}\right)(\mathrm{x} / \mathrm{c}=4 \% \mathrm{c}, \mathrm{y} / \mathrm{c}=0 \% \mathrm{c})$. 


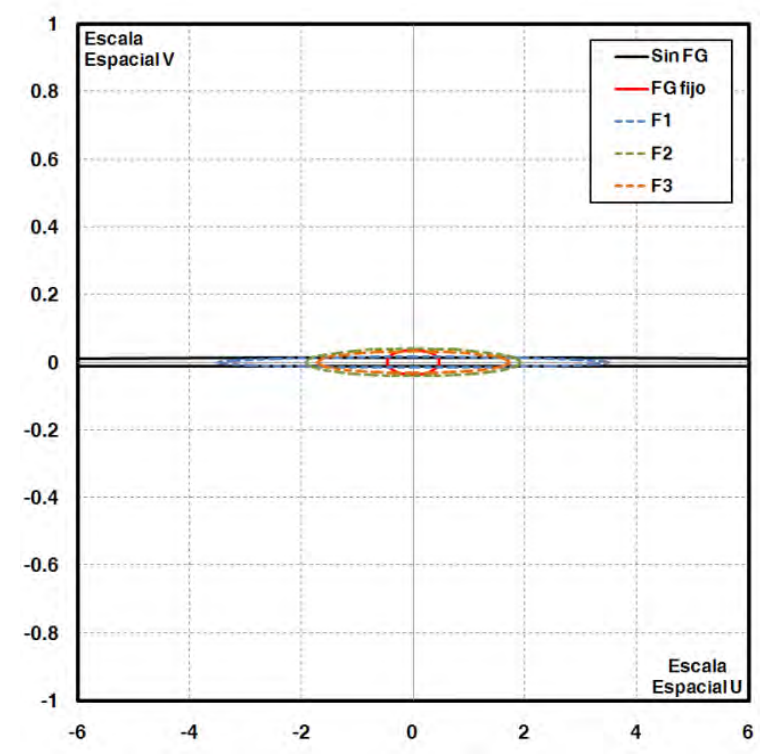

Figura 5.43: Escalas espaciales Mini-flap Rotante $\left(30^{\circ}\right)(\mathrm{x} / \mathrm{c}=4 \% \mathrm{c}, \mathrm{y} / \mathrm{c}=-2 \% \mathrm{c})$.

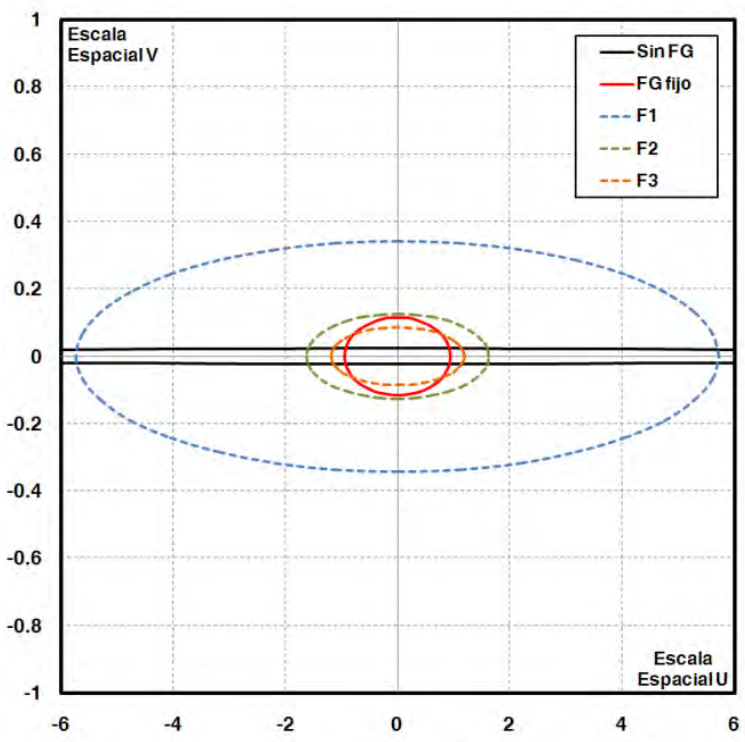

Figura 5.44: Escalas espaciales Mini-flap Rotante $\left(30^{\circ}\right)(\mathrm{x} / \mathrm{c}=10 \% \mathrm{c}, \mathrm{y} / \mathrm{c}=0 \% \mathrm{c})$. 


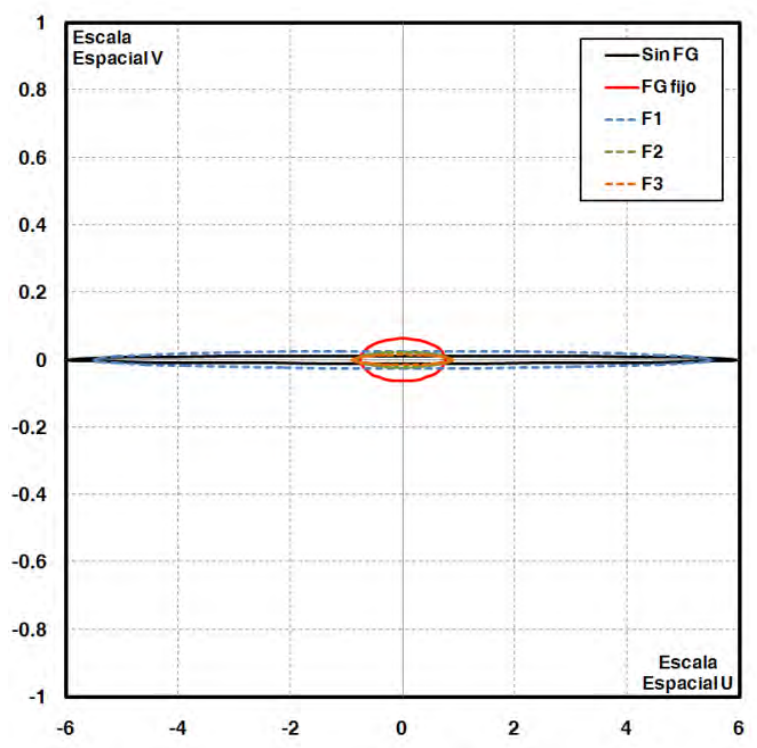

Figura 5.45: Escalas espaciales Mini-flap Rotante $\left(30^{\circ}\right)(\mathrm{x} / \mathrm{c}=10 \% \mathrm{c}, \mathrm{y} / \mathrm{c}=-2 \% \mathrm{c})$.

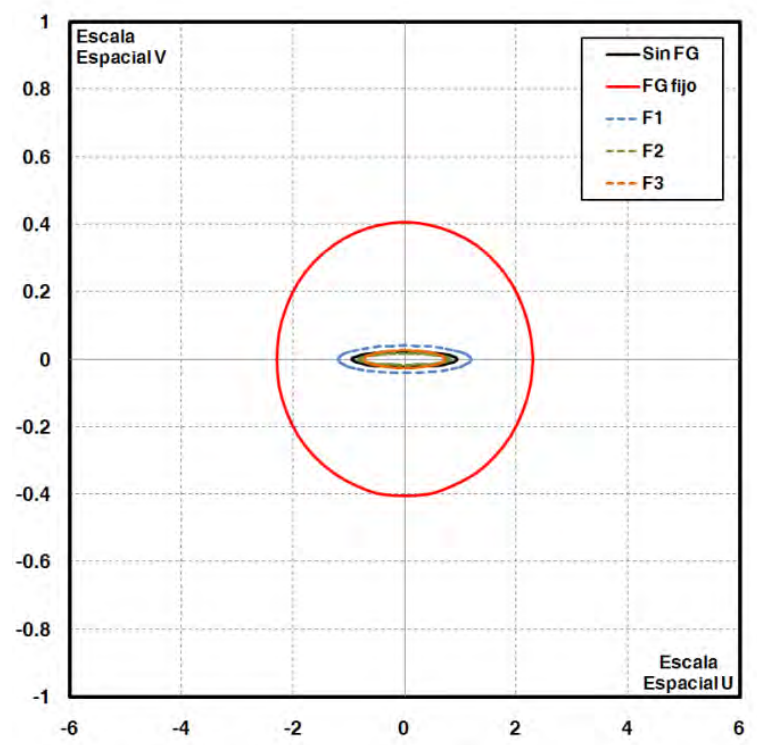

Figura 5.46: Escalas espaciales Mini-flap Móvil (x/c=2\%c, y/c=0\%c). 


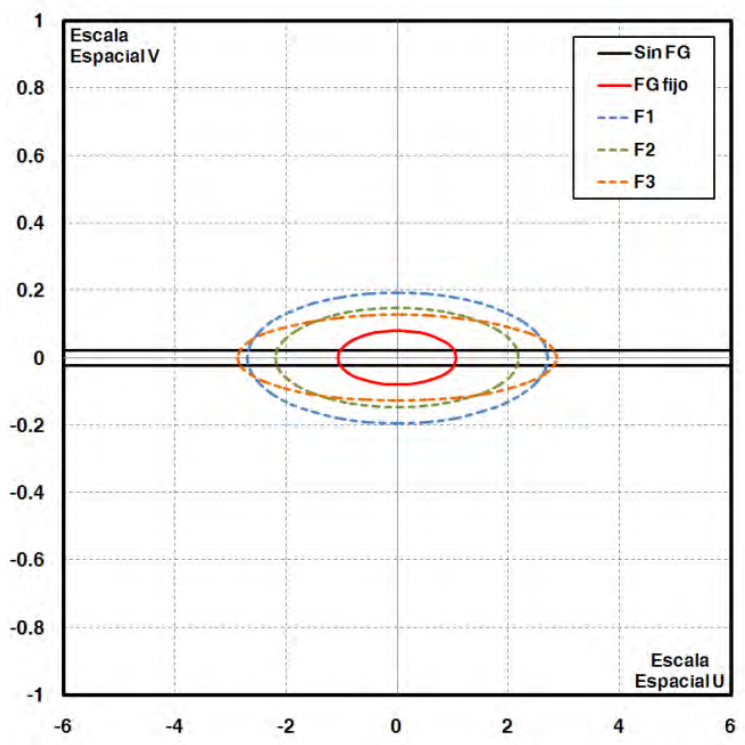

Figura 5.47: Escalas espaciales Mini-flap Móvil (x/c=2\%c, y/c=-2\%c).

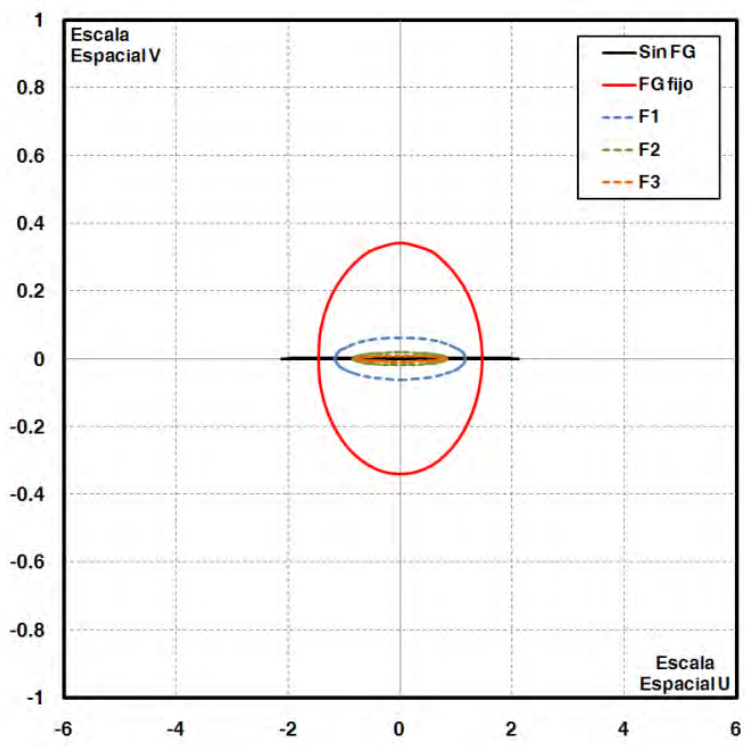

Figura 5.48: Escalas espaciales Mini-flap Móvil (x/c=4\%c, y/c=0\%c). 


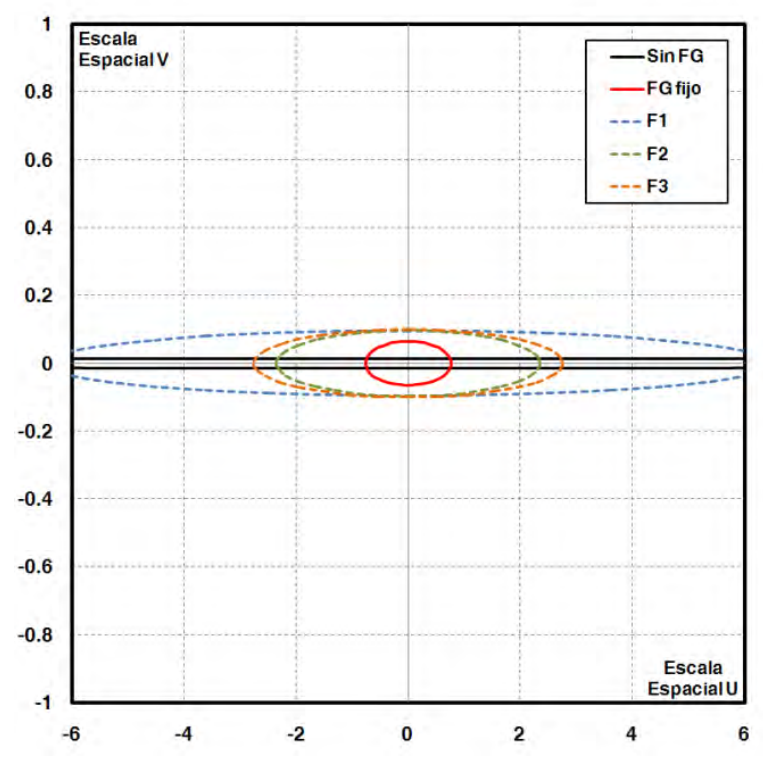

Figura 5.49: Escalas espaciales Mini-flap Móvil (x/c=4\%c, y/c=-2\%c).

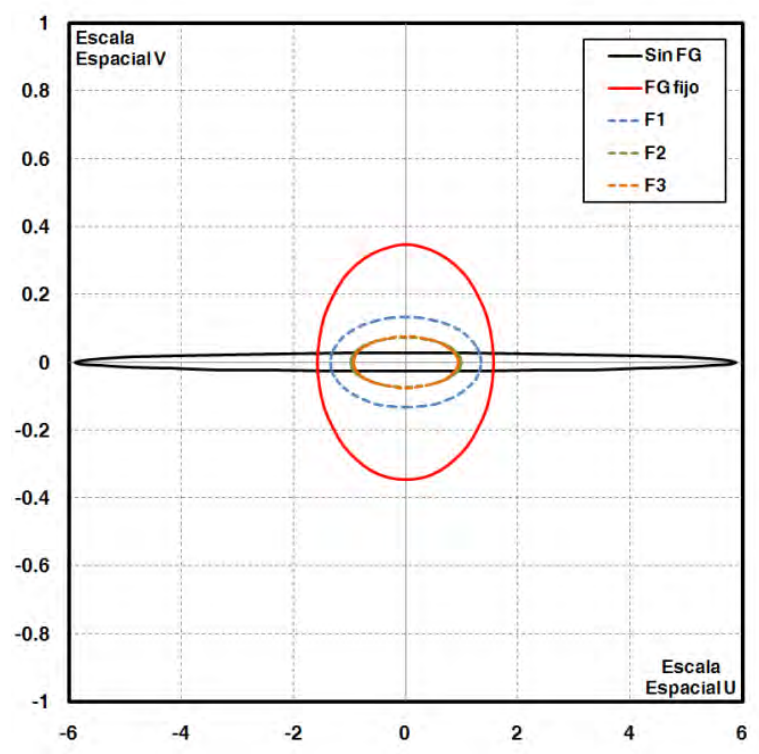

Figura 5.50: Escalas espaciales Mini-flap Móvil (x/c=10\%c, y/c=0\%c). 


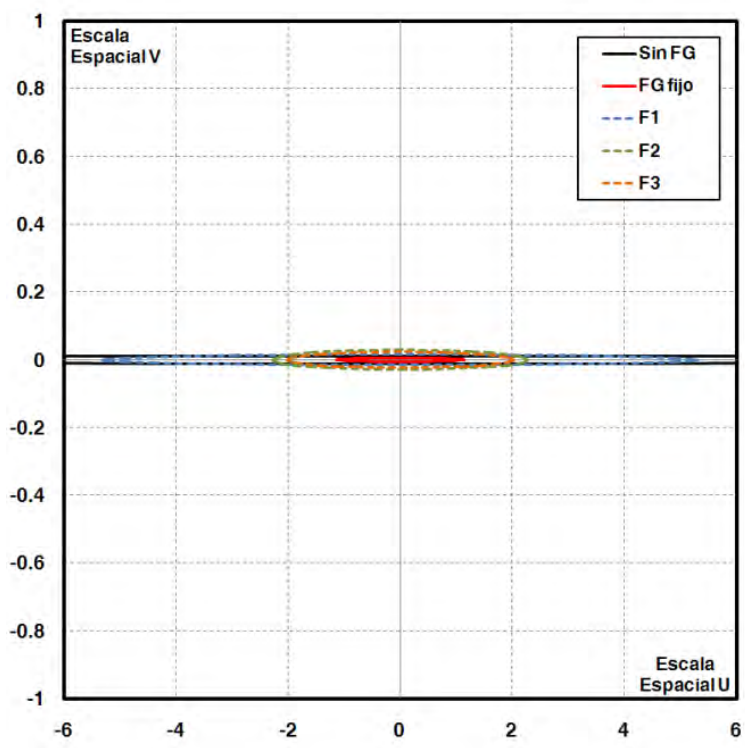

Figura 5.51: Escalas espaciales Mini-flap Móvil (x/c=10\%c, y/c=-2\%c).

\subsection{Espectros de Densidad de Potencia (PSD)}

Se analizan los espectros de potencia correspondientes a la componente "v" en los puntos de interés como ya se mencionó anteriormente. En dichos gráficos se observa la existencia o no de fenómenos periódicos como ser desprendimientos de estructuras fluidodinámicas detrás del mini-flap Gurney. Estos fenómenos se manifiestan en forma de picos predominantes de potencia a una determinada frecuencia. Con este análisis se pretende determinar la relación entre las frecuencias de desprendimiento detrás del perfil y las frecuencias de actuación como así también la energía de dichas estructuras respecto del perfil con mini-flap Gurney fijo. En la Figura 5.52 se muestra un gráfico típico para dicho análisis.

Los valores de los picos de potencia son adimencionalizados de la siguiente forma:

$$
P S D_{\text {adim. }}=\frac{P S D \cdot f}{U_{\infty}^{2}}
$$

donde $P S D$ es el valor del pico de potencia, $f$ es la frecuencia correspondiente al pico y $U_{\infty}$ es la velocidad de la corriente libre. 


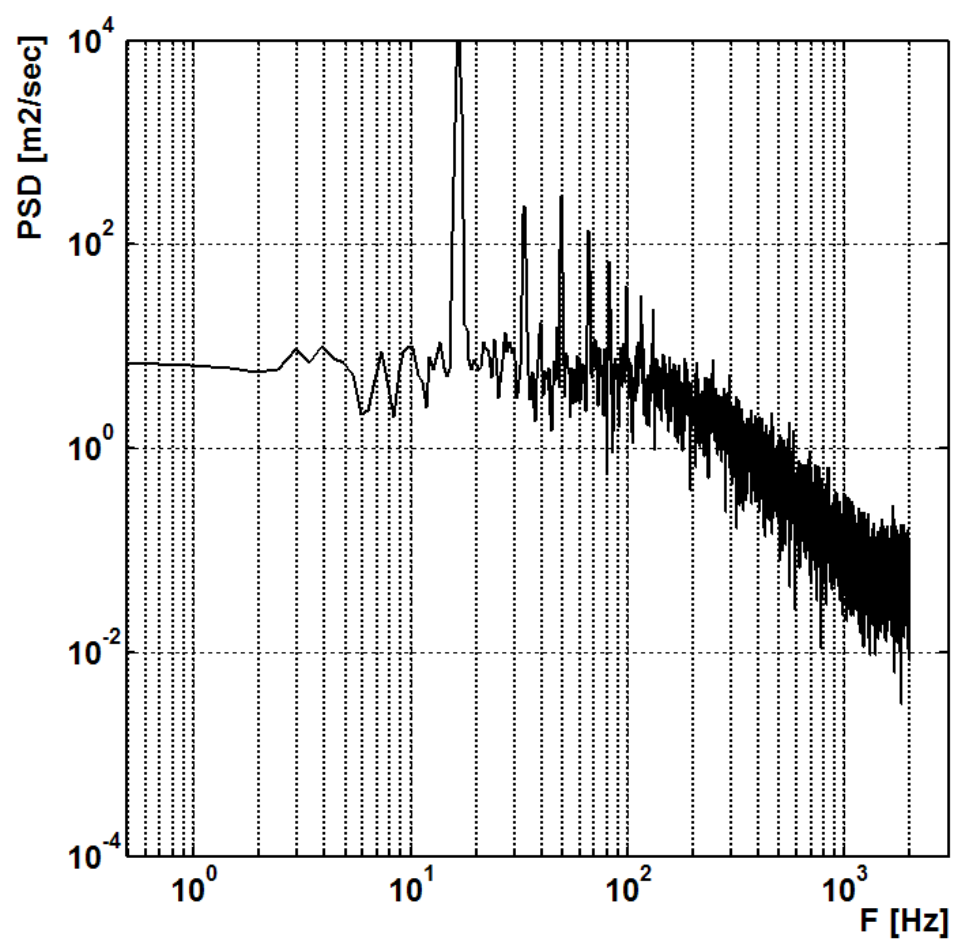

Figura 5.52: Espectro de densidad de potencia Mini-flap Rotante F1 $(\mathrm{x} / \mathrm{c}=10 \% \mathrm{c}, \mathrm{y} / \mathrm{c}=-2 \% \mathrm{c})$.

Por otro lado los valores de frecuencia se adimencionalizan utilizando el número de Strouhal como sigue:

$$
S t=\frac{f . h}{U_{\infty}}
$$

donde $h$ es la altura del flap Gurney. De la misma forma se calculan las frecuencias reducidas $k$ de activación de la siguiente forma:

$$
k=\frac{F \cdot h}{U_{\infty}}
$$

donde $F$ es la frecuencia de activación de los mecanismos. La siguiente tabla muestra las diferentes frecuencias reducidas para los diferentes mecanismos de acuerdo a la altura del mini-flap Gurney: 


\begin{tabular}{ccccc}
\hline & & $\begin{array}{c}\text { Rotante } \\
\mathrm{h}_{1}=0.01 \mathrm{~m}\end{array}$ & $\begin{array}{c}\text { Rotante }\left(30^{\circ}\right) \\
\mathrm{h}_{2}=0.01 \mathrm{~m}\end{array}$ & $\begin{array}{c}\text { Móvil } \\
\mathrm{h}_{3}=0.008 \mathrm{~m}\end{array}$ \\
\hline $\mathrm{F}_{1}=22 \mathrm{~Hz}$ & $\mathrm{k}_{1}$ & 0.022 & 0.022 & 0.018 \\
$\mathrm{~F}_{2}=38 \mathrm{~Hz}$ & $\mathrm{k}_{2}$ & 0.038 & 0.038 & 0.030 \\
$\mathrm{~F}_{3}=44 \mathrm{~Hz}$ & $\mathrm{k}_{3}$ & 0.044 & 0.044 & 0.035 \\
\hline
\end{tabular}

Tabla 5.1: Frecuencia reducida para diferentes mecanismos.

Con los valores adimensionalizados se confeccionan las siguientes tablas para los diferentes puntos de interés:

\begin{tabular}{ccccccc}
\hline & \multicolumn{2}{c}{$\mathrm{x} / \mathrm{c}=2 \% \mathrm{c}$} & \multicolumn{2}{c}{$\mathrm{x} / \mathrm{c}=75 \% \mathrm{c}$} & \multicolumn{2}{c}{-} \\
& Strouhal & $\mathrm{PSD}_{\text {adim. }}$ & Strouhal & PSD $_{\text {adim. }}$ & Strouhal & PSD $_{\text {adim. }}$ \\
\cline { 2 - 7 } FG fijo & 0.094 & 164 & 0.095 & 68 & - & - \\
$\mathrm{k}_{1}$ & 0.023 & 97 & 0.025 & 233 & - & - \\
$\mathrm{k}_{2}$ & 0.033 & 607 & 0.031 & 294 & - & - \\
$\mathrm{k}_{3}$ & 0.038 & 750 & 0.038 & 119 & - & - \\
\hline
\end{tabular}

Tabla 5.2: Strouhal y PSD adimensional vs. $k$ (Mini-flap Rotante, y/c $=0 \%$ c).

\begin{tabular}{ccccccc}
\hline & \multicolumn{2}{c}{$\mathrm{x} / \mathrm{c}=2 \% \mathrm{c}$} & \multicolumn{2}{c}{$\mathrm{x} / \mathrm{c}=4 \% \mathrm{c}$} & \multicolumn{2}{c}{$\mathrm{x} / \mathrm{c}=10 \% \mathrm{c}$} \\
& Strouhal & $\mathrm{PSD}_{\text {adim. }}$ & Strouhal & $\mathrm{PSD}_{\text {adim. }}$ & Strouhal & PSD $_{\text {adim. }}$ \\
\cline { 2 - 7 } FG fijo & 0.142 & 267 & 0.140 & 192 & 0.116 & 297 \\
$\mathrm{k}_{1}$ & 0.016 & 178 & 0.019 & 1333 & 0.014 & 163 \\
$\mathrm{k}_{2}$ & 0.035 & 287 & 0.038 & 81 & 0.029 & 213 \\
$\mathrm{k}_{3}$ & 0.041 & 242 & 0.040 & 80 & 0.034 & 76 \\
\hline
\end{tabular}

Tabla 5.3: Strouhal y PSD adimensional vs. $k$ (Mini-flap Rotante $30^{\circ}, \mathrm{y} / \mathrm{c}=0 \% \mathrm{c}$ ).

\begin{tabular}{ccccccc}
\hline & \multicolumn{2}{c}{$\mathrm{x} / \mathrm{c}=2 \% \mathrm{c}$} & \multicolumn{2}{c}{$\mathrm{x} / \mathrm{c}=4 \% \mathrm{c}$} & \multicolumn{2}{c}{$\mathrm{x} / \mathrm{c}=10 \% \mathrm{c}$} \\
& Strouhal & $\mathrm{PSD}_{\text {adim. }}$ & Strouhal & $\mathrm{PSD}_{\text {adim. }}$ & Strouhal & PSD $_{\text {adim. }}$ \\
\cline { 2 - 7 } FG fijo & 0.080 & 14 & 0.069 & 52 & 0.082 & 148 \\
$\mathrm{k}_{1}$ & 0.018 & 132 & 0.017 & 95 & 0.017 & 162 \\
$\mathrm{k}_{2}$ & 0.031 & 311 & 0.032 & 223 & 0.033 & 284 \\
$\mathrm{k}_{3}$ & 0.035 & 160 & 0.034 & 257 & 0.035 & 347 \\
\hline
\end{tabular}

Tabla 5.4: Strouhal y PSD adimensional vs. $k$ (Mini-flap Móvil, y/c $=0 \%$ c). 


\begin{tabular}{ccccccc}
\hline & \multicolumn{2}{c}{$\mathrm{x} / \mathrm{c}=2 \% \mathrm{c}$} & \multicolumn{2}{c}{$\mathrm{x} / \mathrm{c}=75 \% \mathrm{c}$} & \multicolumn{2}{c}{-} \\
& Strouhal & $\mathrm{PSD}_{\text {adim. }}$ & Strouhal & PSD $_{\text {adim. }}$ & Strouhal & PSD $_{\text {adim. }}$ \\
\cline { 2 - 7 } FG fijo & 0.090 & 14 & 0.095 & 156 & - & - \\
$\mathrm{k}_{1}$ & 0.023 & 79 & 0.025 & 301 & - & - \\
$\mathrm{k}_{2}$ & 0.033 & 583 & 0.031 & 270 & - & - \\
$\mathrm{k}_{3}$ & 0.038 & 608 & 0.037 & 260 & - & - \\
\hline
\end{tabular}

Tabla 5.5: Strouhal y PSD adimensional vs. $k$ (Mini-flap Rotante, $\mathrm{y} / \mathrm{c}=-2 \% \mathrm{c}$ ).

\begin{tabular}{ccccccc}
\hline & \multicolumn{2}{c}{$\mathrm{x} / \mathrm{c}=2 \% \mathrm{c}$} & \multicolumn{2}{c}{$\mathrm{x} / \mathrm{c}=4 \% \mathrm{c}$} & \multicolumn{2}{c}{$\mathrm{x} / \mathrm{c}=10 \% \mathrm{c}$} \\
& Strouhal & $\mathrm{PSD}_{\text {adim. }}$ & Strouhal & $\mathrm{PSD}_{\text {adim. }}$ & Strouhal & PSD $_{\text {adim. }}$ \\
\cline { 2 - 7 } FG fijo & 0.140 & 34 & 0.137 & 152 & 0.114 & 59 \\
$\mathrm{k}_{1}$ & 0.016 & 1600 & 0.018 & 105 & 0.014 & 730 \\
$\mathrm{k}_{2}$ & 0.036 & 625 & 0.037 & 218 & 0.029 & 23 \\
$\mathrm{k}_{3}$ & 0.041 & 578 & 0.040 & 184 & 0.034 & 52 \\
\hline
\end{tabular}

Tabla 5.6: Strouhal y PSD adimensional vs. $k$ (Mini-flap Rotante $30^{\circ}, \mathrm{y} / \mathrm{c}=-2 \% \mathrm{c}$ ).

\begin{tabular}{ccccccc}
\hline & \multicolumn{2}{c}{$\mathrm{x} / \mathrm{c}=2 \% \mathrm{c}$} & \multicolumn{2}{c}{$\mathrm{x} / \mathrm{c}=4 \% \mathrm{c}$} & \multicolumn{2}{c}{$\mathrm{x} / \mathrm{c}=10 \% \mathrm{c}$} \\
& Strouhal & $\mathrm{PSD}_{\text {adim. }}$ & Strouhal & $\mathrm{PSD}_{\text {adim. }}$ & Strouhal & PSD $_{\text {adim. }}$ \\
\cline { 2 - 7 } FG fijo & 0.080 & 16 & 0.072 & 20 & 0.078 & 88 \\
$\mathrm{k}_{1}$ & 0.018 & 352 & 0.017 & 318 & 0.016 & 206 \\
$\mathrm{k}_{2}$ & 0.031 & 319 & 0.031 & 486 & 0.033 & 601 \\
$\mathrm{k}_{3}$ & 0.035 & 555 & 0.034 & 490 & 0.035 & 821 \\
\hline
\end{tabular}

Tabla 5.7: Strouhal y PSD adimensional vs. $k$ (Mini-flap Móvil, y/c $=-2 \%$ c).

Se puede observar en las tablas que en la mayoría de los casos hay buena correlación entre las frecuencias de activación de los mecanismos y las frecuencias detectadas mediante el análisis del espectro de densidad de potencia detrás del borde de fuga del perfil a diferentes alturas. Esto nos hace pensar que el desarrollo de las estructuras turbulentas se ve forzado a intervalos de tiempo similares a los intervalos de actuación de los dispositivos de control actuante y no como lo haría de forma natural si el mini-flap Gurney estuviese fijo.

Si se analizan los diferentes casos ya sea a diferentes alturas y posiciones aguas abajo, no se observa una variación importante en el número de Strouhal. Este comportamiento era esperado ya que como se explicó anteriormente el desarrollo de las 
estructuras detrás del borde de fuga es de la forma de una calle de vórtices de von Kármán asimétrica por lo que la convección forzada de vórtices es periódica.

Analizando el mini-flap Gurney rotante, se observa que en los casos con frecuencias más altas tienen mayor energía que los casos de baja frecuencia y con mini-flap fijo. Si nos movemos aguas abajo $(75 \%$ c) el comportamiento se invierte, es decir, los que tenían menor intensidad se amplifican. Para el caso del mini-flap rotante (variante $30^{\circ}$ ), si bien no hay mediciones al $75 \%$ c, el comportamiento tiende a ser similar al primer caso. En el mini-flap móvil es más acentuado el comportamiento en el caso del mini-flap fijo donde las intensidades se ven amplificadas mientras que el resto de los casos se mantienen constantes. En este caso tampoco se dispone de mediciones al $75 \%$ c.

Por lo tanto la tendencia generalizada a todos los dispositivos es que para la frecuencia de activación 1 los picos se amplifican aguas abajo, mientras que para el resto de las frecuencias disminuyen su intensidad. Para el caso del mini-flap fijo en algunos mecanismos la intensidad se mantiene aproximadamente constante o tiene un comportamiento similar a la frecuencia 1. En los casos donde se midió hasta el 10\%c detrás del borde de fuga es difícil establecer una tendencia respecto del comportamiento de las intensidades de energía aguas abajo ya que a esta posición detrás del borde de fuga estamos todavía en la estela cercana.

Por otro lado, la disminución en la intensidad de los picos de potencia aguas abajo se puede deber a la pérdida de energía por efectos viscosos para escalas más pequeñas $[25$, $27,28]$. 


\section{CAPÍtulo 6}

\section{CONCLUSIONES}

\subsection{Evaluación de Resultados}

Los trabajos de investigación presentados en esta tesis se centraron en la implementación de dispositivos de control de flujo tanto pasivos como activos en un perfil aerodinámico, como así también en el análisis de la estela cercana detrás del borde de fuga mediante la técnica de anemometría de hilo caliente, lo que nos permitirá deducir el grado de alcance de los objetivos mencionados en los aspectos introductorios de esta tesis.

Dichos mecanismos están basados en la idea del mini-flap Gurney y cuentan con una placa plana de una altura no mayor al $2 \%$ c dispuestos a lo largo de la envergadura del modelo. Se pensó en dos alternativas a la hora de activar los dispositivos logrando movimientos de rotación y traslación según el modelo estudiado.

Se ha encontrado buena concordancia entre los trabajos presentes en la bibliografía y los resultados obtenidos en el presente estudio de los cuales se hará referencia a continuación.

Como ya se mencionó anteriormente, no es correcto estimar la resistencia del perfil utilizando el perfil de velocidades medias en la estela cercana ya que en el procedimiento normal se mide dicho perfil a una cuerda por detrás del borde de fuga. En este estudio se decidió establecer las tendencias de la resistencia aerodinámica en forma cualitativa en base al desarrollo de los perfiles de velocidad tomando mediciones en diferentes estaciones aguas abajo.

Teniendo en cuenta esto y como era de esperar podemos decir que el mini-flap Gurney fijo incrementa la resistencia total aerodinámica respecto del perfil solo [25, 26, 28, 35]. Del análisis de las gráficas de velocidad medias se desprende que dicho dispositivo pasivo no solo reduce la cantidad de movimiento del fluido detrás del borde de fuga sino que también esta reducción se da en un rango mayor de alturas incrementando aún más la perdida de cantidad de movimiento. Comparando los dispositivos de actuación en forma completamente desplegada el mini-flap Gurney fijo dispuesto a $90^{\circ}$ (caso móvil) produce mayores pérdidas en la velocidad respecto del mini-flap dispuesto a $30^{\circ}$ (caso rotante). Este comportamiento 
fue analizado por otros autores donde se estudió la influencia del ángulo de montaje del mini-flap sobre los coeficientes aerodinámicos $\left(C_{L}\right.$ y $\left.C_{D}\right)$ [36].

Asimismo, se corrobora que el mini-flap Gurney fijo produce un aumento en la circulación del perfil ya que en los diagramas de vectores de velocidad media se observa como este dispositivo incrementa el módulo de los vectores de velocidad con fluido proveniente del extradós mientras que los módulos de los vectores del intradós se ven reducidos $[25,32,35]$. El comportamiento del perfil solo es opuesto con lo que se verifica que efectivamente el mini-flap Gurney fijo incrementa la circulación media del perfil teniendo en cuenta el concepto de circulación como la integral de las velocidades alrededor del cuerpo aerodinámico. Esta es una presunción ya que no existe un cálculo directo de la circulación si no que se analizan solo un conjunto de vectores en la estela cercana. Esto se podría verificar mediante la medición de cargas aerodinámicas en el perfil.

Existe una relación entre la frecuencia de accionamiento $F$ y el tamaño de las escalas $L_{I}$. El hecho de que frecuencias más bajas producen escalas más grandes puede explicarse asumiendo que el tiempo de actividad (tiempo desplegado y retraído) de dichos dispositivos es inversamente proporcional a la frecuencia y que los mecanismos están diseñados de forma que para una revolución de motor el mini-flap se encuentra solamente un instante de tiempo completamente desplegado y otro instante completamente retraído. Teniendo en cuenta que el desarrollo de las estructuras se produce por convección forzada aguas abajo como consecuencia de la incidencia de la corriente de aire en la zona circundante al mini-flap Gurney al aumentar la frecuencia este instante de tiempo se ve reducido junto con la escala de tiempo de dicha estructura dando como resultado estructuras más pequeñas.

Una comparación interesante resulta del análisis de los vectores de velocidad media y las escalas espaciales para los diferentes casos y posiciones. Como ya dijimos las escalas de la componente $u$ se agrandan mientras que las escalas de la componente $v$ se achican a medida que nos movemos aguas abajo. Al mismo tiempo los vectores de velocidad se ven modificados en la misma forma, es decir la componente $u$ tiene mayor intensidad mientras que la componente $v$ se reduce. De allí se puede deducir que el tamaño de las escalas espaciales de las estructuras influye sobre el campo de vectores en la estela. Se observó por ejemplo que escalas más grandes en la componente $v$ produce variaciones más importantes de la velocidad en la misma dirección. En el caso del mini-flap rotante (variante $30^{\circ}$ ) las escalas espaciales más grandes en $v$ se dan para la frecuencia 1 pero aun así las componentes de velocidad verticales son mayores en el caso del mini-flap Gurney fijo. Si tenemos en cuenta la energía de las estructuras analizando los gráficos de $P S D$ vemos que el caso del mini-flap fijo tiene mayor intensidad por lo que podría explicar el aumento de downwash respecto a los otros casos. Este efecto se observa también en el caso del mini-flap Gurney móvil donde el tamaño de las escalas verticales es mucho más grande que el resto de los casos aun con menores intensidades viéndose reflejado en el diagrama de vectores con un aumento en la componente descendente. Por lo tanto podemos afirmar que el tamaño de las escalas espaciales en $v$ junto con la energía de dichas estructuras son las que influyen en las componentes de velocidad vertical y por ende en la sustentación del perfil [25, 35]. 


\section{CAPÍTULO 6. CONCLUSIONES}

Si pensamos en los perfiles de velocidad media detrás del borde de fuga vemos que los mínimos de velocidad se ven alterados en altura respecto del borde de fuga cuando se modifica la frecuencia de activación. Si bien el dispositivo que produce mayor cambio es el min-flap Gurney fijo los cambios introducidos por las variantes de frecuencia se encuentran en un caso intermedio donde puedo modificar este punto variando la frecuencia. Si aumento la frecuencia entonces el punto se ubica más cerca del borde de fuga del perfil tendiendo al caso del perfil solo. Por lo tanto la implementación de dichos mecanismos produce una modificación en el punto de estagnación posterior de forma similar al cambio que produce el mini-flap Gurney fijo cambiando la curvatura virtual del perfil [25, 26, 35].

Analizando los casos con frecuencia en la estela cercana (hasta el 10\%c) se observó que la implementación de dispositivos activos de control de flujo producen menos perdida de cantidad de movimiento en la velocidad del fluido respecto de los casos con y sin mini-flap logrando una reducción en la resistencia aerodinámica. El hecho de modificar el tamaño y frecuencia de desprendimientos detrás del borde de fuga hace que el campo fluido-dinámico se vea alterado de forma tal de modificar la circulación del mismo reduciendo la resistencia del perfil. Como ya se dijo anteriormente, es una suposición que habría que corroborar por otros métodos como ser mediciones de carga o tomar mediciones de velocidad en la estela lejana.

El perfil solo es el que introduce menos intensidad de turbulencia en la estela como era de esperar ya que podríamos estar hablando de las escalas más pequeñas y no de las grandes fluctuaciones introducidas por los mecanismos. Detrás del borde de fuga se ve que estos dispositivos incrementan en gran medida la complejidad del campo fluido-dinámico haciendo el flujo más turbulento. Para los casos con frecuencia la mayor intensidad de turbulencia se da para la frecuencia 1 tanto en el caso rotante de $30^{\circ}$ como en el caso móvil con valores similares.

Haciendo un análisis en conjunto de los valores de intensidad de turbulencia y el tamaño de las escalas espaciales vemos que un incremento en la turbulencia es producto del incremento del tamaño de la escala. Este comportamiento se da en ambas componentes por lo que nos da una idea de que las escalas más grandes producen mayores fluctuaciones en las componentes de velocidad y vice-versa. Pensando en la definición de la intensidad de turbulencia según la ecuación 4.10, podemos decir que el hecho de disponer de estructuras con escalas más grandes produce mayores fluctuaciones en el tiempo en ambas componentes de velocidad dando como resultado un aumento en la intensidad de turbulencia. Las gráficas de intensidad de turbulencia corroboran este comportamiento con una tendencia definida respecto de la frecuencia de activación de los mecanismos: a mayor frecuencia entonces la intensidad de turbulencia disminuye [26]. 


\subsection{Conclusiones Generales}

El análisis de las gráficas nos permite concluir que existe un comportamiento generalizado respecto de los mecanismos de control de flujo. Es así que cuando dichos dispositivos son accionados modifican el comportamiento del fluido en la estela cercana y por ende las características aerodinámicas del perfil. Pero dicho comportamiento se ve limitado entre el caso del perfil solo y el caso del mini-flap Gurney fijo como elemento pasivo, es decir que con la actuación de los mecanismos podemos influenciar al fluido en las cercanías del borde de fuga de modo que se comportase tanto como la condición de "perfil solo" cuando se aplican frecuencias más altas como la condición de perfil con "mini-flap Gurney fijo" para frecuencias más bajas pasando por estados intermedios.

A continuación se detallan alguno de los efectos producidos cuando los dispositivos son accionados.

Aumentando la frecuencia de activación de los mecanismos:

- Disminuye la intensidad de turbulencia.

- Disminuye el downwash y la circulación del perfil.

- Disminuye el módulo de los vectores de velocidad media.

- Aumenta la frecuencia de desprendimiento de las estructuras turbulentas.

- Disminuye el tamaño de las escalas espaciales de las estructuras turbulentas.

- Desplaza el punto de estagnación posterior cerca del borde de fuga y por ende disminuye la curvatura media virtual del perfil.

Se observó claramente que la estela cercana de un perfil puede ser modificada y manipulada mediante dispositivos activos y pasivos de control de flujo con diferentes objetivos. Hemos encontrado estudiando la estela turbulenta detrás del perfil influenciada por los dispositivos, la relación entre las escalas temporales y espaciales, intensidades de turbulencias, frecuencias de desprendimiento y velocidades medias, entre otras. Este conjunto de variables que caracterizan el flujo de estela modifican la circulación sobre el perfil y por ende las fuerzas resultantes que actúan sobre el mismo como ser la sustentación y la resistencia aerodinámica.

Por lo tanto si es posible manipular estas variables mediante la implementación de dichos mecanismos podemos asumir entonces que se puede lograr el control del fluido en la cercanía del borde fuga con beneficios tales como el retraso de perdida en perfiles, modificación de la carga aerodinámica para evitar roturas por fatiga debido a ráfagas de viento en palas de generadores eólicos, simplificación de superficies de control en vehículos no tripulados, aumento de la eficiencia en vuelo crucero o mejora de las condiciones de sustentación incrementando la seguridad de las fases de despegue y aterrizaje en aviones de gran porte, entre otros. 


\subsection{Futuras Líneas de Investigación}

A partir de los estudios realizados en las diferentes configuraciones, la continuación al trabajo presentado consiste en establecer la relación entre el patrón fluido-dinámico en la estela cercana y los coeficientes aerodinámicos $\left(C_{L}\right.$ y $\left.C_{D}\right)$. En este sentido, las tareas estarán dirigidas a realizar ensayos de mediciones de cargas aerodinámicas como la sustentación y la resistencia del perfil en correlación con mediciones de anemometría de hilo caliente en la estela. Dichos experimentos nos conducirán a cuantificar las fluctuaciones de la circulación del perfil cuando se generan diferentes estructuras turbulentas actuando dispositivos de control de flujo como el mini-flap Gurney. En particular, nos permitirá estudiar la influencia de la asimetría de la calle de vórtices sobre la circulación y que tipo de estructuras colaboran a la sustentación y cuales la reducen [27, 28].

Es sabido que existe una limitación técnica a la hora de determinar valores de velocidad en todo el campo del fluido al mismo tiempo mediante la técnica de anemometría de hilo caliente. Es por ello que los nuevos trabajos nos permitirían establecer nuevos puntos de medición en la estela a modo de poder determinar un campo fluido-dinámico más discreto y entonces poder comprender de forma más eficiente el comportamiento del fluido. Este tipo de determinación podría complementarse utilizando técnicas avanzadas de medición como PIV (Particle Image Velocimetry). Con esta técnica sería posible estudiar el tamaño de las escalas y la energía de dichos vórtices en un campo fluido-dinámico más discreto que con la técnica de anemometría lo cual nos daría una visión más realista de la calle asimétrica de vórtices en la estela cercana.

Finalmente, sería interesante estudiar nuevos valores de frecuencias de actuación dentro de las limitaciones físicas de los mecanismos tanto en el rango de bajas frecuencias como frecuencias superiores a las utilizadas en el presente trabajo. Para ello los trabajos futuros estarían enfocados también al desarrollo de nuevos modelos con dispositivos de prestaciones superiores a los actuales. 


\section{APÉNDICE A}

\section{Características de Perfil NACA 4412}

\begin{tabular}{ccc}
\hline $\mathrm{x} / \mathrm{c}$ & $\mathrm{y} / \mathrm{c}_{\text {extradós }}$ & $\mathrm{y} / \mathrm{c}_{\text {intradós }}$ \\
\hline 0.0000 & 0.0000 & 0.0000 \\
0.0125 & 0.0244 & -0.0143 \\
0.0250 & 0.0339 & -0.0195 \\
0.0500 & 0.0473 & -0.0249 \\
0.0750 & 0.0576 & -0.0274 \\
0.1000 & 0.0659 & -0.0286 \\
0.1500 & 0.0789 & -0.0288 \\
0.2000 & 0.0880 & -0.0274 \\
0.2500 & 0.0941 & -0.0250 \\
0.3000 & 0.0976 & -0.0226 \\
0.4000 & 0.0980 & -0.0180 \\
0.5000 & 0.0919 & -0.0140 \\
0.6000 & 0.0814 & -0.0100 \\
0.7000 & 0.0669 & -0.0065 \\
0.8000 & 0.0489 & -0.0039 \\
0.9000 & 0.0271 & -0.0022 \\
0.9500 & 0.0147 & -0.0016 \\
1.0000 & 0.0000 & 0.0000 \\
\hline
\end{tabular}

Tabla A.1: Coordenadas de perfil NACA 4412. 


\begin{tabular}{llll}
\hline Característica & Valor & Característica & Valor \\
\hline Espesor & $12.0 \% \mathrm{c}$ & $C_{L}$ máximo & 1.507 \\
Curvatura & $4.0 \% \mathrm{c}$ & Ángulo máximo $C_{L}$ & $10.0^{\circ}$ \\
Ángulo de borde de fuga & $14.4^{\circ}$ & Eficiencia máxima & 57.209 \\
Planicie inferior & $76.1 \% \mathrm{c}$ & Ángulo Eficiencia máx. & $5.5^{\circ}$ \\
Radio borde de ataque & $1.7 \% \mathrm{c}$ & $C_{L}$ Eficiencia máx. & 1.188 \\
Ángulo de pérdida & $11.0^{\circ}$ & Ángulo $C_{L}$ & $-4.0^{\circ}$ \\
\hline
\end{tabular}

Tabla A.2: Características geométricas y aerodinámicas del perfil NACA 4412 (Re 500000). 


\section{APÉNDICE B}

\section{ESPECIFICACIONES TÉCNICAS DE INSTRUMENTAL}

\begin{tabular}{ll}
\hline Característica & Valor \\
\hline Marca & DANTEC \\
Tipo & $55 R 51$ \\
Modelo & Fiber film sensor \\
Material & Níquel \\
Espesor & $0.5 \mu \mathrm{m}$ \\
Resistencia a $20^{\circ} \mathrm{C}$ & 3.7 (sensor 1), 5.3 (sensor 2) \\
Resistencia cable & 6.5 (sensor 1), 0.5 (sensor 2) \\
Coeficiente de temperatura de & \\
resistencia (TCR) & $0.52 \%$ (sensor 1), 0.46\% (sensor 2) \\
Temperatura máxima del sensor & $300^{\circ} \mathrm{C}$ \\
Temperatura ambiente máxima & $150^{\circ} \mathrm{C}$ \\
Velocidad máxima & $350 \mathrm{~m} / \mathrm{s}$ \\
Velocidad mínima & $0.2 \mathrm{~m} / \mathrm{s}$ \\
Frecuencia límite & $175 \mathrm{KHz}$ \\
\hline
\end{tabular}

Tabla B.1: Características técnicas de sensor de velocidad del viento. 


\begin{tabular}{ll}
\hline Característica & Valor \\
\hline Marca & DANTEC \\
Modelo & Streamline 90N10 (Frame) \\
& Streamline 90C10 (Módulos) \\
Número de módulos & 6 \\
Rango de temperaturas & 0 a $150^{\circ} \mathrm{C}\left(+/-0.5^{\circ} \mathrm{C}\right)$ \\
Frecuencia máxima de muestreo & $20 \mathrm{MHz}$ \\
Salida de voltaje & $0 \mathrm{a} 10 \mathrm{Volts}$ \\
\hline
\end{tabular}

Tabla B.2: Características técnicas de anemómetro de hilo caliente.

\begin{tabular}{lll}
\hline Característica & \multicolumn{1}{l}{ Valor } & \\
\hline Marca & DANTEC & \\
Modelo & Flowmaster & $54 \mathrm{~N} 60$ \\
Resolución de velocidad & $0.001 \mathrm{~m} / \mathrm{s}$ & $($ rango 0.1 a $0.999 \mathrm{~m} / \mathrm{s})$ \\
& $0.01 \mathrm{~m} / \mathrm{s}$ & $($ rango 1 a $9.99 \mathrm{~m} / \mathrm{s})$ \\
& $0.1 \mathrm{~m} / \mathrm{s}$ & $($ rango 10 a $30 \mathrm{~m} / \mathrm{s})$ \\
& $+/-2 \%$ & $($ rango 0.1 a $0.999 \mathrm{~m} / \mathrm{s})$ \\
& $+/-2.5 \%$ & $($ rango 1 a $9.99 \mathrm{~m} / \mathrm{s})$ \\
Precisión velocidad & $+/-2.5 \%$ & $($ rango 10 a $30 \mathrm{~m} / \mathrm{s})$ \\
& $+/-0.1^{\circ} \mathrm{C}$ & $\left(\right.$ rango $\left.-15 / 85^{\circ} \mathrm{C}\right)$ \\
& $+/-0.7^{\circ} \mathrm{C}$ & $\left(\right.$ rango 15 a $\left.0^{\circ} \mathrm{C}\right)$ \\
Resolución de temperatura & $+/-0.5^{\circ} \mathrm{C}$ & $\left(\right.$ rango 0 a $\left.40^{\circ} \mathrm{C}\right)$ \\
Precisión temperatura & $+/-0.7^{\circ} \mathrm{C}$ & $\left(\right.$ rango 40 a $\left.85^{\circ} \mathrm{C}\right)$ \\
& & \\
\hline
\end{tabular}

Tabla B.3: Características técnicas de termo-anemómetro de hilo caliente. 


\section{BIBLIOGRAFÍA}

[1] Hah, C. \& Lakshminarayana, B. Measurement and prediction of mean velocity and turbulence structure in the near wake of an airfoil. Journal of Fluid Mechanics, 115: 251-282 (1982).

[2] Liebeck, R.H. Design of subsonic airfoils for high lift. Journal of Aircraft, 15(9): 547561 (1978).

[3] Neuhart, D.H. \& Pendergraft, O.C. A water tunnel study of Gurney flaps. NASA TM-4071, Scientific and Technical Information Division (1988).

[4] Bloy, A.W. \& Durrant, M.T. Aerodynamic Characteristics of an aerofoil with Small Trailing Edge Flaps. Wind Engineering, 19(3): 167-172 (1995).

[5] Storms, B.L. \& Jang, C.S. Lift Enhancement of an Airfoil Using a Gurney Flap and Vortex Generators. Journal of Aircraft, 31(3): 542-547 (1994).

[6] Giguére, P., Lemay, J. \& Dumas, G. Gurney Flap Effects and Scaling for Low-Speed Airfoils. AIAA Paper 95-1881, 13th AIAA Applied Aerodynamics Conference (1995).

[7] Jeffery, D.R.M. \& Hurst, D.W. Aerodynamics of the Gurney Flap. AIAA Applied Aerodynamic Conference, AIAA 96-2418-CP (1996).

[8] Jeffrey, D.R.M., Hurst D.W., \& Zhang, X. Aerodynamics of Gurney Flaps on a Single-Element High-Lift Wing. Journal of Aircraft, 37(2): 295-301 (2000).

[9] Bacchi, F., Marañón Di Leo, J., Delnero, J.S., Colman, J., Martinez, M., Camocardi, M. \& Boldes, U. Determinación experimental del efecto de mini flaps Gurney sobre un perfil HQ-17. IX Reunión Sobre Recientes Avances En Física de Fluidos y sus Aplicaciones (2006).

[10] Dam, C. P. Van, Vijgen, P.M.H.W. \& Yen, D.T. Gurney Flap Experiments on Airfoil and Wings. Journal of Aircraft, 36(2): 484-486 (1999).

[11] Bechert, D.W., Meyer, R. \& Hage, W. Drag Reduction of Airfoils with Miniflaps. Can We Learn From Dragonflies? AIAA-2000-2315 (2000).

[12] Schatz, M., Guenther, B. \& Thiele, F. Computational Modeling of the Unsteady Wake behind Gurney-Flaps. $2^{\text {nd }}$ AIAA Flow Control Conference, AIAA-2417 (2004). 
[13] Huerre, P. \& Monkewitz, P. Absolute and convective instabilities in free shear layers. Journal of Fluid Mechanics, 159: 151-168 (1985).

[14] Kuethe, A.M. \& Schetzer, J.D. Foundations of Aerodynamics. John Wiley \& Sons, Inc., New York, ISBN 0471509523 (1959).

[15] Fiedler, H. E. Control of Free Turbulent Shear Flows. En Flow Control: Fundamentals and Practices (ed. Gad-el-Hak, M., Pollard, A., Bonnet, J. P.), 335429 (1998).

[16] Gad-el-Hak, M. Flow Control Passive, Active, and Reactive Flow Management. Cambridge: Cambridge UP (2000).

[17] Barlow, J.B., Rae, W.H. \& Pope, A. Low-speed Wind Tunnel Testing. New York: Wiley (1999).

[18] Scarabino, A. Características de la turbulencia atmosférica en un bosque de coníferas. Tesis Doctoral, Facultad de Ingeniería, UNLP (2005).

[19] Bruun, H.H. Hot Wire Anemometry. Oxford University Press (1995).

[20] Kaimal J.C. \& Finnigan, J.J. Atmospheric Boundary Layer Flows. Oxford University Press (1994).

[21] Castro, I.P. An Introduction to the Digital Analysis of Stationary Signals. Adam Hilger, Bristol (1989).

[22] Panofsky, H. \& Dutton, J.A. Atmospheric Turbulence. Models and Methods for Engineering Applications. John Wiley \& Sons, New York (1984).

[23] Jenkins, J.M. \& Watts, D.G. Spectral Analysis and its Applications. Holden Day, San Franciso (1968).

[24] Schwartz, M. \& Shaw, L. Signal Processing. Mc Graw Hill (1975).

[25] Boldes, U., Delnero, J.S., Marañon Di Leo, J., Colman, J. \& Camocardi, M. The wake asymmetry of an airfoil with a Gurney flap and their connection with the observed lift increase. International Review of Aerospace Engineering, ISBN 19737459 (IREASE), 3(2): 89-95 (2010).

[26] Delnero, J.S., Marañón Di Leo, J., Colman, J., García Sainz, M., Muñoz, F., Hérouard, N. \& Camocardi, M.E. Aspects of the influence of an oscillating mini-flap upon the near wake of an airfoil NACA 4412. Journal of Physics Conference Series (JPCS). Institute of Physics Publishing, UK. Conference Series 296012007 (2011).

[27] Camocardi, M., Delnero, J.S., Marañón Di Leo, J., Colman, J., Muñoz, F. \& García Sainz, M. Aspectos del control de flujo con un flap Gurney rotante sobre un perfil NACA 4412. Primeras Jornadas de Investigación y Transferencia UNLP, La Plata, Argentina (2011). 
[28] Camocardi, M., Marañon Di Leo, J., Delnero, J.S. \& Colman, J. Experimental study of a NACA 4412 airfoil with movable Gurney flap. $49^{\text {th }}$ AIAA Aerospace Sciences Meeting and Exhibit, Orlando (2011).

[29] Boldes, U., Delnero, J.S., Marañón Di Leo, J., Colman, J., Camocardi, M. \& François, D. Influencia en la sustentación, de los vórtices de la estela de un perfil con mini-flap tipo Gurney. Primer Congreso Argentino de Ingeniería Aeronáutica. La Plata, Argentina (2008).

[30] Camocardi, M., Delnero, J.S., Marañon Di Leo, J., Colman, J. \& Muñoz, F. Estudio experimental de un perfil NACA 4412 con flap Gurney rotante. Segundo Congreso de Ingeniería Aeronáutica. Córdoba, Argentina (2010).

[31] Camocardi, M.E., Delnero, J.S., Marañon Di Leo, J., Colman, J. \& Martinez, M.A. Analysis of a Gurney flap as an active and passive system. European Drag Reduction and Flow Control Meeting, Kyiv, Ukraine (2010).

[32] Bacchi, F., Marañón Di Leo, J., Delnero, J.S., Colman, J., Martinez, M., Camocardi M. \& Boldes, U. Determinación experimental del efecto de mini-flaps Gurney sobre un perfil HQ-17. IX Reunión sobre Recientes Avances en Física de Fluidos y sus Aplicaciones, Mendoza, Argentina (2006).

[33] Delnero, J.S., Marañón Di Leo, J., Colman, J., García Sainz, M., Muñoz, F., Hérouard, N. \& Camocardi, M. Aspectos de la influencia de un mini-flap oscilante en la estela cercana de un perfil NACA 4412. XI Reunión sobre Recientes Avances en Física de Fluidos y sus Aplicaciones, Colonia del Sacramento, Uruguay (2010).

[34] Delnero, J.S., Marañon Di Leo, J., Camocardi, M., François, D. \& Colman, J. Turbulent flow experiments of Gurney mini-flaps in low Reynolds number airfoil. Tenth J.J. Giambiagi Winter School: Principles and Applications of Fluid Dynamics, Buenos Aires, Argentina (2008).

[35] Delnero, J.S., Marañon Di Leo, J., Colman, J., García Sainz, M., Muñoz, F., \& Camocardi, M. About the effects of an oscillating mini-flap upon the wake on an airfoil, all inmersed in turbulent flow. Journal of Physics: Conference Series 318 062005 (ISSN: 1742-6596) (2011).

[36] Wang, J., Li, Y. \& Choi, K. Gurney flap-Lift enhancement, mechanisms and applications. Progress in Aerospace Sciences, 44(1): 22-47 (2007).

[37] Delnero, J.S. Comportamiento aerodinámico de perfiles de bajo Reynolds, inmersos en flujo turbulento. Tesis Doctoral, Facultad de Ingeniería, UNLP (2007).

[38] Wang, J., Zhan, J., Zhang, W. \& Wu, Z. Application of a Gurney Mini-flap on a Simplified Forward-Swept Aircraft Model. Beijing University of Aeronautics and Astronautics. Journal of Aircraft. 43(5) (2006). 
[39] Patel, M.P., Kolacinski, R.M., Patil, S.K.R. \& Terry T. Distributed Mechanical Actuators for Design of a Closed-loop Flow-control System. 3rd AIAA Flow Control Conference (2006).

[40] Mayda, E.A.; van Dam, C.P. \& Yen Nakafuji, D. Computational investigation of finite width microtabs for aerodynamic load control. AIAA-2005-1185 (2005).

[41] van Dam, C.P.; Chow, R.; Zayas, J.R. \& Berg, D.E. Computational investigations of small deploying tabs and mini-flaps for aerodynamic load control. Journal of Physics: Conference Series 75 012027 (2007).

[42] Matalanis, C.G. \& Eaton, J.K. Wake vortex alleviation using rapidly actuated segmented Gurney mini-flaps. Report 102. Flow Physics and Computation Division, Department of Mechanical Engineering, University of Stanford (2007).

[43] Bloy, A.W., \& Durrant, M.T. Aerodynamic Characteristics of an Aerofoil with Small Trailing Edge Mini-flaps. Wind Engineering. 19(3):167-172 (1995).

[44] Myose, R., Papadakis, M. \& Heron, I. Gurney Mini-flap Experiments on Airfoils, Wings, and Reflection Plane Model. Journal of Aircraft. 35(2):206-211 (1998).

[45] van Dam, C.P., Yen, D.T. \& Vijgen, P.M.H.W. Gurney Mini-flap Experiments on Airfoil and Wings. Journal of Aircraft. 36(2):484-486 (1999).

[46] Gai, S.L., \& Palfrey, R. Influence of Trailing-Edge Flow Control on Airfoil Performance. Journal of Aircraft. 40(2):332-337 (2003).

[47] Boldes, U., Delnero, J., Marañón Di Leo, J., Colman, J., Camocardi, M. \& François, D. Influencia en la sustentación, de los vórtices de la estela de un perfil con mini-flap tipo Gurney. 1er Congreso Nacional de Ingeniería Aeronáutica (2008).

[48] Tang, D. \& Dowel, E.H. Aerodynamic loading for an airfoil with an oscillating Gurney mini-flap. Journal of Aircraft. 44(4):1245-57 (2007).

[49] Hinze J. O. Turbulence. Ed. Mc Graw-Hill (1975).

[50] Hoerner S.F. Fluid - Dynamics Lift. Published by the Author (1975).

[51] Hoerner S.F. Fluid - Dynamics Drag. Published by the Author (1958).

[52] Gad-el-Hack, M. Modern Developments in Flow Control. Applied Mechanics Review 49:365 - 379 (1996).

[53] Ho, C.M. \& Huerre, P. Perturbed free shear layers. Annual Review of Fluid Mechanics. 16:365-424 (1984).

[54] Selig, M.S. The design of airfoils at low Reynolds numbers. AIAA-1985-74 American Institute of Aeronautics and Astronautics, Aerospace Sciences Meeting (1985). 
[55] Althaus, D. Recent Wind Tunnel Experiments at Low Reynolds Number. Aerodynamics at Low Reynolds Numbers $104<$ Re $<106$ International Conference. 2:18.1-18.42 (1986).

[56] Lachmann, G.V. Boundary Layer and Flow Control. Pergamon Press, Oxford, Gran Bretaña (1961).

[57] Gad-el-Hack, M. Interactive Control of Turbulent Boundary Layers: A Futuristic Overview. AIAA Journal. 32:1753-1765 (1994).

[58] Wassen E., Günther, B. \& Thiele, F. (Institute of Fluid Mechanics and Engineering Acoustics Technical University Berlin Germany) Delnero, J.S., Marañon Di Leo, J., Boldes, U., Colman, J., Bacchi, F. \& Martínez, M.A. (Dto. Aeronáutica Fac. Ingeniería UNLP Argentina) A Combined Numerical and Experimental Study of Mini-Flaps at Varying Positions on an Airfoils. 45th AIAA Aerospace Sciences Meeting and Exhibit (2007).

[59] Jänker, P., Grohmann, B., Lorkowski, T., Hermle, F., Friedl, S. \& Maucher, C. Smart Actuation Systems for Active Flow Control. First Active Flow Control Conference (2006).

[60] Petz, R. \& Wolfgang, N. Designing Actuators for Active Separation Control Experiments on High-Lift Configurations. First Active Flow Control Conference (2006).

[61] Giguère, P., Dumas, G. \& Lemay, J. Gurney Flap Scaling for Optimum Lift-to-Drag Ratio. AIAA Journal. 35(12):1888-1890 (1997).

[62] Greenblatt, D., Vey, S., Paschereit, O. \& Meyer, R. Flap Vortex Management Using Active Gurney Flaps. AIAA Journal. 47(12):2845-2856 (2009).

[63] Li, Y., Wang, J. \& Zhang, P. Influences of Mounting Angles and Locations on the Effects of Gurney Flaps. Journal of Aircraft. 40(3):494-498 (2003).

[64] Maughmer, M.D. \& Bramesfeld, G. Experimental Investigation of Gurney Flaps. Journal of Aircraft. 45(6):2062-2067 (2008).

[65] Nikolic, V. Additional Aerodynamic Features of Wing-Gurney Flap Flows. Journal of Aircraft. 43(5):1325-1333 (2006).

[66] Traub, L.W. Effects of Gurney Flaps on an Annular Wing. Journal of Aircraft 46(3):1085-1088 (2009).

[67] Zerihan, J., \& Zhang, X. Aerodynamics of Gurney Flaps on a Wing in Ground Effect. AIAA Journal. 39(5):772-780 (2001).

[68] Jeffrey, D.R.M., Zhang, X. \& Hurst, D.W. Aerodynamics of Gurney flaps on a single-element high-lift wing. Journal of Aircraft. 37(2):295-301 (2000). 
[69] Troolin, D.R., Longmire, E.K., \& Lai, W.T. Time resolved PIV analysis of flow over a NACA 0015 airfoil with Gurney flap. Experiments in Fluids. 41:241-254 (2006).

[70] Solovitz, S. \& Eaton, J. Spanwise Response Variation for Partial-Span Gurney-Type Flaps. AIAA Journal. 42(8):1640-1643 (2004).

[71] Solovitz, S. \& Eaton, J. Dynamic Flow Response Due to Motion of Partial-Span Gurney-Type Flaps. AIAA Journal. 42(9):1729-1736 (2004).

[72] Kentfield, J.A.C. The Potential of Gurney Flaps for Improving the Aerodynamic Performance of Helicopter Rotors. AIAA International Powered Lift Conference. 283-292 (1993).

[73] Meyer, R., Hage, W., Bechert, D.W., Schatz, M., \& Thiele, F. Drag Reduction on Gurney Flaps by Three-Dimensional Modifications. Journal of Aircraft. 43(1):132140 (2006).

[74] Traub, L.W. \& Agarwal, G. Aerodynamic Characteristics of a Gurney/Jet Flap at Low Reynolds Numbers. Journal of Aircraft. 45(2):424-429 (2008).

[75] Shea, P.R. \& Smith, D.R. Aerodynamic Control of a Rectangular Wing Using Gurney Flaps and Synthetic Jets. AIAA 2009-886 (2009).

[76] Zhang, P.F., Liu, A.B. \& Wang, J.J. Aerodynamic Modification of a NACA 0012 Airfoil by Trailing-Edge Plasma Gurney Flap. AIAA Journal. 47 (2009).

[77] Lee, T. Aerodynamic Characteristic of Airfoil with Perforated Gurney-Type Flaps. Journal of Aircraft. 46(2):442-448 (2009).

[78] Myose, R., Papadakis, M. \& Heron, I. Gurney Flap Experiments on Airfoils, Wings, and Reflection Plane Model. Journal of Aircraft. 35(2):206-211 (1998).

[79] Matalanis, C.G. \& Eaton, J.E. Wake Vortex Control Using Static Segmented Gurney Flaps. AIAA Journal. 45(2):321-328 (2007).

[80] Nikolic, V.R. Effect of Full-Span Gurney Flap Height on Wing Wake Vortex Alleviation. Journal of Aircraft. 43(5):1555-1558 (2006).

[81] Papadakis, M., Myose, R. Y., Heron, I., \& Johnson, B. L. An Experimental Investigation of Gurney Flaps on a GA(W)-2 Airfoil with $25 \%$ Slotted Flap. AIAA Paper 96-2437 (1996).

[82] Traub, L.W., Miller, A., \& Rediniotis, O. Preliminary Parametric Study of GurneyFlap Dependencies. Journal of Aircraft. 43(4):1242-1244 (2006).

[83] Myose, R., Heron, I., \& Papadakis, M. Effects of Gurney Flaps on a NACA 0011 Airfoil. AIAA Paper 96-0059 (1996).

[84] Traub, L.W., Miller, A., \& Rediniotis, O. Comparisons of a Gurney and Jet-Flap for Hinge-Less Control. Journal of Aircraft. 41(2):420-423 (2004). 